\title{
Refined analytic torsion as an element of the determinant line
}

\author{
MAXIM BRAVERMAN \\ THOMAS KAPPELER
}

\begin{abstract}
We construct a canonical element, called the refined analytic torsion, of the determinant line of the cohomology of a closed oriented odd-dimensional manifold $M$ with coefficients in a flat complex vector bundle $E$. We compute the Ray-Singer norm of the refined analytic torsion. In particular, if there exists a flat Hermitian metric on $E$, we show that this norm is equal to 1 . We prove a duality theorem, establishing a relationship between the refined analytic torsions corresponding to a flat connection and its dual.
\end{abstract}

58J52; 58J28, 57R20

\section{Introduction}

The aim of this paper is to define the notion of the refined analytic torsion associated to an arbitrary vector bundle $E \rightarrow M$ over a closed oriented manifold of odd dimension $d=2 r-1$ and an arbitrary flat connection $\nabla$ on $E$. The refined analytic torsion $\rho_{\text {an }}=\rho_{\text {an }}(\nabla)$ is a canonically defined element of the determinant line $\operatorname{Det}\left(H^{\bullet}(M, E)\right)$ of the cohomology of the bundle $E$.

The Ray-Singer norm of the refined analytic torsion is equal to $e^{\pi \operatorname{Im} \eta}$, where $\eta$ is the $\eta$-invariant of the Atiyah-Patodi-Singer odd signature operator $\mathcal{B}$ associated to $\nabla$ and a Riemannian metric on $M$. In particular, if the connection $\nabla$ is Hermitian then $\eta$ is real and the Ray-Singer norm of $\rho_{\mathrm{an}}(\nabla)$ is equal to 1 . This property justifies calling $\rho_{\text {an }}(\nabla)$ the refined analytic torsion. Indeed, the Ray-Singer torsion can be viewed as an element of the determinant line $\operatorname{Det}\left(H^{\bullet}(M, E)\right)$ whose Ray-Singer norm is equal to one. Such an element is defined up to a multiplication by $t \in \mathbb{C}$ with $|t|=1$. The refined analytic torsion $\rho_{\mathrm{an}}(\nabla)$ is a canonical choice of such an element.

If the connection $\nabla$ is acyclic, that is, if the cohomology $H^{\bullet}(M, E)=0$, then $\operatorname{Det}\left(H^{\bullet}(M, E)\right)$ is canonically isomorphic to $\mathbb{C}$ and the refined analytic torsion is a complex number. Under the additional assumption that $\mathcal{B}$ is bijective, we introduced this number and studied it in $[9 ; 5]$. In this paper we drop this assumption and define the refined analytic torsion for arbitrary flat connections and compare it to the Ray-Singer 
torsion. Recall that in $[9 ; 5]$, we also computed the quotient of the refined analytic torsion and Turaev's refinement of the combinatorial torsion. In our subsequent paper [8], we extend this computation to arbitrary flat connections.

Having applications in topology in mind Dan Burghelea suggested constructing a holomorphic function on the space of acyclic connections, whose absolute value is (related to) the Ray-Singer torsion. Such functions were constructed by Burghelea and Haller in $[14 ; 12]$ and also in our papers $[9 ; 5]$. The results of the present paper fit nicely into this program. In fact, in [8], we show that the refined analytic torsion is a holomorphic section of the determinant line bundle over the space of flat connections (or, equivalently, over the space of representations of the fundamental group of $M$ ). Thus we extend the construction of $[9 ; 5]$ to general, not necessarily acyclic, connections.

Let us now briefly describe our construction of the refined analytic torsion.

\subsection{The refined torsion of a finite dimensional complex}

Let

$$
\left(C^{\bullet}, \partial\right): \quad 0 \rightarrow C^{0} \stackrel{\partial}{\longrightarrow} C^{1} \stackrel{\partial}{\longrightarrow} \cdots \stackrel{\partial}{\longrightarrow} C^{d} \rightarrow 0
$$

be a complex finite dimensional $\mathbb{C}$-vector spaces of odd length $d=2 r-1$. A chirality operator $\Gamma: C^{\bullet} \rightarrow C^{\bullet}$ is an involution such that $\Gamma\left(C^{j}\right)=C^{d-j}$, for all $j=0, \ldots, d$. As a first step towards our construction of the refined analytic torsion we introduce and study the refined torsion of the pair $\left(C^{\bullet}, \Gamma\right)$. Consider the determinant line

$$
\operatorname{Det}\left(C^{\bullet}\right):=\bigotimes_{j=0}^{d} \operatorname{Det}\left(C^{j}\right)^{(-1)^{j}},
$$

where $\operatorname{Det}\left(C^{j}\right)^{-1}:=\operatorname{Hom}\left(C^{j}, \mathbb{C}\right)$ denotes the dual of $C^{j}$. For an element $c_{j} \in$ $\operatorname{Det}\left(C^{j}\right)$ we denote by $c_{j}^{-1}$ the unique element in $\operatorname{Det}\left(C^{j}\right)^{-1}$ satisfying $c_{j}^{-1}\left(c_{j}\right)=1$. We also denote by $\Gamma c_{j} \in \operatorname{Det}\left(C^{d-j}\right)$ the image of $c_{j}$ under the map $\operatorname{Det}\left(C^{j}\right) \rightarrow$ $\operatorname{Det}\left(C^{d-j}\right)$ induced by $\Gamma: C^{j} \rightarrow C^{d-j}$.

For each $j=0, \ldots, r-1$, fix an element $c_{j} \in \operatorname{Det}\left(C^{j}\right)$ and consider the element

$$
\begin{aligned}
c_{\Gamma}:=(-1)^{\mathcal{R}\left(C^{\bullet}\right)} \cdot c_{0} \otimes c_{1}^{-1} \otimes \cdots \otimes & c_{r-1}^{(-1)^{r-1}} \otimes \\
& \left(\Gamma c_{r-1}\right)^{(-1)^{r}} \otimes\left(\Gamma c_{r-2}\right)^{(-1)^{r-1}} \otimes \cdots \otimes\left(\Gamma c_{0}\right)^{-1}
\end{aligned}
$$

of $\operatorname{Det}\left(C^{\bullet}\right)$, where $(-1)^{\mathcal{R}\left(C^{\bullet}\right)}$ is a normalization factor introduced in (4-1). It is easy to see that $c_{\Gamma}$ is independent of the choice of $c_{0}, \ldots, c_{r-1}$. 
Denote by $H^{\bullet}(\partial)$ the cohomology of the complex $\left(C^{\bullet}, \partial\right)$. In Section 2.4, we construct a sign refined version of the standard isomorphism $\operatorname{Det}\left(C^{\bullet}\right) \rightarrow \operatorname{Det}\left(H^{\bullet}(\partial)\right)$, cf Milnor [25]. Our isomorphism $\phi_{C^{\bullet}}: \operatorname{Det}\left(C^{\bullet}\right) \rightarrow \operatorname{Det}\left(H^{\bullet}(\partial)\right)$ is similar but not equal to the one considered by Turaev [35].

Definition 1.2 The refined torsion of the pair $\left(C^{\bullet}, \Gamma\right)$ is the element

$$
\rho_{\Gamma}=\rho_{C \cdot, \Gamma}:=\phi_{C} \bullet\left(c_{\Gamma}\right) \in \operatorname{Det}\left(H^{\bullet}(\partial)\right) .
$$

\subsection{Calculation of the refined torsion of a finite dimensional complex}

To compute the refined torsion we introduce the operator

$$
\mathcal{B}:=\Gamma \partial+\partial \Gamma \text {. }
$$

This operator is a finite dimensional analogue of the signature operator on an odddimensional manifold, see Atiyah [1, page 44], [2, page 405], Gilkey [19, pages 64-65], and Section 7 of this paper. For $j=0, \ldots, d$, define

$$
C_{+}^{j}:=\operatorname{Ker}(\partial \circ \Gamma) \cap C^{j}, \quad C_{-}^{j}:=\operatorname{Ker} \partial \cap C^{j}
$$

and set $C_{+}^{-1}=C_{-}^{d+1}=0$. Let $\mathcal{B}_{j}$ and $\mathcal{B}_{j}^{ \pm}$denote the restriction of $\mathcal{B}$ to $C^{j}$ and $C_{ \pm}^{j}$ respectively. Then, for each $j=0, \ldots, d$, one has

$$
\mathcal{B}_{j}^{+}=\Gamma \circ \partial: C_{+}^{j} \longrightarrow C_{+}^{d-j-1}, \mathcal{B}_{j}^{-}=\partial \circ \Gamma: C_{-}^{j} \longrightarrow C_{-}^{d-j+1} .
$$

Denote $C^{\text {even }}:=\bigoplus_{j \text { even }} C^{j}$ and $C_{ \pm}^{\text {even }}:=\bigoplus_{j \text { even }} C_{ \pm}^{j}$. Set

$$
\mathcal{B}_{\text {even }}:=\bigoplus_{j \text { even }} \mathcal{B}_{j}: C^{\text {even }} \rightarrow C^{\text {even }}, \quad \mathcal{B}_{\text {even }}^{ \pm}:=\bigoplus_{j \text { even }} \mathcal{B}_{j}^{ \pm}: C_{ \pm}^{\text {even }} \rightarrow C_{ \pm}^{\text {even }}
$$

and define $\mathcal{B}_{\text {odd }}, \mathcal{B}_{\text {odd }}^{ \pm}$similarly. As $\mathcal{B}_{\text {even }}=\Gamma \circ \mathcal{B}_{\text {odd }} \circ \Gamma$, it turns out that it suffices to study $\mathcal{B}_{\text {even }}$.

Suppose, first, that the signature operator $\mathcal{B}$ is bijective. Then, cf Lemma 5.2, the complex $\left(C^{\bullet}, \partial\right)$ is acyclic. Hence, $\operatorname{Det}\left(H^{\bullet}(\partial)\right)$ is canonically identified with $\mathbb{C}$ and the refined torsion $\rho_{\Gamma}$ can be viewed as a number in $\mathbb{C}$. In Proposition 5.6 we compute this number and show that

$$
\rho_{\Gamma}=\operatorname{Det}_{\mathrm{gr}}\left(\mathcal{B}_{\text {even }}\right)
$$

where the graded determinant $\operatorname{Det}_{\text {gr }}\left(\mathcal{B}_{\text {even }}\right)$ is defined by the formula

$$
\operatorname{Det}_{\mathrm{gr}}\left(\mathcal{B}_{\text {even }}\right) \stackrel{\text { Def }}{=} \operatorname{Det}\left(\mathcal{B}_{\text {even }}^{+}\right) / \operatorname{Det}\left(-\mathcal{B}_{\text {even }}^{-}\right)
$$


Note that in our definition of the graded determinant the quotient is the determinant of the negative of $\mathcal{B}_{\text {even }}^{-}$.

To compute the refined torsion in the case $\mathcal{B}$ is not bijective, note that $\mathcal{B}^{2}=(\Gamma \partial)^{2}+$ $(\partial \Gamma)^{2}$ maps $C^{j}(j=0, \ldots, d)$ into itself. For each $j=0, \ldots, d$ and an arbitrary interval $\mathcal{I}$, denote by $C_{\mathcal{I}}^{j} \subset C^{j}$ the linear span of the generalized eigenvectors of the restriction of $\mathcal{B}^{2}$ to $C^{j}$, corresponding to eigenvalues $\lambda$ with $|\lambda| \in \mathcal{I}$. Since both operators, $\Gamma$ and $\partial$, commute with $\mathcal{B}$ (and, hence, with $\mathcal{B}^{2}$ ), $\Gamma\left(C_{\mathcal{I}}^{j}\right) \subset C_{\mathcal{I}}^{d-j}$ and $\partial\left(C_{\mathcal{I}}^{j}\right) \subset C_{\mathcal{I}}^{j+1}$. Hence, we obtain a subcomplex $C_{\mathcal{I}}^{\bullet}$ of $C^{\bullet}$ and the restriction $\Gamma_{\mathcal{I}}$ of $\Gamma$ to $C_{\mathcal{I}}^{\bullet}$ is a chirality operator for $C_{\mathcal{I}}^{\bullet}$. We denote by $H_{\mathcal{I}}^{\bullet}(\partial)$ the cohomology of the complex $\left(C_{\mathcal{I}}^{\bullet}, \partial_{\mathcal{I}}\right)$.

Denote by $\partial_{\mathcal{I}}, \mathcal{B}_{\mathcal{I}}$, and $\mathcal{B}_{\mathcal{I}}^{\text {even }}$ the restrictions of $\partial, \mathcal{B}$, and $\mathcal{B}_{\text {even }}$ to $C_{\mathcal{I}}$. Then $B_{\mathcal{I}}=\Gamma_{\mathcal{I}} \partial_{\mathcal{I}}+\partial_{\mathcal{I}} \Gamma_{\mathcal{I}}$ and one easily shows (cf Lemma 5.8) that $\left(C_{\mathcal{I}}^{\bullet}, \partial_{\mathcal{I}}\right)$ is acyclic if $0 \notin \mathcal{I}$.

For each $\lambda \geq 0, C^{\bullet}=C_{[0, \lambda]}^{\bullet} \oplus C_{(\lambda, \infty)}^{\bullet}$ and $H_{(\lambda, \infty)}^{\bullet}(\partial)=0$ whereas $H_{[0, \lambda]}^{\bullet}(\partial) \simeq H^{\bullet}(\partial)$. Hence, there are canonical isomorphisms

$$
\Phi: \operatorname{Det}\left(H_{(\lambda, \infty)}^{\bullet}(\partial)\right) \longrightarrow \mathbb{C}, \quad \Psi: \operatorname{Det}\left(H_{[0, \lambda]}^{\bullet}(\partial)\right) \longrightarrow \operatorname{Det}\left(H^{\bullet}(\partial)\right) .
$$

In the sequel, we will write $t$ for $\Phi(t) \in \mathbb{C}$ and denote by $h$ also the element $\Psi(h) \in$ $\operatorname{Det}\left(H^{\bullet}(\partial)\right)$. Then, cf Proposition 5.10, for any $\lambda \geq 0$, the refined torsion can be computed to be

$$
\rho_{\Gamma}=\operatorname{Det}_{\mathrm{gr}}\left(\mathcal{B}_{(\lambda, \infty)}^{\mathrm{even}}\right) \cdot \rho_{\Gamma_{[0, \lambda]}} .
$$

In particular, the element $\operatorname{Det}_{\text {gr }}\left(\mathcal{B}_{(\lambda, \infty)}^{\text {even }}\right) \cdot \rho_{\Gamma_{[0, \lambda]}}$ is independent of $\lambda$. It is this property which allows us to define the refined analytic torsion.

\subsection{The canonical element in the determinant line of the cohomology of a flat vector bundle over a Riemannian manifold}

In the second part of this paper (Sections 6-11) we apply the notion of refined torsion to define and investigate the refined analytic torsion of the (twisted) de Rham complex. Let $E \rightarrow M$ be a complex vector bundle over a closed manifold of odd dimension $d=2 r-1$ and let $\nabla$ be a flat connection on $E$. Let $\Omega^{\bullet}(M, E)$ denote the de Rham complex of $E$-valued differential forms on $M$. For a given Riemannian metric $g^{M}$ on $M$ denote by

$$
\Gamma=\Gamma\left(g^{M}\right): \Omega^{\bullet}(M, E) \longrightarrow \Omega^{\bullet}(M, E)
$$


the chirality operator (cf Berline-Getzler-Vergne [3, Section 3]), defined in terms of the Hodge $*$-operator by the formula

$$
\Gamma \omega:=i^{r}(-1)^{\frac{k(k+1)}{2}} * \omega, \quad \omega \in \Omega^{k}(M, E) .
$$

The odd signature operator introduced by Atiyah, Patodi, and Singer $[1 ; 2]$ (see also Gilkey [19]) is the first order elliptic differential operator $\mathcal{B}: \Omega^{\bullet}(M, E) \rightarrow \Omega^{\bullet}(M, E)$, given by

$$
\mathcal{B} \stackrel{\text { Def }}{=} \Gamma \nabla+\nabla \Gamma .
$$

Notice, that $\mathcal{B}^{2}$ maps $\Omega^{j}(M, E)$ into itself for every $j=0, \ldots, d$.

For an interval $\mathcal{I} \subset[0, \infty)$ we denote by $\Omega_{\mathcal{I}}^{j}(M, E)$ the image of the spectral projection of $\mathcal{B}^{2}$ corresponding to the eigenvalues whose absolute value lies in $\mathcal{I}$, cf Section 6.10 and Section 7.5 for details. The space $\Omega_{\mathcal{I}}^{j}(M, E)$ contains the span of the generalized eigenforms of $\mathcal{B}^{2}$ corresponding to eigenvalues whose absolute value lies in $\mathcal{I}$ and coincides with this span if the interval $\mathcal{I}$ is bounded. In particular, if $\mathcal{I}$ is bounded, then the dimension of $\Omega_{\mathcal{I}}^{j}(M, E)$ is finite. Note that, since $\mathcal{B}^{2}$ and $\nabla$ commute, the space $\Omega_{\mathcal{I}}^{j}(M, E)$ is a subcomplex of the de Rham complex $\Omega^{\bullet}(M, E)$.

For each $\lambda \geq 0$, we have

$$
\Omega^{\bullet}(M, E)=\Omega_{[0, \lambda]}^{\bullet}(M, E) \oplus \Omega_{(\lambda, \infty)}^{\bullet}(M, E) .
$$

The complex $\Omega_{(\lambda, \infty)}^{\bullet}(M, E)$ is clearly acyclic. Hence, the cohomology $H_{[0, \lambda]}^{\bullet}(M, E)$ of the complex $\Omega_{[0, \lambda]}^{\bullet}(M, E)$ is naturally isomorphic to the cohomology $H^{\bullet}(M, E)$ of $\Omega^{\bullet}(M, E)$. Further, as $\Gamma$ commutes with $\mathcal{B}^{2}$, it preserves the space $\Omega_{[0, \lambda]}(M, E)$ and the restriction $\Gamma_{[0, \lambda]}$ of $\Gamma$ to this space is a chirality operator on $\Omega_{[0, \lambda]}^{\bullet}(M, E)$.

To define the refined analytic torsion we need to introduce the notion of a graded determinant of $\mathcal{B}$. For every interval $\mathcal{I} \subset[0, \infty)$ and for each $k=0, \ldots, d$, set

$$
\begin{aligned}
& \Omega_{+, \mathcal{I}}^{k}(M, E):=\operatorname{Ker}(\nabla \Gamma) \cap \Omega_{\mathcal{I}}^{k}(M, E)=(\Gamma(\operatorname{Ker} \nabla)) \cap \Omega_{\mathcal{I}}^{k}(M, E) ; \\
& \Omega_{-, \mathcal{I}}^{k}(M, E):=\operatorname{Ker}(\Gamma \nabla) \cap \Omega_{\mathcal{I}}^{k}(M, E)=\operatorname{Ker} \nabla \cap \Omega_{\mathcal{I}}^{k}(M, E) .
\end{aligned}
$$

If $0 \notin \mathcal{I}$, then, clearly,

$$
\Omega_{\mathcal{I}}^{k}(M, E)=\Omega_{+, \mathcal{I}}^{k}(M, E) \oplus \Omega_{-, \mathcal{I}}^{k}(M, E) .
$$

The latter decomposition is considered as a grading on $\Omega_{\mathcal{I}}^{\bullet}(M, E)$. As both, $\Gamma$ and $\nabla$, commute with $\mathcal{B}^{2}$, we conclude that for all $j=0, \ldots, d$,

$$
\begin{gathered}
\Gamma: \Omega_{ \pm, \mathcal{I}}^{k}(M, E) \longrightarrow \Omega_{\mp, \mathcal{I}}^{d-k}(M, E), \\
\nabla: \Omega_{ \pm, \mathcal{I}}^{k}(M, E) \longrightarrow \Omega_{\mp, \mathcal{I}}^{k+1}(M, E) .
\end{gathered}
$$


Denote by $\mathcal{B}_{\text {even }}^{\mathcal{I}}$ and $\mathcal{B}_{\text {even }}^{ \pm, \mathcal{I}}$ the restrictions of $\mathcal{B}$ to $\Omega_{\mathcal{I}}^{\text {even }}(M, E):=\bigoplus_{p=0}^{r-1} \Omega_{\mathcal{I}}^{2 p}(M, E)$ and $\Omega_{\mathcal{I}}^{\text {even, } \pm}(M, E):=\bigoplus_{p=0}^{r-1} \Omega_{ \pm, \mathcal{I}}^{2 p}(M, E)$, respectively.

Let $\theta \in(-\pi, 0)$ be an Agmon angle for $\mathcal{B}^{\mathcal{I}}$, cf Definition 6.3. For each $\mathcal{I}$ with $0 \notin \mathcal{I}$ define the graded determinant of the operator $\mathcal{B}_{\text {even }}^{\mathcal{I}}$ by the formula

$$
\operatorname{Det}_{\mathrm{gr}, \theta}\left(\mathcal{B}_{\text {even }}^{\mathcal{I}}\right) \stackrel{\text { Def }}{=} \operatorname{Det}_{\theta}\left(\mathcal{B}_{\text {even }}^{+, \mathcal{I}}\right) / \operatorname{Det}_{\theta}\left(-\mathcal{B}_{\text {even }}^{-, \mathcal{I}}\right),
$$

where $\operatorname{Det}_{\theta}$ denotes the $\zeta$-regularized determinant, cf Section 6 and Section 7 for details. One verifies easily that for any $0 \leq \lambda \leq \mu<\infty$,

$$
\operatorname{Det}_{\mathrm{gr}, \theta}\left(\mathcal{B}_{\text {even }}^{(\lambda, \infty)}\right)=\operatorname{Det}_{\mathrm{gr}, \theta}\left(\mathcal{B}_{\text {even }}^{(\lambda, \mu]}\right) \cdot \operatorname{Det}_{\mathrm{gr}, \theta}\left(\mathcal{B}_{\text {even }}^{(\mu, \infty)}\right)
$$

For any given $\lambda \geq 0$, denote by $\rho_{\Gamma_{[0, \lambda]}}$ the refined torsion of the finite dimensional complex $\Omega_{[0, \lambda]}^{\bullet}(M, E)$ and the chirality operator $\Gamma_{[0, \lambda]}$. In view of (1-2) and (1-3), the product

$$
\rho=\rho\left(\nabla, g^{M}\right):=\operatorname{Det}_{\mathrm{gr}, \theta}\left(\mathcal{B}_{\text {even }}^{(\lambda, \infty)}\right) \cdot \rho_{\Gamma_{[0, \lambda]}} \in \operatorname{Det}\left(H^{\bullet}(M, E)\right)
$$

is independent of the choice of $\lambda \geq 0$. It is also independent of the choice of the Agmon angle $\theta \in(-\pi, 0)$ of $\mathcal{B}_{\text {even }}$.

\subsection{The metric anomaly of $\rho\left(\nabla, g^{M}\right)$}

The element $\rho\left(\nabla, g^{M}\right)$ is very close to our notion of the refined analytic torsion. However, in general, it is not a differential invariant of the flat bundle $E$, since it does depend on the choice of the Riemannian metric $g^{M}$. To compute the metric anomaly of $\rho\left(\nabla, g^{M}\right)$ we show (cf Proposition 8.1) that

$$
\operatorname{Det}_{\mathrm{gr}, \theta}\left(\mathcal{B}_{\mathrm{even}}^{(\lambda, \infty)}\right)=\exp \left(\xi_{\lambda}-i \pi \eta_{\lambda}-\frac{i \pi}{2} \sum_{j=0}^{d}(-1)^{j} j d_{j, \lambda}\right) .
$$

Here

$$
\xi_{\lambda}=\left.\frac{1}{2} \sum_{j=0}^{d-1}(-1)^{j+1} \frac{d}{d s}\right|_{s=0} \zeta_{2 \theta}\left(s,\left.(\Gamma \nabla)^{2}\right|_{\Omega_{+,(\lambda, \infty)}^{j}(M, E)}\right),
$$

where $\theta \in(-\pi / 2,0)$ is an Agmon angle for $\mathcal{B}$ such that there are no eigenvalues of $\mathcal{B}$ in the solid angles $L_{(-\pi / 2, \theta]}$ and $L_{(\pi / 2, \theta+\pi]}$,

$$
\eta_{\lambda}=\eta\left(\mathcal{B}_{\text {even }}^{(\lambda, \infty)}\right)
$$

is the $\eta$-invariant of $\mathcal{B}_{\text {even }}^{(\lambda, \infty)}$, and

$$
d_{j, \lambda}:=\operatorname{dim} \Omega_{[0, \lambda]}^{j}(M, E) .
$$


We then analyze the dependence on the Riemannian metric separately for $\xi_{\lambda} \cdot \rho_{\Gamma_{[0, \lambda]}}$ and $\eta_{\lambda}$. The metric anomaly for $\eta_{\lambda}$ has been already computed by Gilkey [19]. Using his result, we compute the metric anomaly of $\rho\left(\nabla, g^{M}\right)$. The refined analytic torsion is then defined by correcting $\rho\left(\nabla, g^{M}\right)$ by its anomaly. More precisely, we prove (cf Theorem 9.6) the following Theorem.

Theorem 1.6 Let $E$ be a flat vector bundle over a closed oriented odd-dimensional manifold $M$ and let $\nabla$ denote the flat connection on $E$. Let $N$ be an oriented manifold whose oriented boundary is the disjoint union of two copies of $M$. Then the product

$$
\rho\left(\nabla, g^{M}\right) \cdot e^{i \pi(\operatorname{rank} E) \eta_{\text {trivial }}} \in \operatorname{Det}\left(H^{\bullet}(M, E)\right),
$$

is independent of the metric $g^{M}$. Here

$$
\eta_{\text {trivial }}=\frac{1}{2} \eta\left(0, \mathcal{B}_{\text {even }}^{\text {trivial }}\right)
$$

is the half of the value at 0 of the $\eta$-function of the odd signature operator $\mathcal{B}_{\text {even }}^{\text {trivial }}$ associated to the trivial connection on the trivial complex line bundle $M \times \mathbb{C} \rightarrow M$.

In particular if $\operatorname{dim} M \equiv 1(\bmod 4)$ then $\eta_{\text {trivial }}=0, c f[1]$, and hence $\rho\left(\nabla, g^{M}\right)$ is independent of $g^{M}$.

\subsection{Definition of the refined analytic torsion}

We now define the refined analytic torsion $\rho_{\text {an }}=\rho_{\text {an }}(\nabla)$ to be the element (1-6) of $\operatorname{Det}\left(H^{\bullet}(M, E)\right)$. It is independent of the choice of the Agmon angle $\theta \in(-\pi, 0)$ and of the metric $g^{M}$.

In Remark 9.9 we also suggest an alternative definition of the refined analytic torsion, which is more convenient for some applications.

\subsection{The Ray-Singer metric of the refined analytic torsion}

Let $\|\cdot\|_{\operatorname{Det}(H \bullet(M, E))}^{\mathrm{RS}}$ denote the Ray-Singer norm on the determinant line given by $\operatorname{Det}\left(H^{\bullet}(M, E)\right)$, cf Bismut-Zhang [4] and Section 11.2. In Section 10, we compute the refined analytic torsion associated to the connection $\nabla^{\prime}$ on $E$ dual to a given connection $\nabla$. In Section 11, we use this calculation to calculate the Ray-Singer norm of the refined analytic torsion. More precisely we prove (cf Theorem 11.3) the following

Theorem 1.9 Let $E$ be a complex vector bundle over a closed, oriented, odddimensional manifold $M$ and let $\nabla$ be a flat connection on $E$. Then

$$
\left\|\rho_{\text {an }}\right\|_{\operatorname{Det}\left(H^{\bullet}(M, E)\right)}^{\mathrm{RS}}=e^{\pi \operatorname{Im} \eta\left(\mathcal{B}_{\text {even }}\left(\nabla, g^{M}\right)\right)} .
$$


In particular, if $\nabla$ is a Hermitian connection, then the operator $\mathcal{B}_{\text {even }}\left(\nabla, g^{M}\right)$ is selfadjoint, hence, its $\eta$-invariant $\eta\left(\mathcal{B}_{\text {even }}\left(\nabla, g^{M}\right)\right)$ is real, and

$$
\left\|\rho_{\mathrm{an}}\right\|_{\operatorname{Det}\left(H^{\bullet}(M, E)\right)}^{\mathrm{RS}}=1 \text {. }
$$

\subsection{Project "Refined analytic torsion"}

The present paper is the central part of an ongoing project whose aim is to refine the Ray-Singer torsion [29] and establish the relationship of the refined analytic torsion with the refined combinatorial torsion introduced by Turaev $[35 ; 36]$ and in a more general setting by Farber and Turaev $[17 ; 18]$. In this subsection, we briefly describe various results of ours as well as related results obtained by others and explain how they fit together.

Recall that the Ray-Singer torsion is defined using the square roots of the determinants of the Laplacians on forms. The main idea of the construction of the refined analytic torsion is to replace these square roots by the graded determinant of the restriction $\mathcal{B}_{\text {even }}$ of the odd signature operator $\mathcal{B}$ to even forms. Recall that when the connection $\nabla$ is Hermitian, then $\mathcal{B}^{2}=\Delta$. Thus, roughly speaking, we replace the square root of the determinant of the Laplacian by the determinant of the square root of the Laplacian.

In our previous paper [9] we treated in detail the case when $\mathcal{B}$ is bijective and $\nabla$ is acyclic. In this case, up to a correction by the multiplicative anomaly, the refined analytic torsion is equal to the graded determinant of $\mathcal{B}_{\text {even }}$. In the present paper we use the results of [9] as one of the main ingredients for the construction of the refined analytic torsion in the general case, when $\mathcal{B}$ is not necessarily bijective. To do this we fix a number $0 \leq \lambda<\infty$ and split the de Rham complex $\Omega^{\bullet}(M, E)$ into the direct sum

$$
\Omega^{\bullet}(M, E)=\Omega_{[0, \lambda]}^{\bullet}(M, E) \oplus \Omega_{(\lambda, \infty)}^{\bullet}(M, E)
$$

of spectral subspaces of the operator $\mathcal{B}^{2}$ corresponding to the small and the large eigenvalues of $\mathcal{B}^{2}$ respectively, cf Section 1.4. The space $\Omega_{[0, \lambda]}^{\bullet}(M, E)$ is a finite dimensional subcomplex, while the restriction $\mathcal{B}^{(\lambda, \infty)}$ of $\mathcal{B}$ to $\Omega_{(\lambda, \infty)}^{\bullet}(M, E)$ is bijective. The Hodge $*$-operator induces an involution on $\Omega_{[0, \lambda]}^{\bullet}(M, E)-$ the chirality operator $\Gamma_{[0, \lambda]}$. The main step in the present paper is a construction of the refined torsion of a finite dimensional complex endowed with a chirality operator - see Sections 2-5. We then define the refined analytic torsion $\rho_{\text {an }}$ (up to the metric anomaly) to be the product of the graded determinant of the operator $\mathcal{B}_{\text {even }}^{(\lambda, \infty)}$ and the refined torsion of the finite dimensional complex $\Omega_{[0, \lambda]}^{\bullet}(M, E)$ and show that it does not depend on the choice of $\lambda$. The study of the properties of $\rho_{\text {an }}$ in this and the subsequent papers relies heavily on the results about the graded determinant of $\mathcal{B}_{\text {even }}$ obtained in [9]. 
One of the main results of the present paper is the calculation of the Ray-Singer norm of the refined analytic torsion, cf Theorem 1.9. In particular, if the connection $\nabla$ is Hermitian, the norm of $\rho_{\text {an }}$ is equal to one. This result justifies calling $\rho_{\text {an }}$ the refined analytic torsion. Many other interesting and important properties of the refined analytic torsion were established since the first version [7] of this paper was released in 2005. We now briefly describe some of these results.

Denote by $\operatorname{Rep}\left(\pi_{1}(M), \mathbb{C}^{n}\right)$ the space of $n$-dimensional complex representations of the fundamental group $\pi_{1}(M)$ of $M$. For $\alpha \in \operatorname{Rep}\left(\pi_{1}(M), \mathbb{C}^{n}\right)$ we denote by $E_{\alpha}$ the flat vector bundle over $M$ whose monodromy is equal to $\alpha$. Let $\nabla_{\alpha}$ be the flat connection on $E_{\alpha}$. The refined analytic torsion $\rho_{\text {an }}(\alpha)=\rho_{\text {an }}\left(\nabla_{\alpha}\right)$ is an element of the determinant line Det $\left(H^{\bullet}\left(M, E_{\alpha}\right)\right)$. The disjoint union of the lines $\operatorname{Det}\left(H^{\bullet}\left(M, E_{\alpha}\right)\right)$, $\left(\alpha \in \operatorname{Rep}\left(\pi_{1}(M), \mathbb{C}^{n}\right)\right)$, forms a line bundle $\operatorname{Det} \rightarrow \operatorname{Rep}\left(\pi_{1}(M), \mathbb{C}^{n}\right)$, called the determinant line bundle, $\mathrm{cf}$ [3, Section 9.7]. It admits a nowhere vanishing section, given by the Farber-Turaev torsion, and, hence, has a natural structure of a trivializable holomorphic bundle.

In our subsequent paper [8] we prove that $\rho_{\mathrm{an}}(\alpha)$ is a nowhere vanishing holomorphic section of the bundle Det. It means that the ratio of the refined analytic and the Farber-Turaev torsions is a holomorphic function on $\operatorname{Rep}\left(\pi_{1}(M), \mathbb{C}^{n}\right)$. For an acyclic representation $\alpha$, the determinant line $\operatorname{Det}\left(H^{\bullet}\left(M, E_{\alpha}\right)\right)$ is canonically isomorphic to $\mathbb{C}$ and $\rho_{\text {an }}(\alpha)$ can be viewed as a nonzero complex number. Then $\rho_{\text {an }}(\alpha)$ becomes a holomorphic function on the open set $\operatorname{Rep}_{0}\left(\pi_{1}(M), \mathbb{C}^{n}\right) \subset \operatorname{Rep}\left(\pi_{1}(M), \mathbb{C}^{n}\right)$ of acyclic representations, whose absolute value is equal to the Ray-Singer torsion times $e^{\pi \operatorname{Im} \eta\left(\mathcal{B}_{\text {even }}\left(\nabla_{\alpha}, g^{M}\right)\right)}$ and whose phase is equal to $-\pi \operatorname{Re} \eta\left(\mathcal{B}_{\text {even }}\left(\nabla_{\alpha}, g^{M}\right)\right)+$ $\pi n \eta_{\text {trivial }}\left(g^{M}\right)$. In particular, when the representation $\alpha$ is unitary, the $\eta$-invariant is real and the absolute value of $\rho_{\mathrm{an}}(\alpha)$ is equal to the Ray-Singer torsion while the phase of $\rho_{\text {an }}(\alpha)$ is equal to $-\pi \eta\left(\mathcal{B}_{\text {even }}\left(\nabla_{\alpha}, g^{M}\right)\right)+\pi n \eta_{\text {trivial }}\left(g^{M}\right)$. The fact that the Ray-Singer torsion and the $\eta$-invariant can be combined into one holomorphic function allows one to use methods of complex analysis to study both invariants. In [8], using these methods, we compute the quotient of the refined analytic torsion and the Farber-Turaev torsion generalizing in this way the classical Cheeger-Müller theorem. As an application we establish a formula relating the $\eta$-invariant and the phase of the Farber-Turaev torsion extending earlier results of Farber [16]. The significance of this application stems from the fact that the $\eta$-invariant, defined in analytic terms, now can be studied using methods of combinatorial topology.

In our most recent paper [6] we compare the refined analytic torsion to the complex Ray-Singer torsion, recently introduced by Burghelea and Haller [12] which is a complex valued quadratic form on $\operatorname{Det}\left(H^{\bullet}(M, E)\right.$. In [6], we compute explicitly its value at the refined analytic torsion. As an application we obtain new results about the 
Burghelea-Haller torsion. In particular, we proved, up to a complex numbers of norm 1, the Burghelea-Haller conjecture concerning the value of their torsion for the FarberTuraev combinatorial torsion. In [23], Huang has proved the conjecture, up to sign, on connected components which contain a unitary representation. Recently, arguing as in Burghelea-Friedlander-Kappeler [11], Burghelea and Haller [13] have proved the above mentioned conjecture in full generality, again up to sign. Independently, a different proof of the same conjecture has been provided by Su and Zhang [34].

In [23; 22], Huang studies further properties of $\rho_{\text {an }}$ such as the product formula, and explicitly computes $\rho_{\text {an }}$ for lens spaces, answering several questions raised in [9].

\section{Acknowledgements}

The first author was supported in part by the NSF grant DMS-0204421. The second author was supported in part by the Swiss National Science foundation, the programme SPECT, and the European Community through the FP6 Marie Curie RTN ENIGMA (MRTN-CT-2004-5652). Both authors would like to thank Guangxiang Su for pointing out a sign mistake in a preliminary version of the paper and Michael Farber for valuable comments. The first author would like to thank the Institut des Hautes Études Scientifiques, where part of this work was completed, for hospitality and providing excellent working conditions.

\section{The determinant line of a finite dimensional complex}

In this section we review some standard material about determinant lines of finite dimensional spaces and complexes and define a sign refined version of the isomorphism between the determinant line of a complex and the determinant line of its cohomology similar to the one introduced by Turaev, [35] (see also [36], [37], and [18]). We also discuss some properties of this isomorphism.

Let $\mathbf{k}$ be a field of characteristic zero.

\subsection{Determinant lines}

Let $V$ be a $\mathbf{k}$-vector space of dimension $\operatorname{dim} V=n$. The determinant line of $V$ is the line $\operatorname{Det}(V):=\Lambda^{n} V$, where $\Lambda^{n} V$ denotes the $n$th exterior power of $V$. By definition, we set $\operatorname{Det}(0):=\mathbf{k}$. 
More generally, if $V^{\bullet}=V^{0} \oplus V^{1} \oplus \cdots \oplus V^{d}$ is a graded $\mathbf{k}$-vector space, we define the determinant line of $V^{\bullet}$ by the formula

$$
\operatorname{Det}\left(V^{\bullet}\right):=\bigotimes_{j=0}^{d} \operatorname{Det}\left(V^{j}\right)^{(-1)^{j}},
$$

where for a $\mathbf{k}$-line $L$ we denote by $L^{-1}:=\operatorname{Hom}_{\mathbf{k}}(L, \mathbf{k})$ the dual line.

If $L$ is a $\mathbf{k}$-line and $l \in L$ is a nonzero element, we denote by $l^{-1} \in L^{-1}$ the unique $\mathbf{k}$-linear map $L \rightarrow \mathbf{k}$ such that $l^{-1}(l)=1$.

\subsection{The determinant line of a finite dimensional complex}

Let

$$
\left(C^{\bullet}, \partial\right): \quad 0 \rightarrow C^{0} \stackrel{\partial}{\longrightarrow} C^{1} \stackrel{\partial}{\longrightarrow} \cdots \stackrel{\partial}{\longrightarrow} C^{d} \rightarrow 0
$$

be a complex of finite dimensional $\mathbf{k}$-vector spaces. We call the integer $d$ the length of the complex $\left(C^{\bullet}, \partial\right)$ and we denote by $H^{\bullet}(\partial)=\bigoplus_{i=0}^{d} H^{i}(\partial)$ the cohomology of $\left(C^{\bullet}, \partial\right)$. Set

$$
\begin{gathered}
\operatorname{Det}\left(C^{\bullet}\right):=\bigotimes_{j=0}^{d} \operatorname{Det}\left(C^{j}\right)^{(-1)^{j}}, \\
\operatorname{Det}\left(H^{\bullet}(\partial)\right):=\bigotimes_{j=0}^{d} \operatorname{Det}\left(H^{j}(\partial)\right)^{(-1)^{j}} .
\end{gathered}
$$

\subsection{The determinant line of a direct sum}

For two finite dimensional $\mathbf{k}$-vector spaces $V$ and $W$ we define the canonical fusion isomorphism

$$
\mu_{V, W}: \operatorname{Det}(V) \otimes \operatorname{Det}(W) \longrightarrow \operatorname{Det}(V \oplus W)
$$

by the formula

$$
\begin{aligned}
\mu_{V, W}:\left(v_{1} \wedge v_{2} \wedge \cdots \wedge v_{k}\right) \otimes\left(w_{1}\right. & \left.\wedge w_{2} \wedge \cdots \wedge w_{l}\right) \\
& \mapsto v_{1} \wedge v_{2} \wedge \cdots \wedge v_{k} \wedge w_{1} \wedge w_{2} \wedge \cdots \wedge w_{l},
\end{aligned}
$$

where $k=\operatorname{dim} V, l=\operatorname{dim} W, v_{j} \in V, w_{j} \in W$. Clearly, for $v \in \operatorname{Det}(V), w \in \operatorname{Det}(W)$ we have

$$
\mu_{V, W}(v \otimes w)=(-1)^{\operatorname{dim} V \cdot \operatorname{dim} W} \mu_{W, V}(w \otimes v) .
$$


By a slight abuse of notation, we denote by

$$
\mu_{V, W}^{-1}: \operatorname{Det}(V)^{-1} \otimes \operatorname{Det}(W)^{-1} \longrightarrow \operatorname{Det}(V \oplus W)^{-1}
$$

the transpose of the inverse of $\mu_{V, W}$. It than follows that, for any $v \in \operatorname{Det}(V)$ and $w \in \operatorname{Det}(W)$,

$$
\mu_{V, W}^{-1}\left(v^{-1} \otimes w^{-1}\right)=\left(\mu_{V, W}(v \otimes w)\right)^{-1} .
$$

Similarly, if $V_{1}, \ldots, V_{r}$ are finite dimensional $\mathbf{k}$-vector spaces, we define an isomorphism

$$
\mu_{V_{1}, \ldots, V_{r}}: \operatorname{Det}\left(V_{1}\right) \otimes \cdots \otimes \operatorname{Det}\left(V_{r}\right) \longrightarrow \operatorname{Det}\left(V_{1} \oplus \cdots \oplus V_{r}\right) .
$$

One easily checks that, for every $j \in 1, \ldots, r-1$,

$$
\begin{aligned}
& \mu_{V_{1}, \ldots, V_{r}}= \\
& \quad \mu_{V_{1}, \ldots, V_{j-1}, V_{j} \oplus V_{j+1}, V_{j+2}, \ldots, V_{r}} \circ\left(1 \otimes \cdots \otimes 1 \otimes \mu_{V_{j}, V_{j+1}} \otimes 1 \otimes \cdots \otimes 1\right) .
\end{aligned}
$$

\subsection{The isomorphism between the determinant line of a complex and the determinant line of its cohomology}

Fix a direct sum decomposition

$$
C^{j}=B^{j} \oplus H^{j} \oplus A^{j}, \quad j=0, \ldots, d,
$$

such that $B^{j} \oplus H^{j}=(\operatorname{Ker} \partial) \cap C^{j}$ and $B^{j}=\partial\left(C^{j-1}\right)=\partial\left(A^{j-1}\right)$, for all $j$. Note that $A^{d}=\{0\}$. Set $A^{-1}=\{0\}$. Then $H^{j}$ is naturally isomorphic to the cohomology $H^{j}(\partial)$ and $\partial$ defines an isomorphism $\partial: A^{j} \rightarrow B^{j+1}$.

For each $j=0, \ldots, d$, fix $c_{j} \in \operatorname{Det}\left(C^{j}\right)$ and $a_{j} \in \operatorname{Det}\left(A^{j}\right)$. Let $\partial\left(a_{j}\right) \in \operatorname{Det}\left(B^{j+1}\right)$ denote the image of $a_{j}$ under the map $\operatorname{Det}\left(A^{j}\right) \rightarrow \operatorname{Det}\left(B^{j+1}\right)$ induced by the isomorphism $\partial: A^{j} \rightarrow B^{j+1}$. Then, for each $j=0, \ldots, d$, there is a unique element $h_{j} \in \operatorname{Det}\left(H^{j}\right)$ such that

$$
c_{j}=\mu_{B^{j}, H^{j}, A^{j}}\left(\partial\left(a_{j-1}\right) \otimes h_{j} \otimes a_{j}\right) .
$$

Define the isomorphism

$$
\phi_{C^{\bullet}}=\phi_{\left(C^{\bullet}, \partial\right)}: \operatorname{Det}\left(C^{\bullet}\right) \longrightarrow \operatorname{Det}\left(H^{\bullet}(\partial)\right) \simeq \operatorname{Det}\left(H^{\bullet}\right),
$$

by the formula

$$
\phi_{C} \bullet: c_{0} \otimes c_{1}^{-1} \otimes \cdots \otimes c_{d}^{(-1)^{d}} \mapsto(-1)^{\mathcal{N}\left(C^{\bullet}\right)} h_{0} \otimes h_{1}^{-1} \otimes \cdots \otimes h_{d}^{(-1)^{d}},
$$


where

$$
\mathcal{N}\left(C^{\bullet}\right):=\frac{1}{2} \sum_{j=0}^{d} \operatorname{dim} A^{j} \cdot\left(\operatorname{dim} A^{j}+(-1)^{j+1}\right) .
$$

One easily checks that $\phi_{C} \bullet$ is independent of the choices of $c_{j}$ and $a_{j}$.

\section{Remark 2.5}

(a) We have

$$
\sum_{k=0}^{j}(-1)^{k} \operatorname{dim} C^{k}+(-1)^{j+1} \operatorname{dim} A^{j}=\sum_{k=0}^{j}(-1)^{k} \operatorname{dim} H^{k},
$$

since both sides of this equality are equal to the Euler characteristic of the complex

$$
0 \rightarrow C^{0} \stackrel{\partial}{\longrightarrow} C^{1} \stackrel{\partial}{\longrightarrow} \cdots \stackrel{\partial}{\longrightarrow} C^{j} \stackrel{\partial}{\longrightarrow} \partial\left(A^{j}\right) \rightarrow 0 .
$$

Hence, $\mathcal{N}\left(C^{\bullet}\right)$ can be expressed exclusively in terms of the dimensions of the spaces $C^{j}$ and $H^{j}(\partial)$.

(b) The isomorphism $\phi_{C}$ is a sign refined version of the standard construction, cf Milnor[25]. The idea to introduce a sign factor in the definition of $\phi_{C} \bullet$ is due to Turaev [35]. It allows to obtain various compatibility properties, cf, for example, Lemma 2.7 and Proposition 5.6 below. Our sign is slightly different from [35] but is consistent with Nicolaescu [26]. We refer the reader to Deligne [15] and Nicolaescu [26] for the motivation of this choice of sign, based on the theory of weighted determinant lines.

\subsection{The fusion isomorphism for graded vector spaces}

Let $V^{\bullet}=V^{0} \oplus V^{1} \oplus \cdots \oplus V^{d}$ and $W^{\bullet}=W^{0} \oplus W^{1} \oplus \cdots \oplus W^{d}$ be finite-dimensional graded $\mathbf{k}$-vector spaces. The fusion isomorphism

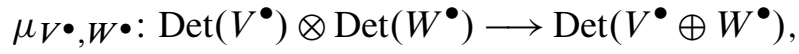

is defined by the formula

$$
\mu_{V^{\bullet}, W^{\bullet}}:=(-1)^{\mathcal{M}\left(V^{\bullet}, W^{\bullet}\right)} \bigotimes_{q=0}^{d} \mu_{V^{q}, W^{q}}^{(-1)^{q}}
$$


where $\mu_{V^{j}, W^{j}}^{+1}=\mu_{V^{j}, W^{j}}$ and $\mu_{V^{j}, W^{j}}^{-1}$ are defined in Section 2.3, and

$$
\mathcal{M}\left(V^{\bullet}, W^{\bullet}\right):=\sum_{0 \leq k<j \leq d} \operatorname{dim} V^{j} \cdot \operatorname{dim} W^{k} .
$$

The following lemma is a precise analogue of Farber-Turaev [18, Lemma 2.4].

Lemma 2.7 Let $\left(C^{\bullet}, \partial\right)$ and $\left(\widetilde{C}^{\bullet}, \widetilde{\partial}\right)$ be length $d$ complexes of finite dimensional $\mathbf{k}-$ vector spaces. Then the following diagram commutes:

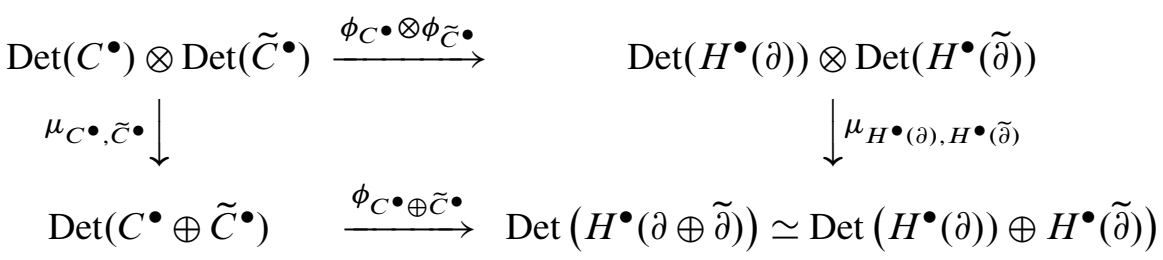

Proof As in (2-11), write

$$
C^{j}=B^{j} \oplus H^{j} \oplus A^{j}, \quad \widetilde{C}^{j}=\widetilde{B}^{j} \oplus \tilde{H}^{j} \oplus \tilde{A}^{j} .
$$

For each $j=0, \ldots, d$, choose

$$
\begin{array}{lll}
c_{j} \in \operatorname{Det}\left(C^{j}\right), & a_{j} \in \operatorname{Det}\left(A^{j}\right), & h_{j} \in \operatorname{Det}\left(H^{j}\right), \\
\tilde{c}_{j} \in \operatorname{Det}\left(\widetilde{C}^{j}\right), & \tilde{a}_{j} \in \operatorname{Det}\left(\tilde{A}^{j}\right), & \tilde{h}_{j} \in \operatorname{Det}\left(\tilde{H}^{j}\right),
\end{array}
$$

such that

$$
\begin{gathered}
c_{j}=\mu_{B^{j}, H^{j}, A^{j}}\left(\partial\left(a_{j-1}\right) \otimes h_{j} \otimes a_{j}\right), \\
\tilde{c}_{j}=\mu_{\widetilde{B}^{j}, \widetilde{H}^{j}, \tilde{A}^{j}}\left(\tilde{\partial}\left(\tilde{a}_{j-1}\right) \otimes \tilde{h}_{j} \otimes \tilde{a}_{j}\right) .
\end{gathered}
$$

Set

$$
\widehat{C}^{j}=C^{j} \oplus \widetilde{C}^{j}, \quad \hat{H}^{j}=H^{j} \oplus \tilde{H}^{j}, \quad \hat{A}^{j}=A^{j} \oplus \widetilde{A}^{j}, \quad \hat{B}^{j}=B^{j} \oplus \widetilde{B}^{j} .
$$

Also denote $\widehat{\partial}=\partial \oplus \widetilde{\partial}$. Further, set

$$
\widehat{c}_{j}=\mu_{C^{j}, \tilde{C}^{j}}\left(c_{j} \otimes \tilde{c}_{j}\right), \quad \widehat{a}_{j}=\mu_{A^{j}, \tilde{A}^{j}}\left(a_{j} \otimes \tilde{a}_{j}\right) .
$$

Then, for all $j=0, \ldots, d$, the unique element $\widehat{h}_{j} \in \operatorname{Det}\left(\hat{H}^{j}\right)$, satisfying

$$
\widehat{c}_{j}=\mu_{\widehat{B}^{j}, \hat{H}^{j}, \hat{A}^{j}}\left(\widehat{\partial}\left(\hat{a}_{j-1}\right) \otimes \widehat{h}_{j} \otimes \widehat{a}_{j}\right),
$$

is given by

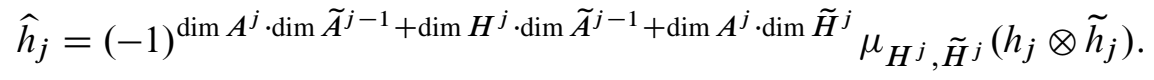


Set $c:=\bigotimes_{j=0}^{d} c_{j}^{(-1)^{j}}$ and define $\tilde{c}$ in a similar way. Then, by definitions (2-18) and $(2-23)$,

$$
\begin{aligned}
\mu_{C}, \tilde{C}^{\bullet}(c \otimes \tilde{c}) & =(-1)^{\mathcal{M}\left(C^{\bullet}, \tilde{C}^{\bullet}\right)} \bigotimes_{j=0}^{d} \mu_{C^{j}, \tilde{C}^{j}}\left(c_{j} \otimes \tilde{c}_{j}\right)^{(-1)^{j}} \\
& =(-1)^{\mathcal{M}\left(C^{\bullet}, \tilde{C}^{\bullet}\right)} \bigotimes_{j=0}^{d} \hat{c}_{j}^{(-1)^{j}} .
\end{aligned}
$$

From (2-23) and (2-25) we conclude that

$$
\phi_{C \bullet \oplus \tilde{C} \bullet} \circ \mu_{C}, \tilde{C} \bullet(c \otimes \tilde{c})=(-1)^{\mathcal{K}\left(C^{\bullet}, \tilde{C}^{\bullet}\right)} \bigotimes_{j=0}^{d} \mu_{H^{j}, \tilde{H}^{j}}\left(h_{j} \otimes \tilde{h}_{j}\right)^{(-1)^{j}}
$$

where

$$
\begin{aligned}
\mathcal{K}\left(C^{\bullet}, \widetilde{C}^{\bullet}\right)=\mathcal{N}\left(C^{\bullet} \oplus \widetilde{C}^{\bullet}\right)+\mathcal{M}\left(C^{\bullet}, \widetilde{C}^{\bullet}\right) \\
\quad+\sum_{j=0}^{d}\left[\operatorname{dim} A^{j} \cdot \operatorname{dim} \widetilde{A}^{j-1}+\operatorname{dim} H^{j} \cdot \operatorname{dim} \widetilde{A}^{j-1}+\operatorname{dim} A^{j} \cdot \operatorname{dim} \tilde{H}^{j}\right]
\end{aligned}
$$

Since, clearly,

$$
\begin{aligned}
\mu_{H^{\bullet}(\partial), H^{\bullet}(\tilde{\partial})} & \circ\left(\phi_{C} \bullet \otimes \phi_{\tilde{C}^{\bullet}}\right)(c \otimes \tilde{c}) \\
& =(-1)^{\mathcal{N}\left(C^{\bullet}\right)+\mathcal{N}\left(\tilde{C}^{\bullet}\right)+\mathcal{M}\left(H^{\bullet}, \tilde{H}^{\bullet}\right)} \bigotimes_{j=0}^{d} \mu_{H^{j}, \tilde{H}^{j}}\left(h_{j} \otimes \tilde{h}_{j}\right)^{(-1)^{j}},
\end{aligned}
$$

to prove the commutativity of the diagram (2-20) it remains to show that, mod 2 ,

$$
\begin{aligned}
\mathcal{N}\left(C^{\bullet} \oplus \widetilde{C}^{\bullet}\right)+\mathcal{N}\left(C^{\bullet}\right)+\mathcal{N}\left(\widetilde{C}^{\bullet}\right)+\mathcal{M}\left(H^{\bullet}, \tilde{H}^{\bullet}\right)+\mathcal{M}\left(C^{\bullet}, \widetilde{C}^{\bullet}\right) \\
\equiv \sum_{j=0}^{d}\left[\operatorname{dim} A^{j} \cdot \operatorname{dim} \widetilde{A}^{j-1}+\operatorname{dim} H^{j} \cdot \operatorname{dim} \tilde{A}^{j-1}+\operatorname{dim} A^{j} \cdot \operatorname{dim} \widetilde{H}^{j}\right]
\end{aligned}
$$

Using the identity

$$
\frac{(x+y)\left(x+y+(-1)^{j}\right)}{2}-\frac{x\left(x+(-1)^{j}\right)}{2}-\frac{y\left(y+(-1)^{j}\right)}{2}=x y,
$$


where $x, y \in \mathbf{k}, j \in \mathbb{Z}_{\geq 0}$, we obtain

$$
\mathcal{N}\left(C^{\bullet} \oplus \tilde{C}^{\bullet}\right)-\mathcal{N}\left(C^{\bullet}\right)-\mathcal{N}\left(\widetilde{C}^{\bullet}\right)=\sum_{j=0}^{d} \operatorname{dim} A^{j} \cdot \operatorname{dim} \tilde{A}^{j}
$$

On the other hand, using (2-21) and the equalities $\operatorname{dim} \widetilde{A}^{j-1}=\operatorname{dim} \widetilde{B}^{j}$ we see that the following equality holds modulo 2

$$
\begin{aligned}
& \sum_{j=0}^{d}\left[\operatorname{dim} A^{j} \cdot \operatorname{dim} \widetilde{A}^{j-1}+\operatorname{dim} A^{j} \cdot \operatorname{dim} \tilde{H}^{j}\right] \\
& \quad=\sum_{j=0}^{d} \operatorname{dim} A^{j} \cdot\left(\operatorname{dim} \tilde{A}^{j-1}+\operatorname{dim} \tilde{H}^{j}\right) \\
& \quad \equiv \sum_{j=0}^{d} \operatorname{dim} A^{j} \cdot\left(\operatorname{dim} \tilde{A}^{j}+\operatorname{dim} \widetilde{C}^{j}\right) \\
& \quad=\sum_{j=0}^{d} \operatorname{dim} A^{j} \cdot \operatorname{dim} \tilde{A}^{j}+\sum_{j=0}^{d} \operatorname{dim} A^{j} \cdot \operatorname{dim} \widetilde{C}^{j} .
\end{aligned}
$$

By (2-16),

$$
\operatorname{dim} A^{j} \equiv \sum_{k=0}^{j}\left(\operatorname{dim} H^{k}+\operatorname{dim} C^{k}\right)
$$

A similar equality holds for $\operatorname{dim} \widetilde{A}^{j}$. Hence, we get from (2-32)

$$
\begin{aligned}
& \sum_{j=0}^{d}\left[\operatorname{dim} A^{j} \cdot \operatorname{dim} \tilde{A}^{j-1}+\operatorname{dim} A^{j} \cdot \operatorname{dim} \tilde{H}^{j}\right] \equiv \\
& \sum_{j=0}^{d} \operatorname{dim} A^{j} \cdot \operatorname{dim} \tilde{A}^{j}+\sum_{0 \leq k \leq j \leq d} \operatorname{dim} C^{k} \cdot \operatorname{dim} \widetilde{C}^{j}+\sum_{0 \leq k \leq j \leq d} \operatorname{dim} H^{k} \cdot \operatorname{dim} \widetilde{C}^{j} .
\end{aligned}
$$

Similarly,

(2-34) $\sum_{j=0}^{d} \operatorname{dim} H^{j} \cdot \operatorname{dim} \widetilde{A}^{j-1}=\sum_{0 \leq k<j \leq d} \operatorname{dim} H^{j} \cdot \operatorname{dim} \widetilde{C}^{k}+\sum_{0 \leq k<j \leq d} \operatorname{dim} H^{j} \cdot \operatorname{dim} \widetilde{H}^{k}$ 
Combining (2-19), (2-33), and (2-34) we obtain that modulo 2

$$
\begin{aligned}
& \sum_{j=0}^{d}\left[\operatorname{dim} A^{j} \cdot \operatorname{dim} \widetilde{A}^{j-1}+\operatorname{dim} H^{j} \cdot \operatorname{dim} \widetilde{A}^{j-1}+\operatorname{dim} A^{j} \cdot \operatorname{dim} \tilde{H}^{j}\right] \\
& \quad+\mathcal{M}\left(C^{\bullet}, \widetilde{C}^{\bullet}\right)+\mathcal{M}\left(H^{\bullet}, \tilde{H}^{\bullet}\right) \\
& \equiv \sum_{j=0}^{d} \operatorname{dim} A^{j} \cdot \operatorname{dim} \tilde{A}^{j}+\sum_{j, k=0}^{d} \operatorname{dim} C^{k} \operatorname{dim} \tilde{C}^{j}+\sum_{j, k=0}^{d} \operatorname{dim} H^{k} \operatorname{dim} \widetilde{C}^{j} \\
& \equiv \sum_{j=0}^{d} \operatorname{dim} A^{j} \cdot \operatorname{dim} \tilde{A}^{j}+\left(\sum_{k=0}^{d} \operatorname{dim} C^{k}\right) \cdot\left(\sum_{j=0}^{d} \operatorname{dim} \widetilde{C}^{j}\right) \\
& \quad+\left(\sum_{k=0}^{d} \operatorname{dim} H^{k}\right) \cdot\left(\sum_{j=0}^{d} \operatorname{dim} \tilde{C}^{j}\right) .
\end{aligned}
$$

Both $\sum_{k=0}^{d} \operatorname{dim} C^{k}$ and $\sum_{k=0}^{d} \operatorname{dim} H^{k}$ are equivalent modulo 2 to the Euler characteristic of the complex $\left(C^{\bullet}, \partial\right)$. Hence, we conclude that the left hand side of (2-35) is equivalent modulo 2 to

$$
\sum_{j=0}^{d} \operatorname{dim} A^{j} \cdot \operatorname{dim} \tilde{A}^{j} .
$$

Combining this with (2-31), we obtain (2-29).

\section{The determinant line of the dual complex}

In this section we introduce the dual of a complex and, for the case when the length of the complex is odd, construct a natural isomorphism between the determinant lines of a complex and that of its dual. We also show that this isomorphism is compatible with the canonical isomorphism (2-13).

Throughout the section, $\mathbf{k}$ is a field of characteristic zero endowed with an involutive automorphism

$$
\tau: \mathbf{k} \rightarrow \mathbf{k}
$$

The main examples are $\mathbf{k}=\mathbb{C}$ with $\tau$ being the complex conjugation and $\mathbf{k}=\mathbb{R}$ with $\tau$ being the identity map. 


\subsection{The determinant line of the $\tau$-dual space}

If $V, W$ are $\mathbf{k}$-vector spaces, a map $f: V \rightarrow W$ is said to be $\tau$-linear if

$$
f\left(x_{1} v_{1}+x_{2} v_{2}\right)=\tau\left(x_{1}\right) v_{1}+\tau\left(x_{2}\right) v_{2} \text { for any } v_{1}, v_{2} \in V, x_{1}, x_{2} \in \mathbf{k} .
$$

Let $V$ be an $n$-dimensional $\mathbf{k}$-vector space. The linear space $V^{*}=V^{*}$ of all $\tau$-linear maps $V \rightarrow \mathbf{k}$ is called the $\tau$-dual space to $V$. There is a natural $\tau$-linear isomorphism

$$
\alpha_{V}: \operatorname{Det}\left(V^{*}\right) \longrightarrow \operatorname{Det}(V)^{-1}
$$

defined by the formula

$$
\begin{aligned}
\left(\alpha_{V}\left(v^{1} \wedge \cdots \wedge v^{n}\right)\right) & \left(v_{1} \wedge \cdots \wedge v_{n}\right) \\
= & \sum_{\sigma}(-1)^{|\sigma|} \tau\left(v^{1}\left(v_{\sigma(1)}\right)\right) \cdot \tau\left(v^{2}\left(v_{\sigma(2)}\right)\right) \cdots \tau\left(v^{n}\left(v_{\sigma(n)}\right)\right)
\end{aligned}
$$

where $v_{1}, \ldots, v_{n} \in V, v^{1}, \ldots, v^{n} \in V^{*}$, and the sum is taken over all permutations $\sigma$ of $\{1, \ldots, n\}$. Similarly, we define the $\tau$-linear map

$$
\beta_{V}: \operatorname{Det}(V) \longrightarrow \operatorname{Det}\left(V^{*}\right)^{-1},
$$

defined by the formula

$$
\begin{aligned}
&\left(\beta_{V}\left(v_{1} \wedge \cdots \wedge v_{n}\right)\right)\left(v^{1} \wedge \cdots \wedge v^{n}\right) \\
&=(-1)^{n} \cdot \sum_{\sigma}(-1)^{|\sigma|} \tau\left(v^{1}\left(v_{\sigma(1)}\right)\right) \cdot \tau\left(v^{2}\left(v_{\sigma(2)}\right)\right) \cdots \tau\left(v^{n}\left(v_{\sigma(n)}\right)\right)
\end{aligned}
$$

Remark 3.2 The sign factor $(-1)^{n}$ in (3-4) simplifies the statements of various compatibility relations with the fusion isomorphism (2-4), cf below. It is motivated by the fact that, in (3-4), we interchange $v_{1} \wedge \cdots \wedge v_{n}$ and $v^{1} \wedge \cdots \wedge v^{n}$, which both are forms of degree $n$.

Formulae (3-2) and (3-4) can be simplified by choosing an appropriate basis. Let $e_{1}, \ldots, e_{n}$ be a basis of $V$. Denote by $e^{1}, \ldots, e^{n}$ the dual basis of $V^{*}$, ie, the unique set of elements of $V^{*}$ such that $e^{j}\left(e_{i}\right)=\delta_{i}^{j}$ for all $i, j=1, \ldots, n$. Then

(3-5) $\left(\alpha_{V}\left(e^{1} \wedge \ldots \wedge e^{n}\right)\right)\left(e_{1} \wedge \ldots \wedge e_{n}\right)=\tau\left(e^{1}\left(e_{1}\right)\right) \cdot \tau\left(e^{2}\left(e_{2}\right)\right) \ldots \tau\left(e^{n}\left(e_{n}\right)\right)$;

(3-6) $\left(\beta_{V}\left(e_{1} \wedge \cdots \wedge e_{n}\right)\right)\left(e^{1} \wedge \ldots \wedge e^{n}\right)=(-1)^{n} \cdot \tau\left(e^{1}\left(e_{1}\right)\right) \cdot \tau\left(e^{2}\left(e_{2}\right)\right) \ldots \tau\left(e^{n}\left(e_{n}\right)\right)$. 
Recall from Section 2.1 that for a nonzero element $v \in \operatorname{Det}(V)$ we denote by $v^{-1}$ the unique element of $(\operatorname{Det}(V))^{-1}$ such that $v^{-1}(v)=1$. It follows from (3-5) that

$$
\alpha_{V}^{-1}\left(\left(e_{1} \wedge \cdots \wedge e_{n}\right)^{-1}\right)=e^{1} \wedge \cdots \wedge e^{n} .
$$

Using (3-6) and (3-7) we conclude that for any $v \in \operatorname{Det}(V)$

$$
\left(\alpha_{V}^{-1}\left(v^{-1}\right)\right)^{-1}=(-1)^{\operatorname{dim} V} \beta_{V}(v) .
$$

Let $V$ and $W$ be k-vector spaces. From (2-5), (3-5), and (3-6), we obtain

$$
\left(\mu_{V, W}(v \otimes w)\right)^{-1}=\alpha_{V \oplus W} \circ \mu_{V^{*}, W^{*}}\left(\alpha_{V}^{-1}\left(v^{-1}\right) \otimes \alpha_{W}^{-1}\left(w^{-1}\right)\right),
$$

for any $v \in \operatorname{Det}(V), w \in \operatorname{Det}(W)$.

\subsection{The $\tau$-adjoint map}

Let $T: V \rightarrow W$ be a $\mathbf{k}$-linear map. The $\tau$-adjoint of $T$ is the linear map

$$
T^{*}: W^{*} \longrightarrow V^{*}
$$

such that

$$
\left(T^{*} w^{*}\right)(v)=w^{*}(T v), \text { for all } v \in V, w^{*} \in W^{*} .
$$

If $\operatorname{dim} V=\operatorname{dim} W$ then $T$ and $T^{*}$ induce $\mathbf{k}$-linear maps $\operatorname{Det}(V) \rightarrow \operatorname{Det}(W)$ and $\operatorname{Det}\left(W^{*}\right) \rightarrow \operatorname{Det}\left(V^{*}\right)$, which, by a slight abuse of notation, we also denote by $T$ and $T^{*}$ respectively. If $T$ is bijective then, for any nonzero $v \in \operatorname{Det}(V)$, we have

$$
T^{*} \alpha_{W}^{-1}\left((T v)^{-1}\right)=\alpha_{V}^{-1}\left(v^{-1}\right) .
$$

\subsection{The $\tau$-dual graded space}

Let now $V^{\bullet}=V^{0} \oplus V^{1} \oplus \cdots \oplus V^{d}$ be a finite dimensional graded $\mathbf{k}$-vector space. We define the $(\tau-)$ dual graded space $\widehat{V}=\widehat{V}^{0} \oplus \widehat{V}^{1} \oplus \cdots \oplus \widehat{V}^{d}$ by

$$
\widehat{V}^{j}:=\left(V^{d-j}\right)^{*}, \quad j=0, \ldots, d .
$$

Assume now that the number $d=2 r-1$ is odd. Then (3-1) and (3-3) induce a $\tau$-linear isomorphism

$$
\alpha_{V^{\bullet}}: \operatorname{Det}\left(V^{\bullet}\right) \longrightarrow \operatorname{Det}\left(\hat{V}^{\bullet}\right)
$$


defined by the formula

$$
\begin{aligned}
\alpha_{V} \cdot\left(v_{0} \otimes\left(v_{1}\right)^{-1}\right. & \left.\otimes \cdots \otimes\left(v_{d}\right)^{-1}\right) \\
& =(-1)^{\mathcal{M}\left(V^{\bullet}\right)} \cdot \alpha_{V^{d}}^{-1}\left(v_{d}^{-1}\right) \otimes \beta_{V^{d-1}}\left(v_{d-1}\right) \otimes \cdots \otimes \beta_{V^{0}}\left(v_{0}\right),
\end{aligned}
$$

where $v_{j} \in \operatorname{Det}\left(V^{j}\right)(j=0, \ldots, d)$ and

$$
\mathcal{M}\left(V^{\bullet}\right)=\mathcal{M}\left(V^{\bullet}, V^{\bullet}\right)=\sum_{0 \leq j<k \leq d} \operatorname{dim} V^{j} \cdot \operatorname{dim} V^{k},
$$

cf (2-19). We refer to Nicolaescu [26] for the motivation of the choice of the sign in (3-13).

\subsection{The dual complex}

Consider the complex (2-2) of finite dimensional $\mathbf{k}-$ vector spaces. The dual complex is the complex

$$
\left(\widehat{C}^{\bullet}, \partial\right): \quad 0 \rightarrow \widehat{C}^{0} \stackrel{\partial^{*}}{\longrightarrow} \widehat{C}^{1} \stackrel{\partial^{*}}{\longrightarrow} \cdots \stackrel{\partial^{*}}{\longrightarrow} \widehat{C}^{d} \rightarrow 0,
$$

where $\widehat{C}^{j}=\left(C^{d-j}\right)^{*}$ and $\partial^{*}$ is the $\tau$-adjoint of $\partial$. Then the cohomology $H^{j}\left(\partial^{*}\right)$ of $\widehat{C}^{\bullet}$ is naturally isomorphic to the $\tau$-dual space to $H^{d-j}(\partial)(j=0, \ldots, d)$. Hence, if the length $d$ of the complex $C^{\bullet}$ is odd, then, by (3-12), we obtain $\tau$-linear isomorphisms

$$
\begin{aligned}
\alpha_{C} \bullet: \operatorname{Det}\left(C^{\bullet}\right) & \longrightarrow \operatorname{Det}\left(\hat{C}^{\bullet}\right), \\
\alpha_{H^{\bullet}(\partial)}: \operatorname{Det}\left(H^{\bullet}(\partial)\right) & \longrightarrow \operatorname{Det}\left(H^{\bullet}\left(\partial^{*}\right)\right) .
\end{aligned}
$$

Lemma 3.6 Let $\left(C^{\bullet}, \partial\right)$ be a complex of finite dimensional $\mathbf{k}$-vector spaces and assume that its length $d=2 r-1$ is odd. Then the following diagram commutes

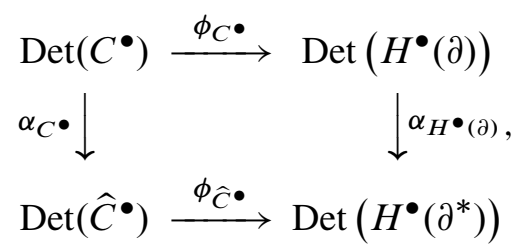

where the isomorphisms $\phi_{C} \bullet$ and $\phi_{\hat{C}}$ are as in (2-13).

Proof We shall use the notation of Section 2.4. For $j=0, \ldots, d$, set

$$
\widehat{A}^{j}:=\left(B^{d-j}\right)^{*}, \quad \widehat{B}^{j}:=\left(A^{d-j}\right)^{*}, \quad \hat{H}^{j}:=\left(H^{d-j}\right)^{*}
$$

and identify these spaces with subspaces of $\widehat{C}^{j}$ in a natural way. Then $\partial^{*}\left(\widehat{A}^{j}\right)=\widehat{B}^{j+1}$. 
Let $c_{j}, a_{j}, h_{j}(j=0, \ldots, d)$ be as in $(2-12)$. For each $j=0, \ldots, d$, set

$$
\begin{aligned}
& \widehat{c}_{j}=\alpha_{C^{d-j}}^{-1}\left(c_{d-j}^{-1}\right), \\
& \widehat{a}_{j}=\alpha_{B^{d-j}}^{-1}\left(\left(\partial a_{d-j-1}\right)^{-1}\right), \\
& \hat{h}_{j}=\alpha_{H^{d-j}}^{-1}\left(h_{d-j}^{-1}\right) .
\end{aligned}
$$

Then, from the equality (3-8), we obtain,

$$
\begin{aligned}
\beta_{C^{d-j}}\left(c_{d-j}\right) & =(-1)^{\operatorname{dim} C^{j}} \cdot \hat{c}_{j}^{-1}, \\
\beta_{H^{d-j}(\partial)}\left(h_{d-j}\right) & =(-1)^{\operatorname{dim} H^{j}(\partial)} \cdot \hat{h}_{j}^{-1} .
\end{aligned}
$$

Hence, from the definition (3-13), we get

$$
\begin{aligned}
\alpha_{H^{\bullet}(\partial)}\left(h_{0} \otimes h_{1}^{-1}\right. & \left.\otimes \cdots \otimes h_{d}^{-1}\right) \\
& =(-1)^{\mathcal{M}\left(H^{\bullet}(\partial)\right)+\sum_{p=0}^{r-1} \operatorname{dim} H^{2 p}(\partial)} \cdot \widehat{h}_{0} \otimes \widehat{h}_{1}^{-1} \otimes \cdots \otimes \widehat{h}_{d}^{-1}
\end{aligned}
$$

From the identity (3-11) and the definition (3-20) of $\hat{a}_{j}$, we get

$$
\partial^{*}\left(\widehat{a}_{j-1}\right)=\alpha_{A^{d-j}}^{-1}\left(a_{d-j}^{-1}\right), \quad j=1, \ldots, d .
$$

Hence, from (2-12) and (3-9), we obtain

$$
\begin{aligned}
\widehat{c}_{j} & =\alpha_{C^{d-j}}^{-1}\left(\mu_{B^{d-j}, H^{d-j}, A^{d-j}}\left(\partial\left(a_{d-j-1}\right) \otimes h_{d-j} \otimes a_{d-j}\right)\right)^{-1} \\
& =\mu_{\widehat{A}^{j}, \hat{H}^{j}, \widehat{B}^{j}}\left(\widehat{a}_{j} \otimes \hat{h}_{j} \otimes \partial^{*}\left(\widehat{a}_{j-1}\right)\right) .
\end{aligned}
$$

Using (2-6), we now conclude that

$$
\widehat{c}_{j}=(-1)^{\mathcal{G}_{j}} \cdot \mu_{\widehat{B}^{j}, \hat{H}^{j}, \hat{A}^{j}}\left(\partial^{*}\left(\widehat{a}_{j-1}\right) \otimes \hat{h}_{j} \otimes \widehat{a}_{j-1}\right),
$$

where

$$
\mathcal{G}_{j}=\operatorname{dim} \widehat{A}^{j} \cdot \operatorname{dim} \widehat{H}^{j}+\operatorname{dim} \widehat{A}^{j} \cdot \operatorname{dim} \widehat{A}^{j-1}+\operatorname{dim} \widehat{A}^{j-1} \cdot \operatorname{dim} \hat{H}^{j} .
$$

Thus, from (2-14), we obtain

$$
\phi_{\widehat{C}^{\bullet}}\left(\widehat{c}_{0} \otimes \widehat{c}_{1}^{-1} \otimes \cdots \otimes \hat{c}_{d}^{-1}\right)=(-1)^{\mathcal{N}\left(\hat{C}^{\bullet}\right)+\sum_{j=0}^{d} \mathcal{G}_{j}} \cdot \hat{h}_{0} \otimes \hat{h}_{1}^{-1} \otimes \cdots \otimes \hat{h}_{d}^{-1} .
$$

Hence, by (3-13) and (3-22),

$$
\begin{aligned}
\phi_{\hat{C}^{\bullet}} \circ \alpha_{C} \bullet & \left(c_{0} \otimes c_{1}^{-1} \otimes \cdots \otimes c_{d}^{-1}\right) \\
& =(-1)^{\mathcal{M}\left(C^{\bullet}\right)+\mathcal{N}\left(\hat{C}^{\bullet}\right)+\sum_{j=0}^{d} \mathcal{G}_{j}+\sum_{p=0}^{r-1} \operatorname{dim} C^{2 p}} \cdot \hat{h}_{0} \otimes \hat{h}_{1}^{-1} \otimes \cdots \otimes \widehat{h}_{d}^{-1} .
\end{aligned}
$$


From (3-13) and (3-23), we get

$$
\begin{aligned}
\alpha_{H \bullet(\partial)} \circ \phi_{C} & \left(c_{0} \otimes c_{1}^{-1} \otimes \cdots \otimes c_{d}^{-1}\right) \\
= & (-1)^{\mathcal{M}\left(H^{\bullet}(\partial)\right)+\mathcal{N}\left(C^{\bullet}\right)+\sum_{p=0}^{r-1} \operatorname{dim} H^{2 p}(\partial)} \cdot \hat{h}_{0} \otimes \hat{h}_{1}^{-1} \otimes \cdots \otimes \hat{h}_{d}^{-1}
\end{aligned}
$$

From (3-27) and (3-28), we conclude that to prove (3-17) it remains to show that, modulo 2 ,

$$
\begin{aligned}
\mathcal{M}\left(C^{\bullet}\right)+\mathcal{N}\left(\hat{C}^{\bullet}\right)+\sum_{j=0}^{d} \mathcal{G}_{j}+ & \sum_{p=0}^{r-1} \operatorname{dim} C^{2 p} \\
& \equiv \mathcal{M}\left(H^{\bullet}(\partial)\right)+\mathcal{N}\left(C^{\bullet}\right)+\sum_{p=0}^{r-1} \operatorname{dim} H^{2 p}(\partial) .
\end{aligned}
$$

Using the equality

$$
\operatorname{dim} \widehat{A}^{j}=\operatorname{dim} B^{d-j}=\operatorname{dim} A^{d-j-1},
$$

we easily see that $\mathcal{N}\left(\widehat{C}^{\bullet}\right)=\mathcal{N}\left(C^{\bullet}\right)$. In addition, note that if we set $\widetilde{C}^{\bullet}=C^{\bullet}$ in (2-29), then the right hand side of (2-29) is equal to $\sum_{j=0}^{d} \mathcal{G}_{j}$. Hence, (2-29) and (3-14) imply that (3-29) is equivalent to

$$
\mathcal{N}\left(C^{\bullet} \oplus C^{\bullet}\right)+\sum_{p=0}^{r-1}\left(\operatorname{dim} C^{2 p}-\operatorname{dim} H^{2 p}(\partial)\right) \equiv 0 \bmod 2
$$

By $(2-15)$,

$$
\mathcal{N}\left(C^{\bullet} \oplus C^{\bullet}\right) \equiv \sum_{j=0}^{d} \operatorname{dim} A^{j} \quad \bmod 2
$$

From (2-11) and the equality $\operatorname{dim} B^{j}=\operatorname{dim} A^{j-1}$ we conclude that

$$
\operatorname{dim} C^{2 p}-\operatorname{dim} H^{2 p}(\partial)=\operatorname{dim} A^{2 p}+\operatorname{dim} A^{2 p-1},
$$

and, hence,

$$
\sum_{p=0}^{r-1}\left(\operatorname{dim} C^{2 p}-\operatorname{dim} H^{2 p}(\partial)\right)=\sum_{j=0}^{d} \operatorname{dim} A^{j} .
$$

Combining (3-31) and (3-32) we obtain (3-30). 


\section{The refined torsion of a finite dimensional complex with a chirality operator}

On the de Rham complex of a Riemannian manifold acts a canonical involution $\Gamma$, called the chirality operator, cf [3, Chapter 3]. In this section we consider a finite dimensional complex with such an operator. We show that the chirality operator defines a canonical element, the refined torsion, of the determinant line of the cohomology of this complex and we study some properties of this element.

In this section $\mathbf{k}$ is a field of characteristic zero.

\subsection{The refined torsion associated to a chirality operator}

Let $d=2 r-1$ be an odd integer and let $\left(C^{\bullet}, \partial\right)$ be a length $d$ complex of finite dimensional $\mathbf{k}$-vector spaces. A chirality operator is an involution $\Gamma: C^{\bullet} \rightarrow C^{\bullet}$ such that $\Gamma\left(C^{j}\right)=C^{d-j}, j=0, \ldots, d$. For $c_{j} \in \operatorname{Det}\left(C^{j}\right)(j=0, \ldots, d)$ we denote by $\Gamma c_{j} \in \operatorname{Det}\left(C^{d-j}\right)$ the image of $c_{j}$ under the isomorphism $\operatorname{Det}\left(C^{j}\right) \rightarrow \operatorname{Det}\left(C^{d-j}\right)$ induced by $\Gamma$.

Fix nonzero elements $c_{j} \in \operatorname{Det}\left(C^{j}\right), j=0, \ldots, r-1$, and consider the element

$$
\begin{aligned}
&(4-1) \quad c_{\Gamma}:=(-1)^{\mathcal{R}\left(C^{\bullet}\right)} \cdot c_{0} \otimes c_{1}^{-1} \otimes \cdots \otimes c_{r-1}^{(-1)^{r-1}} \\
& \otimes\left(\Gamma c_{r-1}\right)^{(-1)^{r}} \otimes\left(\Gamma c_{r-2}\right)^{(-1)^{r-1}} \otimes \cdots \otimes\left(\Gamma c_{0}\right)^{-1,}
\end{aligned}
$$

of $\operatorname{Det}\left(C^{\bullet}\right)$, where

$$
\mathcal{R}\left(C^{\bullet}\right)=\frac{1}{2} \sum_{j=0}^{r-1} \operatorname{dim} C^{j} \cdot\left(\operatorname{dim} C^{j}+(-1)^{r+j}\right) .
$$

It follows from the definition of $c_{j}^{-1}$ that $c_{\Gamma}$ is independent of the choice of $c_{j}$ $(j=0, \ldots, r-1)$.

Remark 4.2 Using the isomorphisms $\Gamma: C^{j} \rightarrow C^{d-j}$ one can define a natural trace functional $\operatorname{Tr}$ : $\operatorname{Det}\left(C^{\bullet}\right) \rightarrow \mathbf{k}, \operatorname{cf}[26]$. The sign factor $(-1)^{\mathcal{R}\left(C^{\bullet}\right)}$ is defined so that the equality $\operatorname{Tr}\left(c_{\Gamma}\right)=1$ holds.

Definition 4.3 The refined torsion of the pair $\left(C^{\bullet}, \Gamma\right)$ is the element

$$
\rho_{\Gamma}=\rho_{C}, \Gamma=\phi_{C} \cdot\left(c_{\Gamma}\right),
$$

where $\phi_{C} \bullet$ is the canonical map defined in Section 2.4. 


\subsection{The norm of the refined torsion}

In this subsection we assume that $\mathbf{k}=\mathbb{R}$ or $\mathbb{C}$. Suppose that the spaces $C^{j}, j=0, \ldots, d$, are endowed with a Euclidean (if $\mathbf{k}=\mathbb{R}$ ) or a Hermitian (if $\mathbf{k}=\mathbb{C}$ ) scalar products $\langle\cdot, \cdot\rangle_{j}$. These scalar products induce a metric $\|\cdot\|_{\operatorname{Det}\left(C^{\bullet}\right)}$ on the determinant line $\operatorname{Det}\left(C^{\bullet}\right)$. Let $\|\cdot\|_{\operatorname{Det}\left(H^{\bullet}(\partial)\right)}$ be the metric on the determinant line $\operatorname{Det}\left(H^{\bullet}(\partial)\right)$ such that the canonical isomorphism $\phi_{C} \bullet$, defined in (2-13), is an isometry.

Lemma 4.5 Let $\langle\cdot, \cdot\rangle_{j}$ be scalar products on $C^{j}, j=0, \ldots, d$, such that the chirality operator $\Gamma$ is self-adjoint. Then

$$
\left\|\rho_{\Gamma}\right\|_{\operatorname{Det}(H \bullet(\partial))}=1
$$

Proof By definition,

$$
\left\|\rho_{\Gamma}\right\|_{\operatorname{Det}\left(H^{\bullet}(\partial)\right)}=\left\|c_{\Gamma}\right\|_{\operatorname{Det}\left(C^{\bullet}\right)} .
$$

Let $\|\cdot\|_{j}$ denote the norm on $\operatorname{Det}\left(C^{j}\right)$ induced by $\langle\cdot, \cdot\rangle_{j}$. Since $\Gamma$ is a self-adjoint involution it is also a unitary operator, ie, for every $x \in \operatorname{Det}\left(C^{j}\right)$ we have $\|\Gamma x\|_{d-j}=$ $\|x\|_{j}$. Hence, from (4-1) we get $\left\|c_{\Gamma}\right\|_{\operatorname{Det}\left(C^{\bullet}\right)}=1$. The lemma follows now from (4-5).

The above lemma explains why we call $\rho_{\Gamma}$ the refined torsion: the classical combinatorial torsion of Milnor [25] is an element $\rho$ of $\operatorname{Det}\left(H^{\bullet}(\partial)\right)$, defined up to a multiplication by $t \in \mathbf{k}$ with $|t|=1$, such that $\|\rho\|_{\operatorname{Det}\left(H^{\bullet}(\partial)\right)}=1$. The refined torsion $\rho_{\Gamma}$ is a choice of a particular element of $\operatorname{Det}\left(H^{\bullet}(\partial)\right)$ with norm 1.

\subsection{The refined torsion of a direct sum}

Lemma 4.7 Let $\left(C^{\bullet}, \partial\right)$ and $\left(\widetilde{C}^{\bullet}, \widetilde{\partial}\right)$ be length $d=2 r-1$ complexes of finite dimensional $\mathbf{k}$-vector spaces and let $\Gamma: C^{\bullet} \rightarrow C^{\bullet}, \widetilde{\Gamma}: \widetilde{C}^{\bullet} \rightarrow \widetilde{C}^{\bullet}$ be chirality operators. Then $\hat{\Gamma}:=\Gamma \oplus \widetilde{\Gamma}: C^{\bullet} \oplus \widetilde{C}^{\bullet} \rightarrow C^{\bullet} \oplus \widetilde{C}^{\bullet}$ is a chirality operator on the direct sum complex $\left(C^{\bullet} \oplus \widetilde{C} \bullet, \partial \oplus \widetilde{\partial}\right)$ and

$$
\rho_{\widehat{\Gamma}}=\mu_{H \bullet(\partial), H \bullet(\tilde{\partial})}\left(\rho_{\Gamma} \otimes \rho_{\widetilde{\Gamma}}\right) .
$$

Proof Clearly, $\hat{\Gamma}^{2}=1$ and $\hat{\Gamma}\left(C^{j} \oplus \tilde{C}^{j}\right)=C^{d-j} \oplus \widetilde{C}^{d-j}$. Hence, $\hat{\Gamma}$ is a chirality operator. By Lemma 2.7, to prove (4-6) it is enough to show that

$$
c_{\widehat{\Gamma}}=\mu_{C \cdot, \tilde{C}^{\bullet}}\left(c_{\Gamma} \otimes c_{\widetilde{\Gamma}}\right) .
$$


For each $j=0, \ldots, r-1$, fix nonzero elements $c_{j} \in \operatorname{Det}\left(C^{j}\right), \tilde{c}_{j} \in \widetilde{C}^{j}$ and set $\widehat{c}_{j}=\mu_{C^{j}, \widetilde{C}^{j}}\left(c_{j} \otimes \widetilde{c}_{j}\right)$. Recall that we denoted the operators induced by $\Gamma$ and $\widetilde{\Gamma}$ on $\operatorname{Det}\left(C^{\bullet}\right)$ and $\operatorname{Det}\left(\tilde{C}^{\bullet}\right)$ by the same letters. Thus,

$$
\widehat{\Gamma} \hat{c}_{j}=(\Gamma \oplus \widetilde{\Gamma}) \circ \mu_{C^{j}, \widetilde{C}^{j}}\left(c_{j} \otimes \widetilde{c}_{j}\right)=\mu_{C^{d-j}, \widetilde{C}^{d-j}}\left(\Gamma c_{j} \otimes \widetilde{\Gamma} \widetilde{c}_{j}\right) .
$$

By (2-8),

$$
\mu_{C^{j}, \widetilde{C}^{j}}^{-1}\left(c_{j}^{-1} \otimes \widetilde{c}_{j}^{-1}\right)=\left(\mu_{C^{j}, \widetilde{C}^{j}}\left(c_{j} \otimes \widetilde{c}_{j}\right)\right)^{-1} .
$$

Hence, it follows from (2-18) and (4-1) that

$$
\begin{aligned}
& \mu_{C}, \tilde{C}^{\bullet}\left(c_{\Gamma} \otimes c_{\tilde{\Gamma}}\right)=(-1)^{\mathcal{M}\left(C^{\bullet}, \tilde{C}^{\bullet}\right)+\mathcal{R}\left(C^{\bullet}\right)+\mathcal{R}\left(\tilde{C}^{\bullet}\right)} \times \widehat{c}_{0} \otimes \widehat{c}_{1}^{-1} \otimes \\
& \cdots \otimes \widehat{c}_{r-1}^{(-1)^{r-1}} \otimes \hat{\Gamma}\left(\hat{c}_{r-1}\right)^{(-1)^{r}} \otimes \hat{\Gamma}\left(\widehat{c}_{r-2}\right)^{(-1)^{r+1}} \otimes \cdots \otimes \hat{\Gamma}\left(\hat{c}_{0}\right)^{-1}
\end{aligned}
$$

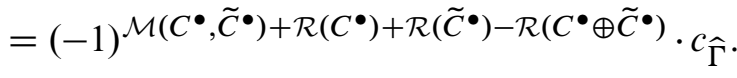

Using the isomorphisms $\Gamma: C^{\bullet} \rightarrow C^{d-\bullet}$ and $\widetilde{\Gamma}: \widetilde{C}^{\bullet} \rightarrow \widetilde{C}^{d-\bullet}$ one sees that $\operatorname{dim} C^{j}=$ $\operatorname{dim} C^{d-j}$ and $\operatorname{dim} \widetilde{C}^{j}=\operatorname{dim} \widetilde{C}^{d-j}$. Therefore,

$$
\begin{aligned}
\mathcal{M}\left(C^{\bullet}, \tilde{C}^{\bullet}\right) & =\sum_{0 \leq k<j \leq d} \operatorname{dim} C^{j} \cdot \operatorname{dim} \tilde{C}^{k} \\
& =\sum_{0 \leq k<j \leq d} \operatorname{dim} C^{d-j} \cdot \operatorname{dim} \widetilde{C}^{d-k}=\sum_{0 \leq k<j \leq d} \operatorname{dim} C^{k} \cdot \operatorname{dim} \widetilde{C}^{j} .
\end{aligned}
$$

Hence,

$$
\begin{aligned}
\mathcal{M}\left(C^{\bullet}, \tilde{C}^{\bullet}\right) & =\frac{1}{2} \sum_{0 \leq k \neq j \leq d} \operatorname{dim} C^{j} \cdot \operatorname{dim} \tilde{C}^{k} \\
& =\frac{1}{2}\left[\left(\sum_{j=0}^{d} \operatorname{dim} C^{j}\right) \cdot\left(\sum_{j=0}^{d} \operatorname{dim} \tilde{C}^{j}\right)-\sum_{j=0}^{d} \operatorname{dim} C^{j} \cdot \operatorname{dim} \tilde{C}^{j}\right] \\
& =\frac{1}{2}\left[\left(2 \sum_{j=0}^{r-1} \operatorname{dim} C^{j}\right) \cdot\left(2 \sum_{j=0}^{r-1} \operatorname{dim} \widetilde{C}^{j}\right)-2 \sum_{j=0}^{r-1} \operatorname{dim} C^{j} \cdot \operatorname{dim} \tilde{C}^{j}\right] \\
& \equiv \sum_{j=0}^{r-1} \operatorname{dim} C^{j} \cdot \operatorname{dim} \widetilde{C}^{j} \bmod 2 .
\end{aligned}
$$

Using the identity (2-30), we obtain from (4-2)

$$
\mathcal{R}\left(C^{\bullet} \oplus \tilde{C}^{\bullet}\right)-\mathcal{R}\left(C^{\bullet}\right)-\mathcal{R}\left(\tilde{C}^{\bullet}\right)=\sum_{j=0}^{r-1} \operatorname{dim} C^{j} \cdot \operatorname{dim} \widetilde{C}^{j} .
$$


Combining (4-9) and (4-10), we get

$$
\mathcal{M}\left(C^{\bullet}, \widetilde{C}^{\bullet}\right)+\mathcal{R}\left(C^{\bullet}\right)+\mathcal{R}\left(\widetilde{C}^{\bullet}\right)-\mathcal{R}\left(C^{\bullet} \oplus \widetilde{C}^{\bullet}\right) \equiv 0 \bmod 2 .
$$

The identity (4-7) follows now from (4-8).

\subsection{Dependence of the refined torsion on the chirality operator}

In this subsection $\mathbf{k}=\mathbb{C}$ or $\mathbb{R}$. Suppose that $\Gamma_{t}, t \in \mathbb{R}$, is a smooth family of chirality operators on the complex $\left(C^{\bullet}, \partial\right)$. Let $\dot{\Gamma}_{t}: C^{\bullet} \rightarrow C^{d-\bullet}$ denote the derivative of $\Gamma_{t}$ with respect to $t$. Then, for each $k=0, \ldots, d$, the composition $\dot{\Gamma}_{t} \circ \Gamma_{t}$ maps $C^{k}$ into itself. In particular $\dot{\Gamma}_{t} \circ \Gamma_{t}: C^{\text {even }} \rightarrow C^{\text {even }}$ and $\dot{\Gamma}_{t} \circ \Gamma_{t}: C^{\text {odd }} \rightarrow C^{\text {odd }}$. Define the supertrace $\operatorname{Tr}_{s}\left(\dot{\Gamma}_{t} \circ \Gamma_{t}\right)$ of $\dot{\Gamma}_{t} \circ \Gamma_{t}$ by the formula

(4-12) $\operatorname{Tr}_{s}\left(\dot{\Gamma}_{t} \circ \Gamma_{t}\right):=\operatorname{Tr}\left(\left.\dot{\Gamma}_{t} \circ \Gamma_{t}\right|_{C^{\text {even }}}\right)-\operatorname{Tr}\left(\left.\dot{\Gamma}_{t} \circ \Gamma_{t}\right|_{C^{\text {odd }}}\right)=\sum_{j=0}^{d}(-1)^{j} \operatorname{Tr}\left(\left.\dot{\Gamma}_{t} \circ \Gamma_{t}\right|_{C^{j}}\right)$.

Proposition 4.9 Let $\left(C^{\bullet}, \partial\right)$ be a length $d=2 r-1$ complex of finite dimensional $\mathbf{k}$-vector spaces and let $\Gamma_{t}, t \in \mathbb{R}$, be a smooth family of chirality operators on $C^{\bullet}$. Then the following equality holds

$$
\frac{d}{d t} \rho_{\Gamma_{t}}=\frac{1}{2} \operatorname{Tr}_{s}\left(\dot{\Gamma}_{t} \circ \Gamma_{t}\right) \cdot \rho_{\Gamma_{t}}
$$

Proof Let $\Gamma_{t, j}$ denote the restriction of $\Gamma_{t}$ to $C^{j}$. Above we denoted the map $\operatorname{Det}\left(C^{j}\right) \rightarrow \operatorname{Det}\left(C^{d-j}\right)$ induced by $\Gamma_{t}$ by the same symbol $\Gamma_{t}$. To avoid confusion we will not use that convention in this proof and denote this map by $\Gamma_{t, j}^{\mathrm{Det}}$.

For each $j=0, \ldots, r-1, t_{0} \in \mathbb{R}$ we have $\Gamma_{t, j}=\Gamma_{t, j} \circ \Gamma_{t_{0}, d-j} \circ \Gamma_{t_{0}, j}$ and, hence,

(4-14) $\left.\frac{d}{d t}\right|_{t=t_{0}} \Gamma_{t, j}^{\mathrm{Det}}=\left.\frac{d}{d t}\right|_{t=t_{0}}\left[\operatorname{Det}\left(\Gamma_{t, j} \circ \Gamma_{t_{0}, d-j}\right) \Gamma_{t_{0}, j}^{\mathrm{Det}}\right]=\operatorname{Tr}\left(\dot{\Gamma}_{t_{0}, j} \circ \Gamma_{t_{0}, d-j}\right) \Gamma_{t_{0}, j}^{\mathrm{Det}}$,

where for the latter equality we used that for any smooth family of operators $A_{t}: C^{d-j} \rightarrow$ $C^{d-j}$ one has $\frac{d}{d t} \operatorname{Det}\left(A_{t}\right)=\operatorname{Tr}\left(\dot{A}_{t} A_{t}^{-1}\right) \cdot \operatorname{Det}\left(A_{t}\right)$ and that $\Gamma_{t, j}^{-1}=\Gamma_{t, d-j}$. Hence, for any nonzero element $c_{j} \in \operatorname{Det}\left(C^{j}\right)$, we have

$$
\frac{d}{d t}\left(\Gamma_{t, j}^{\mathrm{Det}}\left(c_{j}\right)\right)^{ \pm 1}= \pm \operatorname{Tr}\left(\dot{\Gamma}_{t, j} \circ \Gamma_{t, d-j}\right) \cdot\left(\Gamma_{t, j}^{\mathrm{Det}}\left(c_{j}\right)\right)^{ \pm 1}
$$


Using (4-15) and the equality $(-1)^{j+1}=(-1)^{d-j}$ we conclude from the definition (4-1) of the element $c_{\Gamma_{t}}$ that

$$
\frac{d}{d t} c_{\Gamma_{t}}=\sum_{j=0}^{r-1}(-1)^{d-j} \operatorname{Tr}\left(\dot{\Gamma}_{t, j} \circ \Gamma_{t, d-j}\right) \cdot c_{\Gamma_{t}} .
$$

Since $\Gamma_{t, j} \circ \Gamma_{t, d-j}=1$ we obtain

$$
0=\frac{d}{d t} \operatorname{Tr}\left(\Gamma_{t, j} \circ \Gamma_{t, d-j}\right)=\operatorname{Tr}\left(\dot{\Gamma}_{t, j} \circ \Gamma_{t, d-j}\right)+\operatorname{Tr}\left(\Gamma_{t, j} \circ \dot{\Gamma}_{t, d-j}\right) .
$$

Hence,

$$
\operatorname{Tr}\left(\dot{\Gamma}_{t, j} \circ \Gamma_{t, d-j}\right)=-\operatorname{Tr}\left(\dot{\Gamma}_{t, d-j} \circ \Gamma_{t, j}\right) .
$$

Combining (4-16) with (4-17), we obtain (4-13).

\subsection{The refined torsion of the dual complex}

Suppose now that $\mathbf{k}$ is endowed with an involutive endomorphism $\tau$, cf Section 3 . Let $\widehat{C}^{\bullet}$ be the $\tau$-dual complex of $C$ and let $\alpha_{C^{\bullet}}: \operatorname{Det}\left(C^{\bullet}\right) \rightarrow \operatorname{Det}\left(\widehat{C}^{\bullet}\right)$ denote the $\tau$-isomorphism defined in (3-16). Let $\Gamma^{*}$ be the $\tau$-adjoint of $\Gamma$, cf Section 3.3. Then $\widehat{\Gamma}$ is a chirality operator for the complex $\widehat{C}^{\bullet}$.

Lemma 4.11 In the situation described above,

$$
\rho_{\Gamma^{*}}=\alpha_{H^{\bullet}(\partial)}\left(\rho_{\Gamma}\right) .
$$

Proof Fix $c_{j} \in \operatorname{Det}\left(C^{j}\right), j=0, \ldots, r-1$, and set

$$
\widehat{c}_{j}=\alpha_{C^{d-j}}^{-1}\left(\left(\Gamma c_{j}\right)^{-1}\right) \in \operatorname{Det}\left(\widehat{C}^{j}\right), \quad j=0, \ldots, r-1 .
$$

Then, by (3-11),

$$
\Gamma^{*} \widehat{c}_{j}=\alpha_{C^{j}}^{-1}\left(c_{j}^{-1}\right) \in \operatorname{Det}\left(\widehat{C}^{d-j}\right), \quad j=0, \ldots, r-1 .
$$

Using (3-8), we obtain from (4-19) and (4-20), that, for $j=0, \ldots, r-1$,

$$
\beta_{C^{j}}\left(c_{j}\right)=(-1)^{\operatorname{dim} C^{j}} \cdot\left(\Gamma^{*} \widehat{c}_{j}\right)^{-1}, \quad \beta_{C^{d-j}}\left(\Gamma c_{j}\right)=(-1)^{\operatorname{dim} C^{j}} \cdot \widehat{c}_{j}^{-1} .
$$

Combining (3-13), (4-1), (4-19), (4-20), and (4-21), we get

$$
\alpha_{C} \bullet\left(c_{\Gamma}\right)=(-1)^{\mathcal{M}\left(C^{\bullet}\right)+\sum_{p=0}^{r-1} \operatorname{dim} C^{2 p}} \cdot c_{\Gamma^{*}} \cdot
$$

By definition (4-3), $\rho_{\Gamma}=\phi_{C} \bullet\left(c_{\Gamma}\right)$. Therefore, from Lemma 3.6, we obtain

$$
\alpha_{H^{\bullet}(\partial)}\left(\rho_{\Gamma}\right)=\phi_{\hat{C}^{\bullet}} \circ \alpha_{C^{\bullet}}\left(c_{\Gamma}\right)=(-1)^{\mathcal{M}\left(C^{\bullet}\right)+\sum_{p=0}^{r-1} \operatorname{dim} C^{2 p}} \cdot \rho_{\Gamma^{*}} \cdot
$$


Since, by assumption $\Gamma\left(C^{j}\right)=C^{d-j}$, we have $\operatorname{dim} C^{j}=\operatorname{dim} C^{d-j}$. Hence,

$$
\sum_{p=0}^{r-1} \operatorname{dim} C^{2 p}=\frac{1}{2} \sum_{j=0}^{d} \operatorname{dim} C^{j},
$$

and, by (3-14),

$$
\begin{aligned}
\mathcal{M}\left(C^{\bullet}\right)+\sum_{p=0}^{r-1} \operatorname{dim} C^{2 p}= & \sum_{0 \leq j<k \leq d} \operatorname{dim} C^{j} \cdot \operatorname{dim} C^{k}+\frac{1}{2} \sum_{j=0}^{d} \operatorname{dim} C^{j} \\
& =\frac{1}{2} \sum_{j, k=0}^{d} \operatorname{dim} C^{j} \cdot \operatorname{dim} C^{k}=\frac{1}{2}\left(\sum_{j=0}^{d} \operatorname{dim} C^{j}\right)^{2} .
\end{aligned}
$$

Using again the equality $\operatorname{dim} C^{j}=\operatorname{dim} C^{d-j}$, we obtain

$$
\sum_{j=0}^{d} \operatorname{dim} C^{j}=2 \sum_{j=0}^{r-1} \operatorname{dim} C^{j}
$$

Hence, from (4-24) we get

$$
\mathcal{M}\left(C^{\bullet}\right)+\sum_{p=0}^{r-1} \operatorname{dim} C^{2 p}=2\left(\sum_{j=0}^{r-1} \operatorname{dim} C^{j}\right)^{2} \equiv 0, \bmod 2 .
$$

Combining (4-23) with (4-25), we obtain (4-18).

\section{Calculation of the refined torsion of a finite dimensional complex}

In this section we introduce a finite dimensional analogue of the Atiyah-Patodi-Singer odd signature operator and express the refined torsion in terms of the determinant of this operator.

Throughout the section we work under the assumptions of Section 4.1.

\subsection{The signature operator}

The signature operator $\mathcal{B}$ is defined by the formula

$$
\mathcal{B}:=\Gamma \partial+\partial \Gamma .
$$


This is a direct analogue of the signature operator of an odd-dimensional manifold, cf [1, page 44], [2, page 405], [19, pages 64-65]. See also Section 7 below.

For $j=0, \ldots, d$ define

$$
\begin{aligned}
& C_{+}^{j}:=\operatorname{Ker}(\partial \circ \Gamma) \cap C^{j}=\Gamma\left(\operatorname{Ker} \partial \cap C^{d-j}\right), \\
& C_{-}^{j}:=\operatorname{Ker} \partial \cap C^{j}
\end{aligned}
$$

and set $C_{+}^{-1}=C_{-}^{d+1}=0$. Let $\mathcal{B}_{j}$ and $\mathcal{B}_{j}^{ \pm}$denote the restriction of $\mathcal{B}$ to $C^{j}$ and $C_{ \pm}^{j}$ respectively. Then, for each $j=0, \ldots, d$, one has

$$
\begin{aligned}
& \operatorname{Im} \mathcal{B}_{j}^{+} \subseteq \operatorname{Im}\left(\left.\Gamma \circ \partial\right|_{C^{j}}\right) \subseteq \Gamma\left(\left.\operatorname{Ker} \partial\right|_{C^{j+1}}\right) \subseteq C_{+}^{d-j-1} \\
& \operatorname{Im} \mathcal{B}_{j}^{-} \subseteq \operatorname{Im}\left(\left.\partial \circ \Gamma\right|_{C^{j}}\right) \subseteq \operatorname{Im}\left(\left.\partial\right|_{C^{d-j}}\right) \subseteq C_{-}^{d-j+1}
\end{aligned}
$$

Hence,

$$
\mathcal{B}_{j}^{+}=\Gamma \circ \partial: C_{+}^{j} \longrightarrow C_{+}^{d-j-1}, \quad \mathcal{B}_{j}^{-}=\partial \circ \Gamma: C_{-}^{j} \longrightarrow C_{-}^{d-j+1} .
$$

Denote $C^{\text {even }}:=\bigoplus_{j \text { even }} C^{j}, C_{ \pm}^{\text {even }}:=\bigoplus_{j \text { even }} C_{ \pm}^{j}$ and set

$$
\begin{aligned}
& \mathcal{B}_{\text {even }}:=\bigoplus_{j \text { even }} \mathcal{B}_{j}: C^{\text {even }} \rightarrow C^{\text {even }}, \\
& \mathcal{B}_{\text {even }}^{ \pm}:=\bigoplus_{j \text { even }} \mathcal{B}_{j}^{ \pm}: C_{ \pm}^{\text {even }} \rightarrow C_{ \pm}^{\text {even }},
\end{aligned}
$$

and define $\mathcal{B}_{\text {odd }}, \mathcal{B}_{\text {odd }}^{ \pm}$similarly. Note that $\mathcal{B}_{\text {even }}=\Gamma \circ \mathcal{B}_{\text {odd }} \circ \Gamma$. Hence, the whole information about $\mathcal{B}$ is encoded in its even part $\mathcal{B}_{\text {even }}$.

Lemma 5.2 Suppose that the signature operator $\mathcal{B}: C^{\bullet} \rightarrow C^{\bullet}$ is bijective. Then the complex $\left(C^{\bullet}, \partial\right)$ is acyclic and, for all $j=0, \ldots, d$,

$$
C^{j}=C_{+}^{j} \oplus C_{-}^{j} .
$$

Proof If $c \in C_{+}^{j} \cap C_{-}^{j}$, then it follows from (5-2) and the definition (5-1) of $\mathcal{B}$ that $\mathcal{B} c=0$. Hence, since $\mathcal{B}$ is injective, we obtain

$$
C_{+}^{j} \cap C_{-}^{j}=\{0\} .
$$

Similarly, from (5-2) and (5-1) we obtain $\operatorname{Im} \mathcal{B} \subset C_{+}^{\bullet}+C_{-}^{\bullet}$. Hence, since $\mathcal{B}$ is surjective,

$$
C^{\bullet}=C_{+}^{\bullet}+C_{-}^{\bullet} .
$$

Combining (5-8) and (5-9) we obtain (5-7). 
Let us now show that the complex $\left(C^{\bullet}, \partial\right)$ is acyclic. By $(5-7)$,

$$
\mathcal{B}=\mathcal{B}^{+}+\mathcal{B}^{-} \text {. }
$$

By (5-3) and (5-4), $\operatorname{Im} \mathcal{B}^{ \pm} \subset C_{ \pm}^{\bullet}$. Thus, since $\mathcal{B}=\mathcal{B}^{+}+\mathcal{B}^{-}$is surjective, it follows from (5-7), that $\operatorname{Im} \mathcal{B}^{ \pm}=C_{ \pm}^{\bullet}$. The equality $\mathcal{B}^{-}=\partial \circ \Gamma$ implies now that $\operatorname{Im} \partial \supset C_{-}^{\bullet}$. Hence, by (5-2), $\operatorname{Im} \partial=C_{-}^{\bullet}$. Combining the latter equality with the definition (5-2) of $C_{-}^{\bullet}$, we obtain $\operatorname{Im} \partial=\operatorname{Ker} \partial$, proving the acyclicity of $\left(C^{\bullet}, \partial\right)$.

Remark 5.3 It is easy to construct an acyclic complex $\left(C^{\bullet}, \partial\right)$ and a chirality operator $\Gamma$, so that the corresponding signature operator $\mathcal{B}$ is not bijective.

\subsection{Calculation of the refined torsion in case $\mathcal{B}$ is bijective}

Assume that the signature operator $\mathcal{B}: C^{\bullet} \rightarrow C^{\bullet}$ is bijective. Then, by Lemma 5.2, the complex $\left(C^{\bullet}, \partial\right)$ is acyclic. Hence, $\operatorname{Det}\left(H^{\bullet}(\partial)\right)$ is canonically isomorphic to $\mathbf{k}$ and the refined torsion $\rho_{\Gamma}$ can be viewed as a number in $\mathbf{k}$. In this subsection we calculate this number.

Definition 5.5 The graded determinant of the even part of the signature operator is defined by the formula

$$
\operatorname{Det}_{\text {gr }}\left(\mathcal{B}_{\text {even }}\right)=\operatorname{Det}\left(\mathcal{B}_{\text {even }}^{+}\right) / \operatorname{Det}\left(-\mathcal{B}_{\text {even }}^{-}\right)
$$

Since $\Gamma \circ \mathcal{B}_{\text {even }}^{-} \circ \Gamma=\mathcal{B}_{\text {odd }}^{+}$and $\Gamma^{2}=\mathrm{Id}$, we have $\operatorname{Det}\left(-\mathcal{B}_{\text {even }}^{-}\right)=\operatorname{Det}\left(-\mathcal{B}_{\text {odd }}^{+}\right)$and

$$
\begin{aligned}
& \operatorname{Det}{ }_{\mathrm{gr}}\left(\mathcal{B}_{\mathrm{even}}\right)=\operatorname{Det}\left(\mathcal{B}_{\mathrm{even}}^{+}\right) / \operatorname{Det}\left(-\mathcal{B}_{\text {odd }}^{+}\right) \\
& =\operatorname{Det}\left(\left.(-1)^{r-1} \Gamma \partial\right|_{C_{+}^{r-1}}\right)^{(-1)^{r-1}} \prod_{j=1}^{r-1} \operatorname{Det}\left(\left.(-1)^{j-1} \Gamma \partial\right|_{\left.C_{+}^{j-1} \oplus C_{+}^{d-j}\right)^{(-1)^{j-1}}}\right. \\
& =(-1)^{(r-1) \operatorname{dim} C_{+}^{r-1}} \operatorname{Det}\left(\left.\Gamma \partial\right|_{C_{+}^{r-1}}\right)^{(-1)^{r-1}} \prod_{j=1}^{r-1} \operatorname{Det}\left(\left.\Gamma \partial\right|_{C_{+}^{j-1} \oplus C_{+}^{d-j}}\right)^{(-1)^{j-1}},
\end{aligned}
$$

where in the last equality we used that

$$
\operatorname{Det}\left(\left.(-1)^{j-1} \Gamma \partial\right|_{C_{+}^{j-1} \oplus C_{+}^{d-j}}\right)=\operatorname{Det}\left(\left.\Gamma \partial\right|_{C_{+}^{j-1} \oplus C_{+}^{d-j}}\right)
$$

since $\operatorname{dim} C_{+}^{j-1}=\operatorname{dim} C_{+}^{d-j}$.

Proposition 5.6 Suppose that the signature operator $\mathcal{B}$ is invertible and, hence, the complex $\left(C^{\bullet}, \partial\right)$ is acyclic. Then

$$
\rho_{\Gamma}=\operatorname{Det}_{\text {gr }}\left(\mathcal{B}_{\text {even }}\right) .
$$


Proof Recall that $\rho_{\Gamma}=\phi_{C} \bullet\left(c_{\Gamma}\right)$, where $c_{\Gamma}$ is the element in $\operatorname{Det}\left(C^{\bullet}\right)$ given by the formula (4-1) and

$$
\phi_{C^{\bullet}: \operatorname{Det}}\left(C^{\bullet}\right) \longrightarrow \operatorname{Det}\left(H^{\bullet}(\partial)\right) \simeq \mathbf{k}
$$

is defined by (2-13).

To compute $\phi_{C} \bullet\left(c_{\Gamma}\right)$, we choose the decomposition (2-11) to be $C^{j}=C_{-}^{j} \oplus C_{+}^{j}$ and define elements $c_{0}, \ldots, c_{d}$ as follows: For each $j=0, \ldots, d-1$, fix a nonzero element $a_{j} \in \operatorname{Det}\left(C_{+}^{j}\right)$ and set

$$
c_{0}=a_{0}, \quad c_{d}=\Gamma a_{0}, \quad c_{j}=\mu_{C^{j}, C_{+}^{j}}\left(\Gamma a_{d-j} \otimes a_{j}\right), \quad j=1, \ldots, d-1 .
$$

Note that, for each $j=1, \ldots, d$,

$$
\begin{aligned}
\Gamma c_{j} & =\mu_{C_{+}^{d-j}, C_{-}^{d-j}}\left(a_{d-j} \otimes \Gamma a_{j}\right) \\
& =(-1)^{\operatorname{dim} C_{+}^{j} \cdot \operatorname{dim} C_{-}^{j}} \mu_{C_{-}^{d-j}, C_{+}^{d-j}}\left(\Gamma a_{j} \otimes a_{d-j}\right)=(-1)^{\operatorname{dim} C_{+}^{j} \cdot \operatorname{dim} C_{-}^{j}} c_{d-j} .
\end{aligned}
$$

Thus, from (4-1), we obtain

$$
\begin{aligned}
c_{\Gamma}=(-1)^{\mathcal{R}\left(C^{\bullet}\right)} \cdot c_{0} \otimes c_{1}^{-1} \otimes \cdots c_{r-1}^{(-1)^{r-1}} \\
\\
\quad \otimes\left(\Gamma c_{r-1}\right)^{(-1)^{r}} \otimes\left(\Gamma c_{r-2}\right)^{(-1)^{r-1}} \otimes \cdots \otimes\left(\Gamma c_{0}\right)^{-1} \\
=(-1)^{\mathcal{R}\left(C^{\bullet}\right)+\sum_{j=1}^{r-1} \operatorname{dim} C_{+}^{j} \cdot \operatorname{dim} C_{-}^{j}} c_{0} \wedge c_{1}^{-1} \wedge \cdots \wedge c_{d-1} \wedge c_{d}^{-1} .
\end{aligned}
$$

To compute $\rho_{\Gamma}$ we now need to calculate $\phi_{C} \bullet\left(c_{0} \wedge c_{1}^{-1} \wedge \cdots \wedge c_{d-1} \wedge c_{d}^{-1}\right)$, ie, in view of (2-14), we need to determine the elements $h_{j} \in \operatorname{Det}\left(H^{j}\right) \simeq \mathbf{k}$ which satisfy (2-12).

If $L$ is a $\mathbf{k}$-line and $x, y \in L$ with $y \neq 0$, we denote by $[x: y] \in \mathbf{k}$ the unique number such that $x=[x: y] y$. Then, by (2-12), the elements $h_{j} \in \mathbf{k}$, which appear in (2-14), are given by

$$
\begin{aligned}
& h_{0}=1, \\
& h_{d}=\left[c_{d}: \partial a_{d-1}\right]=\left[a_{0}: \Gamma \partial a_{d-1}\right],
\end{aligned}
$$

and, for $j=1, \ldots, d-1$,

$$
\begin{aligned}
h_{j} & =\left[c_{j}: \mu_{C \underline{j}, C_{+}^{j}}\left(\partial a_{j-1} \otimes a_{j}\right)\right] \\
& =\left[\mu_{C_{-}^{j}, C_{+}^{j}}\left(\Gamma a_{d-j} \otimes a_{j}\right): \mu_{C_{-}^{j}, C_{+}^{j}}\left(\partial a_{j-1} \otimes a_{j}\right)\right] \\
& =\left[\Gamma a_{d-j}: \partial a_{j-1}\right]=\left[a_{d-j}: \Gamma \partial a_{j-1}\right] .
\end{aligned}
$$


By (5-17) and (5-18), for $j=1, \ldots, r-1$, we obtain

$$
\begin{aligned}
& h_{j} \cdot h_{d-j+1}=\left[a_{d-j}: \Gamma \partial a_{j-1}\right] \cdot\left[a_{j-1}: \Gamma \partial a_{d-j}\right] \\
& =\left[\mu_{C_{+}^{d-j}, C_{+}^{j-1}}\left(a_{d-j} \otimes a_{j-1}\right): \mu_{C_{+}^{d-j}, C_{+}^{j-1}}\left(\Gamma \partial a_{j-1} \otimes \Gamma \partial a_{d-j}\right]\right. \\
& =(-1)^{\operatorname{dim} C_{+}^{d-j} \cdot \operatorname{dim} C_{+}^{j-1}} \operatorname{Det}\left(\left.\Gamma \partial\right|_{C_{+}^{j-1} \oplus C_{+}^{d-j}}\right)^{-1} \text {. }
\end{aligned}
$$

By (5-18) we have

$$
h_{r}=\operatorname{Det}\left(\left.\Gamma \partial\right|_{C_{+}^{r-1}}\right)^{-1} .
$$

Combining (2-14), (5-16), (5-19), and (5-20), we obtain

$$
\begin{aligned}
\phi_{C} \bullet\left(c_{0}\right. & \left.\wedge c_{1}^{-1} \wedge \cdots \wedge c_{d-1} \wedge c_{d}^{-1}\right) \\
= & (-1)^{\mathcal{N}\left(C^{\bullet}\right)} \operatorname{Det}\left(\left.\Gamma \partial\right|_{C^{r-1}}\right)^{(-1)^{r-1}} \cdot \prod_{j=1}^{r-1} \operatorname{Det}\left(\left.\Gamma \partial\right|_{C_{+}^{j-1} \oplus C_{+}^{d-j}}\right)^{(-1)^{j-1}}
\end{aligned}
$$

From the definition (4-3) of $\rho_{\Gamma}$ and the identities (5-15), (5-21), we get

$$
\rho_{\Gamma}=(-1)^{\mathcal{F}\left(C^{\bullet}\right)} \operatorname{Det}\left(\left.\Gamma \partial\right|_{C^{r-1}}\right)^{(-1)^{r-1}} \cdot \prod_{j=1}^{r-1} \operatorname{Det}\left(\left.\Gamma \partial\right|_{C_{+}^{j-1} \oplus C_{+}^{d-j}}\right)^{(-1)^{j-1}}
$$

where

$$
\mathcal{F}\left(C^{\bullet}\right)=\mathcal{N}\left(C^{\bullet}\right)+\mathcal{R}\left(C^{\bullet}\right)+\sum_{j=0}^{r-1} \operatorname{dim} C_{+}^{j} \cdot \operatorname{dim} C_{-}^{j}+\sum_{j=0}^{r-1} \operatorname{dim} C_{+}^{d-j} \cdot \operatorname{dim} C_{+}^{j-1}
$$

As the maps $\partial: C_{+}^{j-1} \rightarrow C_{-}^{j}$ and $\Gamma: C_{+}^{d-j} \rightarrow C_{-}^{j}$ are isomorphisms, the last two terms in (5-23) can be computed to be

$$
\begin{aligned}
& \sum_{j=0}^{r-1} \operatorname{dim} C_{+}^{j} \cdot \operatorname{dim} C_{-}^{j}+\sum_{j=0}^{r-1} \operatorname{dim} C_{+}^{d-j} \cdot \operatorname{dim} C_{+}^{j-1} \\
= & \sum_{j=0}^{r-1} \operatorname{dim} C_{+}^{j} \cdot \operatorname{dim} C_{+}^{j-1}+\sum_{j=0}^{r-1} \operatorname{dim} C_{-}^{j} \cdot \operatorname{dim} C_{+}^{j-1}=\sum_{j=0}^{r-1} \operatorname{dim} C^{j} \cdot \operatorname{dim} C_{+}^{j-1} .
\end{aligned}
$$

Since the map $\Gamma \partial: C_{+}^{j} \rightarrow C_{+}^{d-j-1}$ is an isomorphism, for all $j=0, \ldots, r-2$ we have

$$
\operatorname{dim} C_{+}^{j} \cdot\left(\operatorname{dim} C_{+}^{j}+(-1)^{j+1}\right)=\operatorname{dim} C_{+}^{d-j-1} \cdot\left(\operatorname{dim} C_{+}^{d-j-1}+(-1)^{d-j}\right) .
$$


Hence, by formula (2-15) for $\mathcal{N}(C)$ and the fact that for any $x \in \mathbb{Z}, x(x \pm 1) \equiv$ $0(\bmod 2)$, we obtain

$$
\begin{aligned}
\mathcal{N}\left(C^{\bullet}\right) & =\sum_{j=0}^{r-2} \operatorname{dim} C_{+}^{j} \cdot\left(\operatorname{dim} C_{+}^{j}+(-1)^{j+1}\right)+\frac{1}{2} \operatorname{dim} C_{+}^{r-1} \cdot\left(\operatorname{dim} C_{+}^{r-1}+(-1)^{r}\right) \\
& \equiv \frac{1}{2} \operatorname{dim} C_{+}^{r-1} \cdot\left(\operatorname{dim} C_{+}^{r-1}+(-1)^{r}\right) \\
& =\frac{1}{2} \operatorname{dim} C_{+}^{r-1} \cdot\left(\operatorname{dim} C_{+}^{r-1}-1\right)+\frac{1}{2}\left(1+(-1)^{r}\right) \cdot \operatorname{dim} C_{+}^{r-1} \bmod 2 .
\end{aligned}
$$

Next, using the isomorphism $\partial: C_{+}^{j-1} \rightarrow C_{-}^{j}$ we obtain from (5-7),

$$
\operatorname{dim} C^{j}=\operatorname{dim} C_{+}^{j-1}+\operatorname{dim} C_{+}^{j} .
$$

Hence, from definition (4-2) of $\mathcal{R}\left(C^{\bullet}\right)$ and from identity (2-30), we get

$$
\begin{aligned}
\mathcal{R}\left(C^{\bullet}\right)= & \sum_{j=0}^{r-1}\left[\frac{1}{2} \operatorname{dim} C_{+}^{j} \cdot\left(\operatorname{dim} C_{+}^{j}+(-1)^{r+j}\right)\right. \\
& \left.\quad+\frac{1}{2} \operatorname{dim} C_{+}^{j-1} \cdot\left(\operatorname{dim} C_{+}^{j-1}+(-1)^{r+j-1}\right)+\operatorname{dim} C_{+}^{j} \cdot \operatorname{dim} C_{+}^{j-1}\right] \\
= & \frac{1}{2} \operatorname{dim} C_{+}^{r-1} \cdot\left(\operatorname{dim} C_{+}^{r-1}-1\right)+\sum_{j=0}^{r-2}\left(\operatorname{dim} C_{+}^{j}\right)^{2}+\sum_{j=0}^{r-1} \operatorname{dim} C_{+}^{j} \cdot \operatorname{dim} C_{+}^{j-1} .
\end{aligned}
$$

By (5-26),

$$
\begin{aligned}
& \sum_{j=0}^{r-2}\left(\operatorname{dim} C_{+}^{j}\right)^{2}+\sum_{j=0}^{r-1} \operatorname{dim} C_{+}^{j} \cdot \operatorname{dim} C_{+}^{j-1} \\
& \quad=\sum_{j=1}^{r-1}\left[\left(\operatorname{dim} C_{+}^{j-1}\right)^{2}+\operatorname{dim} C_{+}^{j} \cdot \operatorname{dim} C_{+}^{j-1}\right] \equiv \sum_{j=0}^{r-1} \operatorname{dim} C^{j} \cdot \operatorname{dim} C_{+}^{j-1}
\end{aligned}
$$

Hence, from (5-27), we get

(5-29) $\mathcal{R}\left(C^{\bullet}\right) \equiv \frac{1}{2} \operatorname{dim} C_{+}^{r-1} \cdot\left(\operatorname{dim} C_{+}^{r-1}-1\right)+\sum_{j=0}^{r-1} \operatorname{dim} C^{j} \cdot \operatorname{dim} C_{+}^{j-1}, \quad \bmod 2$.

Combining (5-25) and (5-29) and using again that $x(x \pm 1) \equiv 0$ for $x \in \mathbb{Z}$, we conclude that

$(5-30) \mathcal{N}\left(C^{\bullet}\right)+\mathcal{R}\left(C^{\bullet}\right) \equiv \frac{1}{2}\left(1+(-1)^{r}\right) \operatorname{dim} C_{+}^{r-1}+\sum_{j=0}^{r-1} \operatorname{dim} C^{j} \cdot \operatorname{dim} C_{+}^{j-1}, \bmod 2$ 
Since $\frac{1}{2}\left(1+(-1)^{r}\right) \equiv r-1$ modulo 2, we conclude from (5-23), (5-24), and (5-30), that

$$
\mathcal{F}\left(C^{\bullet}\right) \equiv(r-1) \operatorname{dim} C_{+}^{r-1}, \bmod 2 .
$$

The equality (5-13) follows now from (5-12) and (5-22).

\subsection{Calculation of the refined torsion in case $\mathcal{B}$ is not bijective}

In this section we don't assume that $\mathcal{B}$ is bijective. In particular, the complex $\left(C^{\bullet}, \partial\right)$ is not necessarily acyclic. For simplicity, we restrict to the case $\mathbf{k}=\mathbb{C}$.

Consider the operator $\mathcal{B}^{2}$. Note that $\mathcal{B}^{2}=(\Gamma \partial)^{2}+(\partial \Gamma)^{2}$ and $\mathcal{B}^{2}\left(C^{j}\right) \subset C^{j}$ for all $j=0, \ldots, d$. For an arbitrary interval $\mathcal{I} \subset[0, \infty)$ and $j=0, \ldots, d$, we denote by $C_{\mathcal{I}}^{j} \subset C^{j}$ the span of the generalized eigenvectors of the restriction of $\mathcal{B}^{2}$ to $C^{j}$ corresponding to eigenvalues $\lambda$ with $|\lambda| \in \mathcal{I}$. Since both operators $\Gamma$ and $\partial$ commute with $\mathcal{B}$ and, hence, with $\mathcal{B}^{2}$ we have

$$
\Gamma: C_{\mathcal{I}}^{j} \longrightarrow C_{\mathcal{I}}^{d-j}, \quad \partial: C_{\mathcal{I}}^{j} \longrightarrow C_{\mathcal{I}}^{j+1} .
$$

Hence, we obtain a subcomplex $C_{\mathcal{I}}^{\bullet}$ of $C^{\bullet}$ and the restriction $\Gamma_{\mathcal{I}}$ of $\Gamma$ to $C_{\mathcal{I}}^{\bullet}$ is a chirality operator on this complex. Let $\partial_{\mathcal{I}}, \mathcal{B}_{\mathcal{I}}$, and $\mathcal{B}_{\mathcal{I}}^{\text {even }}$ denote the restriction of $\partial$, $\mathcal{B}$, and $\mathcal{B}_{\text {even }}$ to $C_{\mathcal{I}}^{\bullet}$. Then $\mathcal{B}_{\mathcal{I}}=\Gamma_{\mathcal{I}} \partial_{\mathcal{I}}+\partial_{\mathcal{I}} \Gamma_{\mathcal{I}}$

Lemma 5.8 If $0 \notin \mathcal{I}$ then the complex $\left(C_{\mathcal{I}}^{\bullet}, \partial_{\mathcal{I}}\right)$ is acyclic.

Proof If $x \in \operatorname{Ker} \partial_{\mathcal{I}}$ then $\mathcal{B}^{2} x=(\partial \Gamma)^{2} x \in \operatorname{Im} \partial_{\mathcal{I}}$. Hence,

$$
\mathcal{B}_{\mathcal{I}}^{2}: \operatorname{Ker} \partial_{\mathcal{I}} \longrightarrow \operatorname{Im} \partial_{\mathcal{I}} \subset \operatorname{Ker} \partial_{\mathcal{I}} .
$$

Since the operator $\mathcal{B}_{\mathcal{I}}^{2}: C_{\mathcal{I}}^{\bullet} \rightarrow C_{\mathcal{I}}^{\bullet}$ is invertible, we conclude that $\operatorname{Ker} \partial_{\mathcal{I}}=\operatorname{Im} \partial_{\mathcal{I}}$.

For each $\lambda \geq 0$, the complex $C^{\bullet}$ is a direct sum of the complex $C_{[0, \lambda]}^{\bullet}$ and the acyclic complex $C_{(\lambda, \infty)}^{\bullet}$. In particular, $H_{(\lambda, \infty)}^{\bullet}(\partial)=0$ and $H_{[0, \lambda]}^{\bullet}(\partial) \simeq H^{\bullet}(\partial)$. Hence, there are canonical isomorphisms

$$
\Phi: \operatorname{Det}\left(H_{(\lambda, \infty)}^{\bullet}(\partial)\right) \longrightarrow \mathbb{C}, \quad \Psi: \operatorname{Det}\left(H_{[0, \lambda]}^{\bullet}(\partial)\right) \longrightarrow \operatorname{Det}\left(H^{\bullet}(\partial)\right)
$$

Lemma 5.9 For every $t \in \operatorname{Det}\left(H_{(\lambda, \infty)}^{\bullet}(\partial)\right), h \in \operatorname{Det}\left(H_{[0, \lambda]}^{\bullet}(\partial)\right)$

$$
\Phi(t) \cdot \Psi(h)=\mu_{H_{(\lambda, \infty)}^{\bullet}(\partial), H_{[0, \lambda]}^{\bullet}(\partial)}(t \otimes h) .
$$


Proof Since $H_{(\lambda, \infty)}^{\bullet}(\partial)=0$, it follows from $(2-19)$, that

$$
\mathcal{M}\left(H_{(\lambda, \infty)}^{\bullet}(\partial), H_{[0, \lambda]}^{\bullet}\right)=0 .
$$

The lemma follows now from the definition (2-18) of the fusion isomorphism.

In the sequel we will not distinguish between $t \in \operatorname{Det}\left(H_{(\lambda, \infty)}^{\bullet}(\partial)\right)$ and $\Phi(t) \in \mathbb{C}$ and write simply $t$ for $\Phi(t)$. Similarly, for $h \in \operatorname{Det}\left(H_{[0, \lambda]}^{\bullet}(\partial)\right)$ we will denote by $h$ also the element $\Psi(h) \in \operatorname{Det}\left(H^{\bullet}(\partial)\right)$.

From Lemma 4.7, Proposition 5.6, and Lemma 5.9, we immediately obtain the following Proposition.

Proposition 5.10 Let $\left(C^{\bullet}, \partial\right)$ be a complex of finite dimensional complex vector spaces and let $\Gamma$ be a chirality operator on $C^{\bullet}$. Then, for each $\lambda \geq 0$,

$$
\rho_{\Gamma}=\operatorname{Det}_{\mathrm{gr}}\left(\mathcal{B}_{(\lambda, \infty)}^{\text {even }}\right) \cdot \rho_{\Gamma_{[0, \lambda]}},
$$

where, as above, we view $\rho_{\Gamma_{[0, \lambda]}}$ as an element of $\operatorname{Det}\left(H^{\bullet}(\partial)\right)$ via the canonical isomorphism $\Psi: \operatorname{Det}\left(H_{[0, \lambda]}^{\bullet}(\partial)\right) \rightarrow \operatorname{Det}\left(H^{\bullet}(\partial)\right)$.

\section{Preliminaries on determinants and the $\eta$-invariant of ellip- tic operators}

In this section we briefly review the main facts about the $\zeta$-regularized determinants and $\eta$-invariants of non self-adjoint elliptic operators. In particular, we define a signrefined version of the graded determinant - a notion, which plays a central role in this paper. We refer the reader to of [9, Sections 3 and 4] for a more detailed discussion of the subject.

Let $E$ be a complex vector bundle over a smooth compact manifold $M$ and let $D: C^{\infty}(M, E) \rightarrow C^{\infty}(M, E)$ be an elliptic differential operator of order $m \geq 1$. Denote by $\sigma(D)$ the leading symbol of $D$.

\subsection{Choice of an angle}

Our aim is to define the $\zeta$-function and the determinant of $D$. For this we will need to define the complex powers of $D$. As usual, to define complex powers we need to choose a spectral cut in the complex plane. We restrict ourselves to the spectral cuts given by a ray

$$
R_{\theta}=\left\{\rho e^{i \theta}: 0 \leq \rho<\infty\right\}, \quad 0 \leq \theta<2 \pi .
$$

Consequently, we have to choose an angle $\theta \in[0,2 \pi)$. 
Definition 6.2 The angle $\theta$ is a principal angle for an elliptic operator $D$ if

$$
\operatorname{spec}(\sigma(D)(x, \xi)) \cap R_{\theta}=\varnothing, \quad \text { for all } x \in M, \xi \in T_{x}^{*} M \backslash\{0\} .
$$

If $\mathcal{I} \subset \mathbb{R}$ we denote by $L_{\mathcal{I}}$ the solid angle

$$
L_{\mathcal{I}}=\left\{\rho e^{i \theta}: 0<\rho<\infty, \theta \in \mathcal{I}\right\} .
$$

The existence of a principal angle is an additional assumption on $D$. Since $M$ is compact every operator which possesses a principal angle has a discrete spectrum.

Definition 6.3 The angle $\theta$ is an Agmon angle ${ }^{1}$ for an elliptic operator $D$ if it is a principal angle for $D$ and there exists $\varepsilon>0$ such that

$$
\operatorname{spec}(D) \cap L_{[\theta-\varepsilon, \theta+\varepsilon]}=\varnothing .
$$

If $\theta$ is a principal angle for $D$, then, cf Seeley [31] or Shubin [32], there exists $\varepsilon>0$ such that $\operatorname{spec}(D) \cap L_{[\theta-\varepsilon, \theta+\varepsilon]}$ is finite and $\operatorname{spec}(\sigma(D)) \cap L_{[\theta-\varepsilon, \theta+\varepsilon]}=\varnothing$. Hence there exists an Agmon angle $\theta^{\prime} \in(\theta-\varepsilon, \theta+\varepsilon)$ for $D$.

\section{$6.4 \zeta$-function and determinant}

Assume that $\theta$ is an Agmon angle for $D$. Let $\Pi: L^{2}(M, E) \rightarrow L^{2}(M, E)$ denote the spectral projection of $D$ corresponding to all nonzero eigenvalues of $D$. The $\zeta$-function $\zeta_{\theta}(s, D)$ of $D$ is defined as follows.

Since, by assumption, $D$ possesses a principal angle, its spectrum is discrete. Hence, there exists a small number $\rho_{0}>0$ such that

$$
\operatorname{spec}(D) \cap\left\{z \in \mathbb{C} ;|z|<2 \rho_{0}\right\} \subseteq\{0\} .
$$

Define the contour $\Gamma=\Gamma_{\theta, \rho_{0}} \subset \mathbb{C}$ consisting of three curves $\Gamma=\Gamma_{1} \cup \Gamma_{2} \cup \Gamma_{3}$, where

$$
\begin{aligned}
& \Gamma_{1}=\left\{\rho e^{i \theta}: \infty>\rho \geq \rho_{0}\right\}, \\
& \Gamma_{2}=\left\{\rho_{0} e^{i \alpha}: \theta<\alpha<\theta+2 \pi\right\}, \\
& \Gamma_{3}=\left\{\rho e^{i(\theta+2 \pi)}: \rho_{0} \leq \rho<\infty\right\} .
\end{aligned}
$$

For $\operatorname{Re} s>\frac{\operatorname{dim} M}{m}$, the operator

$$
\Pi D_{\theta}^{-s}=\frac{i}{2 \pi} \int_{\Gamma_{\theta, \rho_{0}}} \lambda^{-s}(D-\lambda)^{-1} d \lambda
$$

\footnotetext{
${ }^{1}$ Note that in the literature the notion of Agmon angle is often defined differently, namely it is required, in addition, that zero is nor in the spectrum of the operator.
} 
is a pseudo-differential operator with continuous kernel $K_{\theta}(s ; x, y)$, cf $[31 ; 32]$. In particular, the operator $\Pi D_{\theta}^{-s}$ is of trace class.

We define

$$
\zeta_{\theta}(s, D)=\operatorname{Tr} \Pi D_{\theta}^{-s}=\int_{M} \operatorname{tr} K_{\theta}(s ; x, x) d x, \quad \operatorname{Re} s>\frac{\operatorname{dim} M}{m} .
$$

It was shown by Seeley [31] (see also [32]) that $\zeta_{\theta}(s, D)$ has a meromorphic extension to the whole complex plane and that 0 is a regular value of $\zeta_{\theta}(s, D)$.

More generally, let $Q$ be a pseudo-differential operator of order $q$. We set

$$
\zeta_{\theta}(s, Q, D)=\operatorname{Tr} Q \Pi D_{\theta}^{-s}, \quad \operatorname{Re} s>(q+\operatorname{dim} M) / m .
$$

This function also has a meromorphic extension to the whole complex plane, see Wodzicki [40, Section 3.22] and Grubb-Seeley [20, Theorem 2.7] (see also Guillemin [21]). Moreover, if $Q$ is a 0 th order pseudo-differential projection, ie a 0 th order pseudo-differential operator satisfying $Q^{2}=Q$, then by Wodzicki [39, Section 7], [40] (see also Brüning-Lesch [10] or Ponge [27] for a shorter proof), $\zeta_{\theta}(s, Q, D)$ is regular at 0 .

If the dimension of $M$ is odd and $D$ is a bijective differential operator of even order, then $\zeta_{\theta}(0, D)=0$, cf Seeley [31]. More generally, we have the following

Proposition 6.5 Suppose $\operatorname{dim} M$ is odd, $D: C^{\infty}(M, E) \rightarrow C^{\infty}(M, E)$ is an elliptic differential operator of even order $m \geq 2, \theta$ is an Agmon angle for $D$, and $P$ is a finite rank pseudo-differential projection which commutes with $D$. Set $Q=\operatorname{Id}-P$ and assume that the restriction $\left.D\right|_{\operatorname{Im} Q}$ of $D$ to the image of $Q$ defines an invertible operator $\left.D\right|_{\operatorname{Im} Q}: I M Q \rightarrow \operatorname{Im} Q$. Then,

$$
\zeta_{\theta}(0, Q, D)=-\operatorname{rank}(\mathrm{Id}-Q) .
$$

In particular, if $m_{0}$ denotes the dimension of the span of the generalized eigenvectors of $D$ corresponding to the eigenvalue $\lambda=0$ (ie $m_{0}$ is the algebraic multiplicity of the eigenvalue $\lambda=0$ of $D$ ), then

$$
\zeta_{\theta}(0, D)=-m_{0}
$$

Proof If $\varepsilon \neq 0$ is a small enough real number, then $D+\varepsilon$ is an invertible differential operator of even order and $\theta$ is an Agmon angle for $D+\varepsilon$. Hence, $\zeta_{\theta}(0, D+\varepsilon)=0$, cf [31]. Clearly,

$$
\zeta_{\theta}(0, Q, D+\varepsilon)=\zeta_{\theta}(0, D+\varepsilon)-\operatorname{rank}(\operatorname{Id}-Q)=-\operatorname{rank}(\operatorname{Id}-Q)
$$


Since 0 is not an eigenvalue of the restriction of $D$ to the image of $Q$, we have

$$
\lim _{\varepsilon \rightarrow 0} \zeta_{\theta}(0, Q, D+\varepsilon)=\zeta_{\theta}(0, Q, D) .
$$

Combining (6-8) and (6-9), we obtain (6-6).

Definition 6.6 The $\zeta$-regularized determinant of $D$ is defined by the formula

$$
\operatorname{Det}_{\theta}^{\prime}(D):=\exp \left(-\left.\frac{d}{d s}\right|_{s=0} \zeta_{\theta}(s, D)\right) .
$$

Roughly speaking, (6-10) says that the logarithm $\log \operatorname{Det}_{\theta}^{\prime}(D)$ of the determinant of $D$ is equal to $-\zeta_{\theta}^{\prime}(0, D)$. However, the logarithm is a multivalued function. Hence, $\log \operatorname{Det}_{\theta}^{\prime}(D)$ is defined only up to a multiple of $2 \pi i$, while $-\zeta_{\theta}^{\prime}(0, D)$ is a well defined complex number. We denote by $\operatorname{LDet}_{\theta}^{\prime}(D)$ the particular value of the logarithm of the determinant such that

$$
\operatorname{LDet}_{\theta}^{\prime}(D)=-\left.\frac{d}{d s}\right|_{s=0} \zeta_{\theta}(s, D)
$$

Let us emphasize that the equality (6-11) is the definition of the number $\operatorname{LDet}_{\theta}^{\prime}(D)$.

Remark 6.7 The prime in $\operatorname{Det}_{\theta}^{\prime}(D)$ and $\operatorname{LDet}_{\theta}^{\prime}(D)$ indicates that we ignore the zero eigenvalues of $D$ in the definition of the regularized determinant. If the operator $D$ is invertible we usually omit the prime and write $\operatorname{Det}_{\theta}(D)$ and $\operatorname{LDet}_{\theta}(D)$ instead.

We will need the following generalization of Definition 6.6.

Definition 6.8 Suppose $Q$ is a 0 th order pseudo-differential projection commuting with $D$. Then $V:=\operatorname{Im} Q$ is a $D$ invariant subspace of $C^{\infty}(M, E)$. The $\zeta$-regularized determinant of the restriction $\left.D\right|_{V}$ of $D$ to $V$ is defined by the formula

$$
\operatorname{Det}_{\theta}^{\prime}\left(\left.D\right|_{V}\right):=e^{\operatorname{LDet}_{\theta}^{\prime}\left(\left.D\right|_{V}\right)},
$$

where

$$
\operatorname{LDet}_{\theta}^{\prime}\left(\left.D\right|_{V}\right)=-\left.\frac{d}{d s}\right|_{s=0} \zeta_{\theta}(s, Q, D)
$$

As in Remark 6.7, if the restriction of $D$ to $V$ defines an invertible operator $\left.D\right|_{V}: V \rightarrow$ $V$, we usually omit the prime in the notation for the numbers (6-12) and (6-13) and write $\operatorname{Det}_{\theta}\left(\left.D\right|_{V}\right)$ and $\operatorname{LDet}_{\theta}\left(\left.D\right|_{V}\right)$ instead.

Remark 6.9 The right hand side of (6-13) is independent of $Q$ except through $\operatorname{Im}(Q)$. This justifies the notation $\operatorname{LDet}_{\theta}^{\prime}\left(\left.D\right|_{V}\right)$. However, we need to know that $V$ is the image of a 0 th order pseudo-differential projection $Q$ to ensure that $\zeta_{\theta}(s, D)$ has a meromorphic extension to the whole $s$-plane with $s=0$ being a regular point. 


\subsection{Spectral subspaces}

We will often use Definition 6.8 in the following special situation. For $\lambda \geq 0$ let $\Pi_{D,[0, \lambda]}$ denote the spectral projection of $D$ corresponding to the set $\{z \in \mathbb{C}:|z| \leq \lambda\}$. It is given by the Cauchy integral

$$
\Pi_{D,[0, \lambda]}=\frac{i}{2 \pi} \int_{0}^{2 \pi}\left(D-(\lambda+\varepsilon) e^{i \phi}\right)^{-1} d \phi,
$$

where $\varepsilon>0$ is small enough so that there are no eigenvalues of $D$ with absolute value in the interval $(\lambda, \lambda+\varepsilon]$. Since the operator $D$ is an elliptic differential operator of order $>0$, the image of $\Pi_{D,[0, \lambda]}$ is finite dimensional and consists of smooth sections. We denote by $C_{[0, \lambda]}^{\infty}(M, E) \subset C^{\infty}(M, E)$ the image of $\Pi_{D,[0, \lambda]}$. Note that $C_{[0, \lambda]}^{\infty}(M, E)$ is equal to the span of the generalized eigenvectors of $D$ corresponding to eigenvalues with absolute value $\leq \lambda$.

Define the projections

$$
\begin{aligned}
\Pi_{D,(\lambda, \infty)} & =\mathrm{Id}-\Pi_{D,[0, \lambda]}, \\
\Pi_{D,(\lambda, \mu]} & =\Pi_{D,[0, \mu]}-\Pi_{D,[0, \lambda]}, \text { for } \mu \geq \lambda .
\end{aligned}
$$

The range of $\Pi_{D,(\lambda, \mu]}$ is finite dimensional and contained in $C^{\infty}(M, E)$. It is equal to the span of the generalized eigenvectors of $D$ with eigenvalues $\chi$ such that $\lambda<|\chi| \leq \mu$. The range of $\Pi_{D,(\lambda, \infty)}$ is infinite dimensional and contains the span of the generalized eigenvectors of $D$ with eigenvalues whose absolute value is greater than $\lambda$, cf Ponge [27, Appendix B].

Let now $\mathcal{I}$ be an interval of the form $[0, \lambda],(\lambda, \mu]$, or $(\lambda, \infty)$. Then $\Pi_{D, \mathcal{I}}$ maps smooth sections to smooth sections and the space

$$
C_{\mathcal{I}}^{\infty}(M, E):=\Pi_{D, \mathcal{I}}\left(C^{\infty}(M, E)\right) \subset C^{\infty}(M, E)
$$

is $D$ invariant. Let $D_{\mathcal{I}}$ denote the restriction of $D$ to the space $C_{\mathcal{I}}^{\infty}(M, E)$. Note also that $D_{\mathcal{I}}$ is invertible whenever $0 \notin \mathcal{I}$.

Definition 6.8 gives us the determinant $\operatorname{Det}_{\theta}^{\prime}\left(D_{\mathcal{I}}\right)$. Clearly, for any $0 \leq \lambda \leq \mu$,

$$
\operatorname{Det}_{\theta}\left(D_{(\lambda, \infty)}\right)=\operatorname{Det}_{\theta}\left(D_{(\lambda, \mu]}\right) \cdot \operatorname{Det}_{\theta}\left(D_{(\mu, \infty)}\right) .
$$

\subsection{Dependence of the determinant on the angle}

Assume that $\theta$ is a principal angle for $D$. Then, $\operatorname{cf}[31 ; 32]$, for any $\varepsilon>0$, we can choose an Agmon angle $\theta^{\prime} \in(\theta-\varepsilon, \theta+\varepsilon)$ for $D$. Let $\theta^{\prime \prime}>\theta^{\prime}$ be another Agmon 
angle for $D$ such that all the angles in the interval $\left[\theta^{\prime}, \theta^{\prime \prime}\right]$ are principal for $D$. Then cf for example, [9, Section 3.10],

$$
\left.\left.\frac{d}{d s}\right|_{s=0} \zeta_{\theta^{\prime}}(s, D) \equiv \frac{d}{d s}\right|_{s=0} \zeta_{\theta^{\prime \prime}}(s, D) \bmod 2 \pi i
$$

Hence, by Definition 6.6,

$$
\operatorname{Det}_{\theta^{\prime \prime}}^{\prime}(D)=\operatorname{Det}_{\theta^{\prime}}^{\prime}(D)
$$

Note that the equality (6-17) holds because both angles, $\theta^{\prime}$ and $\theta^{\prime \prime}$, are close to a given principal angle $\theta$ so that the intersection $\operatorname{spec}(D) \cap L_{\left[\theta^{\prime}, \theta^{\prime \prime}\right]}$ is finite. If there are infinitely many eigenvalues of $D$ in the solid angle $L_{\left[\theta^{\prime}, \theta^{\prime \prime}\right]}$ then $\operatorname{Det}_{\theta^{\prime}}^{\prime}(D)$ and $\operatorname{Det}_{\theta^{\prime \prime}}^{\prime}(D)$ might be different.

\subsection{Graded determinant}

Let $D: C^{\infty}(M, E) \rightarrow C^{\infty}(M, E)$ be a differential operator. Suppose that

$$
Q_{j}: C^{\infty} \rightarrow C^{\infty}(M, E)
$$

with $(j=0, \ldots, d)$ are 0 th order pseudo-differential projections commuting with $D$. Set $V_{j}:=\operatorname{Im} Q_{j}$ and assume that $C^{\infty}(M, E)=\bigoplus_{j=0}^{d} V_{j}$.

Definition 6.13 Assume $\theta \in[0,2 \pi)$ is an Agmon angle for the operator $\left.(-1)^{j} D\right|_{V_{j}}$, for every $j=0, \ldots, d$. The graded determinant $\operatorname{Det}_{\mathrm{gr}, \theta}^{\prime}(D)$ of $D$ (with respect to the grading defined by the pseudo-differential projections $Q_{j}$ ) is defined by the formula

$$
\operatorname{Det}_{\mathrm{gr}, \theta}^{\prime}(D):=e^{\operatorname{LDet}_{\mathrm{gr}, \theta}^{\prime}(D)}
$$

where

$$
\operatorname{LDet}_{\mathrm{gr}, \theta}^{\prime}(D):=\sum_{j=0}^{d}(-1)^{j} \operatorname{LDet}_{\theta}^{\prime}\left(\left.(-1)^{j} D\right|_{V_{j}}\right) .
$$

The following is an important example of the above situation. Let $E=\bigoplus_{j=0}^{d} E_{j}$ be a graded vector bundle over $M$. Suppose that for each $j=0, \ldots, d$, there is a bijective elliptic differential operator

$$
D_{j}: C^{\infty}\left(M, E_{j}\right) \longrightarrow C^{\infty}\left(M, E_{j}\right),
$$

such that $\theta \in[0,2 \pi)$ is an Agmon angle for $(-1)^{j} D_{j}$ for all $j=0, \ldots, d$. We denote by

$$
D=\bigoplus_{j=0}^{d} D_{j}: C^{\infty}(M, E) \longrightarrow C^{\infty}(M, E)
$$


the direct sum of the operators $D_{j}$. Then (6-19) reduces to

$$
\operatorname{LDet}_{\mathrm{gr}, \theta}^{\prime}(D)=\sum_{j=0}^{d}(-1)^{j} \operatorname{LDet}_{\theta}^{\prime}\left((-1)^{j} D_{j}\right) .
$$

\subsection{Case of a self-adjoint leading symbol}

Let $h^{E}$ be a Hermitian metric on the bundle $E \rightarrow M$ and assume that the principal symbol $\sigma(D)(x, \xi)$ of the elliptic operator $D$ is self-adjoint, ie,

$$
\sigma(D)^{*}(x, \xi)=\sigma(D)(x, \xi), \quad(x, \xi) \in T^{*} M,
$$

where $\sigma(D)^{*}(x, \xi)$ denotes the adjoint of the operator $\sigma(D)(x, \xi)$ with respect to the scalar product $h^{E}$. This assumption implies that $D$ can be written as a sum $D=D^{\prime}+A$ where $D^{\prime}$ is a self-adjoint differential operator of order $m$ and $A$ is a differential operator of order smaller than $m$. If the leading symbol of $D$ is self-adjoint then any angle $\theta \neq 0, \pi$ is principal for $D$.

Though the operator $D$ is not self-adjoint in general, the assumption (6-22) guarantees that it has nice spectral properties, cf Markus [24, Section I.6] and [9, Section 3.9]. Though many of the results of this paper remain valid for arbitrary elliptic differential operators which possess an Agmon angle, for simplicity of notation we will often assume that our operators have a self-adjoint leading symbol.

\section{$6.15 \eta$-invariant}

It is well known, cf Singer-Dirac [33] or Wojciechowski [41], that the phase of the determinant of a self-adjoint elliptic differential operator $D$ can be expressed in terms of the $\eta$-invariant of $D$ and the $\zeta$-function of $D^{2}$. We now extend this result to non self-adjoint operators.

First, we recall the definition of the $\eta$-function of $D$ for a non-self-adjoint operator, cf Gilkey [19].

Definition 6.16 Let $D: C^{\infty}(M, E) \rightarrow C^{\infty}(M, E)$ be an elliptic differential operator of order $m \geq 1$ with self-adjoint leading symbol. Assume that $\theta$ is an Agmon angle for $D$ (cf Definition 6.3). Let $\Pi_{>}$(resp. $\Pi_{<}$) be a pseudo-differential projection whose image contains the span of all generalized eigenvectors of $D$ corresponding to eigenvalues $\lambda$ with $\operatorname{Re} \lambda>0$ (resp. with $\operatorname{Re} \lambda<0$ ) and whose kernel contains the span of all generalized eigenvectors of $D$ corresponding to eigenvalues $\lambda$ with $\operatorname{Re} \lambda \leq 0$ (resp. with $\operatorname{Re} \lambda \geq 0$ ), cf [27, Appendix B]. We define the $\eta$-function of $D$ by the formula

$$
\eta_{\theta}(s, D)=\zeta_{\theta}\left(s, \Pi_{>}, D\right)-\zeta_{\theta}\left(s, \Pi_{<,-D)}\right.
$$


Note that, by definition, the purely imaginary eigenvalues of $D$ do not contribute to $\eta_{\theta}(s, D)$.

It was shown by Gilkey, [19], that $\eta_{\theta}(s, D)$ has a meromorphic extension to the whole complex plane $\mathbb{C}$ with isolated simple poles, and that it is regular at 0 . Moreover, the number $\eta_{\theta}(0, D)$ is independent of the Agmon angle $\theta$.

Since the leading symbol of $D$ is self-adjoint, the angles $\pm \pi / 2$ are principal angles for $D$, cf Definition 6.2. In particular, there are at most finitely many eigenvalues of $D$ on the imaginary axis.

Let $m_{+}(D)$ (resp., $m_{-}(D)$ ) denote the number of eigenvalues of $D$, counted with their algebraic multiplicities, on the positive (resp., negative) part of the imaginary axis. Let $m_{0}(D)$ denote algebraic multiplicity of 0 as an eigenvalue of $D$.

Definition 6.17 The $\eta$-invariant $\eta(D)$ of $D$ is defined by the formula

$$
\eta(D)=\frac{1}{2}\left(\eta_{\theta}(0, D)+m_{+}(D)-m_{-}(D)+m_{0}(D)\right) .
$$

As $\eta_{\theta}(0, D)$ is independent of the choice of the Agmon angle $\theta$ for $D$, cf [19], so is $\eta(D)$.

Let $D(t)$ be a smooth 1-parameter family of elliptic operators with self-adjoint leading symbol. Then $\eta(D(t))$ is, in general, not smooth but may have integer jumps when eigenvalues cross the imaginary axis or cross 0 along the imaginary axis. Because of this, the $\eta$-invariant is usually considered modulo integers. However, in this paper we will be interested in the number $e^{i \pi \eta(D)}$, which changes its sign when $\eta(D)$ is changed by an odd integer. Hence, we will consider the $\eta$-invariant as a complex number.

Remark 6.18 Note that our definition of $\eta(D)$ is slightly different from the one proposed by Gilkey in [19]. In fact, in our notation, Gilkey's $\eta$-invariant is given by $\eta(D)+m_{-}(D)$. Hence, reduced modulo integers, the two definitions coincide. However, the number $e^{i \pi \eta(D)}$ will be multiplied by $(-1)^{m_{-}(D)}$ if we replace one definition by the other. In this sense, Definition 6.17 can be viewed as a sign refinement of the definition given in [19].

\subsection{Relationship between the $\eta$-invariant and the determinant}

Since the leading symbol of $D$ is self-adjoint, the angles $\pm \pi / 2$ are principal for $D$. Hence, there exists an Agmon angle $\theta \in(-\pi / 2,0)$ such that there are no eigenvalues of $D$ in the solid angles $L_{(-\pi / 2, \theta]}$ and $L_{(\pi / 2, \theta+\pi]}$. Then $2 \theta$ is an Agmon angle for the operator $D^{2}$. 
Theorem 6.20 Let $D: C^{\infty}(M, E) \rightarrow C^{\infty}(M, E)$ be an elliptic differential operator of order $m \geq 1$ with self-adjoint leading symbol. Assume $\theta \in(-\pi / 2,0)$ is an Agmon angle for $D$ such that there are no eigenvalues of $D$ in the solid angles $L_{(-\pi / 2, \theta]}$ and $L_{(\pi / 2, \theta+\pi]}$ (Hence, there are no eigenvalues of $D^{2}$ in the solid angle $L_{(-\pi, 2 \theta]}$ ). Then $^{2}$

$$
\operatorname{LDet}_{\theta}^{\prime}(D)=\frac{1}{2} \operatorname{LDet}_{2 \theta}^{\prime}\left(D^{2}\right)-i \pi\left(\eta(D)-\frac{\zeta_{2 \theta}\left(0, D^{2}\right)+m_{0}(D)}{2}\right) .
$$

In particular,

$$
\operatorname{Det}_{\theta}^{\prime}(D)=e^{-\frac{1}{2} \zeta_{2 \theta}^{\prime}\left(0, D^{2}\right)} \cdot e^{-i \pi\left(\eta(D)-\frac{\xi_{2 \theta}\left(0, D^{2}\right)+m_{0}(D)}{2}\right)} .
$$

In the case when $D$ is invertible the theorem is proven in [9, Section 4]. The same arguments without any changes prove Theorem 6.20 in the general case.

\section{Remark 6.21}

(a) Let $\theta$ be as in Theorem 6.20 and suppose that $\theta^{\prime} \in(-\pi, 0)$ is another angle such that both $\theta^{\prime}$ and $\theta^{\prime}+\pi$ are Agmon angles for $D$. Then, by (6-17),

$$
\begin{aligned}
\operatorname{Det}_{\theta^{\prime}}^{\prime}(D) & =\operatorname{Det}_{\theta}^{\prime}(D), \\
\zeta_{2 \theta}^{\prime}\left(0, D^{2}\right) & \equiv \zeta_{2 \theta^{\prime}}^{\prime}\left(0, D^{2}\right) \quad \bmod 2 \pi i .
\end{aligned}
$$

In particular,

$$
e^{-\frac{1}{2} \zeta_{2 \theta^{\prime}}^{\prime}\left(0, D^{2}\right)}= \pm e^{-\frac{1}{2} \zeta_{2 \theta}^{\prime}\left(0, D^{2}\right)} .
$$

Clearly, $\zeta_{\theta_{1}}\left(0, D^{2}\right)=\zeta_{\theta_{2}}\left(0, D^{2}\right)$ if there are finitely many eigenvalues of $D^{2}$ in the solid angle $L_{\left[\theta_{1}, \theta_{2}\right]}$. Hence, $\zeta_{2 \theta}\left(0, D^{2}\right)=\zeta_{2 \theta^{\prime}}\left(0, D^{2}\right)$. We then conclude from (6-26), (6-27), and (6-28) that

$$
\operatorname{Det}_{\theta^{\prime}}^{\prime}(D)= \pm e^{-\frac{1}{2} \zeta_{2 \theta^{\prime}}^{\prime}\left(0, D^{2}\right)} \cdot e^{-i \pi\left(\eta(D)-\frac{\xi_{2 \theta^{\prime}}\left(0, D^{2}\right)+m_{0}(D)}{2}\right)} .
$$

In other words, for (6-26) to hold we need the precise assumption on $\theta$ which are specified in Theorem 6.20. But "up to a sign" it holds for every spectral cut in the lower half plane.

(b) If instead of the spectral cut $R_{\theta}$ in the lower half-plane we use the spectral cut $R_{\theta+\pi}$ in the upper half-plane we will get a similar formula

$$
\operatorname{LDet}_{\theta+\pi}^{\prime}(D)=\frac{1}{2} \operatorname{LDet}_{2 \theta}^{\prime}\left(D^{2}\right)+i \pi\left(\eta(D)-\frac{\zeta_{2 \theta}\left(0, D^{2}\right)+m_{0}(D)}{2}\right),
$$

\footnotetext{
${ }^{2}$ Recall that we denote by $\operatorname{LDet}_{\theta}^{\prime}(D)$ the particular branch of the logarithm of the determinant of $D$ defined by formula (6-11).
} 
whose proof is a verbatim repetition of the proof of (6-25), cf [9, Section 4].

(c) If the dimension of $M$ is odd, then, in view of Proposition $6.5, \zeta_{2 \theta}\left(0, D^{2}\right)=$ $-m_{0}(D)$. Hence, (6-25) simplifies to

$$
\operatorname{LDet}_{\theta}^{\prime}(D)=\frac{1}{2} \operatorname{LDet}_{2 \theta}^{\prime}\left(D^{2}\right)-i \pi \eta(D) .
$$

\subsection{2 $\eta$-invariant and graded determinant}

Suppose now that $D=\bigoplus_{j=0}^{d} D_{j}$ is as in (6-20). Choose $\theta \in(-\pi / 2,0)$ such that there are no eigenvalues of $D_{j}$ in the solid angles $L_{(-\pi / 2, \theta]}$ and $L_{(\pi / 2, \theta+\pi]}$ for every $0 \leq j \leq d$. From Definition 6.17 of the $\eta$-invariant it follows that

$$
\eta\left(-D_{j}\right)=-\eta\left(D_{j}\right)+m_{0}\left(D_{j}\right) .
$$

Combining this latter equality with (6-21) and (6-25) we obtain

$$
\begin{aligned}
\operatorname{LDet}_{\mathrm{gr}, \theta}^{\prime}(D)=\frac{1}{2} \sum_{j=0}^{d}(-1)^{j} & \operatorname{LDet}_{2 \theta}^{\prime}\left(D_{j}^{2}\right) \\
& \quad-i \pi\left(\eta(D)-\frac{m_{0}(D)}{2}-\frac{1}{2} \sum_{j=0}^{d}(-1)^{j} \zeta_{2 \theta}\left(0, D_{j}^{2}\right)\right),
\end{aligned}
$$

where $\eta(D)=\sum_{j=0}^{d} \eta\left(D_{j}\right)$ is the $\eta$-invariant of the operator $D=\bigoplus_{j=0}^{d} D_{j}$ and $m_{0}(D)=\sum_{j=0}^{d} m_{0}\left(D_{j}\right)$ is the algebraic multiplicity of 0 as an eigenvalue of $D$.

Finally, note that, by Remark 6.21(c), if the dimension of $M$ is odd, and all the operators $D_{j}$ are invertible (so that $m_{0}\left(D_{j}\right)=0$ ), then (6-32) takes the form

$$
\operatorname{LDet}_{\mathrm{gr}, \theta}(D)=\frac{1}{2} \sum_{j=0}^{d}(-1)^{j} \operatorname{LDet}_{2 \theta}\left(D_{j}^{2}\right)-i \pi \eta(D) .
$$

\subsection{Generalization}

The definition (6-24) of the $\eta$-invariant easily generalizes to operators acting on a subspace of the space $C^{\infty}(M, E)$ of smooth sections of the vector bundle $E$, cf [9, Section 4.10].

Let $D: C^{\infty}(M, E) \rightarrow C^{\infty}(M, E)$ be an elliptic differential operator with a self-adjoint leading symbol. Let $Q: C^{\infty}(M, E) \rightarrow C^{\infty}(M, E)$ be a 0-th order pseudo-differential projection commuting with $D$. Then $V:=\operatorname{Im} Q \subset C^{\infty}(M, E)$ is a $D$-invariant 
subspace. Let $\Pi_{>}$and $\Pi_{<}$be as in Definition 6.16. Let $\theta$ be as in Section 6.22 and set

$$
\begin{gathered}
\eta_{\theta}\left(s,\left.D\right|_{V}\right)=\zeta_{\theta}\left(s, Q \Pi_{>}, D\right)-\zeta_{\theta}\left(s, Q \Pi_{<,-D),}\right. \\
\eta\left(\left.D\right|_{V}\right)=\frac{1}{2}\left(\eta_{\theta}\left(0,\left.D\right|_{V}\right)+m_{+}\left(\left.D\right|_{V}\right)-m_{-}\left(\left.D\right|_{V}\right)+m_{0}\left(\left.D\right|_{V}\right)\right) .
\end{gathered}
$$

Then, cf [9, Section 4.10],

$$
\begin{aligned}
& \operatorname{LDet}_{\theta}^{\prime}\left(\left.D\right|_{V}\right) \\
& \qquad=\frac{1}{2} \operatorname{LDet}_{2 \theta}^{\prime}\left(\left.D^{2}\right|_{V}\right)-i \pi\left(\eta\left(\left.D\right|_{V}\right)-\frac{\zeta_{2 \theta}\left(0,\left.D^{2}\right|_{V}\right)+m_{0}\left(\left.D\right|_{V}\right)}{2}\right),
\end{aligned}
$$

where we used the notation

$$
\zeta_{2 \theta}\left(s,\left.D^{2}\right|_{V}\right)=\zeta_{2 \theta}\left(s, Q, D^{2}\right),
$$

$\mathrm{cf}(6-5)$.

Note, however, that an analogue of (6-31) does not necessarily hold in this case even if $\operatorname{dim} M$ is odd, because $\zeta_{2 \theta}\left(s,\left.D^{2}\right|_{V_{j}}\right)$ defined by (6-37), is not a $\zeta$-function of a differential operator and Proposition 6.5 does not necessarily hold.

Finally, suppose that $V=\bigoplus_{j=0}^{d} V_{j}$ is given as in Definition 6.13. Then

$$
\begin{aligned}
\operatorname{LDet}_{\mathrm{gr}, \theta}^{\prime}(D)= & \frac{1}{2} \sum_{j=0}^{d}(-1)^{j} \operatorname{LDet}_{2 \theta}^{\prime}\left(\left.D^{2}\right|_{V_{j}}\right) \\
& -i \pi\left(\eta\left(\left.D\right|_{V}\right)-\frac{m_{0}\left(\left.D\right|_{V}\right)}{2}-\frac{1}{2} \sum_{j=0}^{d}(-1)^{j} \zeta_{2 \theta}\left(0,\left.D^{2}\right|_{V_{j}}\right)\right),
\end{aligned}
$$

where $\eta\left(\left.D\right|_{V}\right)=\sum_{j=0}^{d} \eta\left(\left.D\right|_{V_{j}}\right)$ and $m_{0}\left(\left.D\right|_{V}\right)=\sum_{j=0}^{d} m_{0}\left(\left.D\right|_{V_{j}}\right)$.

\section{The graded determinant of the odd signature operator}

In this section we define the graded determinant of the Atiyah-Patodi-Singer odd signature operator, [2;19], of a flat vector bundle $E$ over a closed oriented Riemannian manifold $M$. We also use this determinant to define an element $\rho$ of the determinant line of the cohomology of the bundle $E$. Our definition is based on the formula which relates the graded determinant of the signature operator and the refined torsion in the finite dimensional setting, cf Proposition 5.10. In Section 11 we will show that, if $E$ admits an invariant Hermitian metric, then the Ray-Singer norm of $\rho$ is equal to 1 . 
Thus the element $\rho$ can be viewed as a refinement of the Ray-Singer metric. In general, however, it might depend on the Riemannian metric on $M$. In subsequent sections we study the metric anomaly of $\rho$, use it to "correct" $\rho$, and then define a differential invariant of the flat bundle $E$ - a metric independent element of the determinant line of the cohomology, called the refined analytic torsion.

\subsection{Setting}

Let $M$ be a smooth closed oriented manifold of odd dimension $d=2 r-1$ and let $E \rightarrow M$ be a complex vector bundle over $M$ endowed with a flat connection $\nabla$. We denote by $\nabla$ also the induced differential

$$
\nabla: \Omega^{\bullet}(M, E) \longrightarrow \Omega^{\bullet+1}(M, E),
$$

where $\Omega^{k}(M, E)$ denotes the space of smooth differential forms on $M$ of degree $k$ with values in $E$.

\subsection{Odd signature operator}

Fix a Riemannian metric $g^{M}$ on $M$ and let $*: \Omega^{\bullet}(M, E) \rightarrow \Omega^{d-\bullet}(M, E)$ denote the Hodge $*$-operator. Define the chirality operator $\Gamma=\Gamma\left(g^{M}\right): \Omega^{\bullet}(M, E) \rightarrow$ $\Omega^{\bullet}(M, E)$ by the formula

$$
\Gamma \omega:=i^{r}(-1)^{\frac{k(k+1)}{2}} * \omega, \quad \omega \in \Omega^{k}(M, E),
$$

with $r$ given as above by $r=\frac{1}{2}(d+1)$. This operator is equal to the operator defined in [3, Section 3.2] as one can see by applying [3, Proposition 3.58] in the case $\operatorname{dim} M$ is odd. In particular, $\Gamma^{2}=1$.

Definition 7.3 The odd signature operator is the operator

$$
\mathcal{B}=\mathcal{B}\left(\nabla, g^{M}\right):=\Gamma \nabla+\nabla \Gamma: \Omega^{\bullet}(M, E) \longrightarrow \Omega^{\bullet}(M, E) .
$$

We denote by $\mathcal{B}_{k}$ the restriction of $\mathcal{B}$ to the space $\Omega^{k}(M, E)$.

More explicitly, the value of the odd signature operator on a form $\omega \in \Omega^{k}(M, E)$ is given by the formula

$\mathcal{B}_{k} \omega:=i^{r}(-1)^{\frac{k(k+1)}{2}+1}\left((-1)^{k} * \nabla-\nabla *\right) \omega \in \Omega^{d-k-1}(M, E) \oplus \Omega^{d-k+1}(M, E)$.

The odd signature operator was introduced by Atiyah, Patodi, and Singer, [1, page 44], [2, page 405], in the case when $E$ is endowed with a Hermitian metric invariant with respect to $\nabla$ (ie invariant under parallel transport by $\nabla$ ). The general case was studied by Gilkey, [19, pages 64-65]. 
Lemma 7.4 Suppose that $E$ is endowed with a Hermitian metric $h^{E}$. Denote by $\langle\cdot, \cdot\rangle$ the scalar product on $\Omega^{\bullet}(M, E)$ induced by $h^{E}$ and the Riemannian metric $g^{M}$ on $M$. Then

(1) $\mathcal{B}$ is elliptic and its leading symbol is symmetric with respect to the Hermitian metric $h^{E}$.

(2) If, in addition, the metric $h^{E}$ is invariant with respect to the connection $\nabla$, then $\mathcal{B}$ is symmetric with respect to the scalar product $\langle\cdot, \cdot\rangle$,

$$
\mathcal{B}^{*}=\mathcal{B} \text {. }
$$

If the metric $h^{E}$ is not invariant, then, in general, $\mathcal{B}$ is not symmetric.

The proof of the lemma is a simple calculation. The first part is already stated in [2, page 405]. The second part is proven in the Remark on page 65 of [19].

\subsection{Decomposition of the odd signature operator}

Set

$$
\begin{aligned}
& \Omega^{\text {even }}(M, E):=\bigoplus_{p=0}^{r-1} \Omega^{2 p}(M, E), \quad \Omega^{\text {odd }}(M, E):=\bigoplus_{p=1}^{r} \Omega^{2 p-1}(M, E), \\
& \mathcal{B}_{\text {even }}:=\bigoplus_{p=0}^{r-1} \mathcal{B}_{2 p}: \Omega^{\text {even }}(M, E) \longrightarrow \Omega^{\text {even }}(M, E), \\
& \mathcal{B}_{\text {odd }}:=\bigoplus_{p=1}^{r} \mathcal{B}_{2 p-1}: \Omega^{\text {odd }}(M, E) \longrightarrow \Omega^{\text {odd }}(M, E) .
\end{aligned}
$$

Since $\Gamma^{2}=1$ we obtain

$$
\mathcal{B}_{\text {odd }}=\left.\Gamma \circ \mathcal{B}_{\text {even }} \circ \Gamma\right|_{\Omega^{\text {odd }}(M, E)} .
$$

Hence, the whole information about the odd signature operator is encoded in its even part $\mathcal{B}_{\text {even }}$. The operator $\mathcal{B}_{\text {even }}$ can be expressed by the following formula, which is slightly simpler than (7-3).

$$
\mathcal{B}_{\text {even }} \omega:=i^{r}(-1)^{p+1}(* \nabla-\nabla *) \omega, \text { for } \omega \in \Omega^{2 p}(M, E) .
$$

Note that for each $k=0, \ldots, d$, the operator $\mathcal{B}^{2}$ maps $\Omega^{k}(M, E)$ into itself. Suppose $\mathcal{I}$ is an interval of the form $[0, \lambda],(\lambda, \mu]$, or $(\lambda, \infty](\mu \geq \lambda \geq 0)$. Then $\Pi_{\mathcal{B}^{2}, \mathcal{I}}$ is the spectral projection of $\mathcal{B}^{2}$ corresponding to $\mathcal{I}$, cf Section 6.10. Set

$$
\Omega_{\mathcal{I}}^{\bullet}(M, E):=\Pi_{\mathcal{B}^{2}, \mathcal{I}}\left(\Omega^{\bullet}(M, E)\right) \subset \Omega^{\bullet}(M, E) .
$$


Recall from Section 6.10, that if the interval $\mathcal{I}$ is bounded, then the space $\Omega_{\mathcal{I}}^{\bullet}(M, E)$ is finite dimensional and is equal to the span of the generalized eigenforms of $\mathcal{B}^{2}$ corresponding to eigenvalues with absolute value $\leq \lambda$. In general, $\Omega_{\mathcal{I}}^{\bullet}(M, E)$ contains the span of the eigenforms of $\mathcal{B}^{2}$ corresponding to eigenvalues whose absolute value lies in $\mathcal{I}$.

For each $k=0, \ldots, d$, set

$$
\begin{aligned}
& \Omega_{+, \mathcal{I}}^{k}(M, E):=\operatorname{Ker}(\nabla \Gamma) \cap \Omega_{\mathcal{I}}^{k}(M, E)=(\Gamma(\operatorname{Ker} \nabla)) \cap \Omega_{\mathcal{I}}^{k}(M, E) ; \\
& \Omega_{-, \mathcal{I}}^{k}(M, E):=\operatorname{Ker}(\Gamma \nabla) \cap \Omega_{\mathcal{I}}^{k}(M, E)=\operatorname{Ker} \nabla \cap \Omega_{\mathcal{I}}^{k}(M, E) .
\end{aligned}
$$

Clearly,

$$
\Omega_{\mathcal{I}}^{k}(M, E)=\Omega_{+, \mathcal{I}}^{k}(M, E) \oplus \Omega_{-, \mathcal{I}}^{k}(M, E) \text {, if } 0 \notin \mathcal{I} .
$$

We consider the decomposition (7-7) as a grading ${ }^{3}$ of the space $\Omega_{\mathcal{I}}^{\bullet}(M, E)$, and refer to $\Omega_{+, \mathcal{I}}^{k}(M, E)$ and $\Omega_{-, \mathcal{I}}^{k}(M, E)$ as the positive and negative subspaces of $\Omega_{\mathcal{I}}^{k}(M, E)$.

As both, $\Gamma$ and $\nabla$, commute with $\mathcal{B}^{2}$, we conclude that, for $k=0, \ldots, d$,

$$
\Gamma: \Omega_{ \pm, \mathcal{I}}^{k}(M, E) \longrightarrow \Omega_{\mp, \mathcal{I}}^{d-k}(M, E),
$$

and

$$
\nabla: \Omega_{ \pm, \mathcal{I}}^{k}(M, E) \longrightarrow \Omega_{\mp, \mathcal{I}}^{k+1}(M, E) .
$$

Denote by $\mathcal{B}_{k}^{\mathcal{I}}$ the restriction of $\mathcal{B}$ to $\Omega_{\mathcal{I}}^{k}(M, E)$ and by $\mathcal{B}_{k}^{ \pm, \mathcal{I}}$ the restriction of $\mathcal{B}$ to $\Omega_{ \pm, \mathcal{I}}^{k}(M, E)$. Then

$$
\begin{aligned}
\mathcal{B}_{k}^{+, \mathcal{I}}: \Omega_{+, \mathcal{I}}^{k}(M, E) \longrightarrow \Omega_{+, \mathcal{I}}^{d-k-1}(M, E), & \omega \mapsto \Gamma \nabla \omega ; \\
\mathcal{B}_{k}^{-, \mathcal{I}}: \Omega_{-, \mathcal{I}}^{k}(M, E) \longrightarrow \Omega_{-, \mathcal{I}}^{d-k+1}(M, E), & \omega \mapsto \nabla \Gamma \omega .
\end{aligned}
$$

\subsection{Graded determinant of the odd signature operator}

Let $\mathcal{I}$ be an interval of the form $[0, \lambda],(\lambda, \mu]$, or $(\lambda, \infty](\mu \geq \lambda \geq 0)$ and define

$$
\Omega_{ \pm, \mathcal{I}}^{\mathrm{even}}(M, E)=\bigoplus_{p=0}^{r-1} \Omega_{ \pm, \mathcal{I}}^{2 p}(M, E) .
$$

\footnotetext{
${ }^{3}$ Note, that our grading is opposite to the one considered in [11, Section 2].
} 
Let $\mathcal{B}^{\mathcal{I}}, \mathcal{B}_{\text {even }}^{\mathcal{I}}$, and $\mathcal{B}_{\text {odd }}^{\mathcal{I}}$ denote the restrictions of $\mathcal{B}$ to the subspaces $\Omega_{\mathcal{I}}^{\bullet}(M, E)$, $\Omega_{\mathcal{I}}^{\text {even }}(M, E)$, and $\Omega_{\mathcal{I}}^{\text {odd }}(M, E)$ respectively. Then

$$
\mathcal{B}_{\text {even }}^{\mathcal{I}}: \Omega_{ \pm, \mathcal{I}}^{\text {even }}(M, E) \longrightarrow \Omega_{ \pm, \mathcal{I}}^{\text {even }}(M, E) .
$$

Let $\mathcal{B}_{\text {even }}^{ \pm, \mathcal{I}}$ denote the restriction of $\mathcal{B}_{\text {even }}^{\mathcal{I}}$ to the space $\Omega_{ \pm, \mathcal{I}}^{\text {even }}(M, E)$. Clearly, the operators $\mathcal{B}_{\text {even }}^{ \pm, \mathcal{I}}$ are bijective whenever $0 \notin \mathcal{I}$.

By Definition 5.5 and Definition 6.13, for every $\mathcal{I}$, the graded determinant of $\mathcal{B}_{\text {even }}^{\mathcal{I}}$ is given by the formula

$$
\operatorname{Det}_{\mathrm{gr}, \theta}^{\prime}\left(\mathcal{B}_{\mathrm{even}}^{\mathcal{I}}\right):=e^{\mathrm{LDet}_{\mathrm{gr}, \theta}^{\prime}\left(\mathcal{B}_{\mathrm{even}}^{\mathcal{I}}\right)},
$$

where $\theta \in(-\pi, 0)$ is an Agmon angle for the operator $\mathcal{B}_{\text {even }}^{\mathcal{I}}$, and

$$
\operatorname{LDet}_{\mathrm{gr}, \theta}^{\prime}\left(\mathcal{B}_{\text {even }}^{\mathcal{I}}\right):=\operatorname{LDet}_{\theta}^{\prime}\left(\mathcal{B}_{\text {even }}^{+, \mathcal{I}}\right)-\operatorname{LDet}_{\theta}^{\prime}\left(-\mathcal{B}_{\text {even }}^{-, \mathcal{I}}\right) \in \mathbb{C} \text {. }
$$

Clearly, for $0 \leq \lambda \leq \mu$, we have

$$
\operatorname{Det}_{\mathrm{gr}, \theta}\left(\mathcal{B}_{\text {even }}^{(\lambda, \infty)}\right)=\operatorname{Det}_{\mathrm{gr}, \theta}\left(\mathcal{B}_{\text {even }}^{(\lambda, \mu]}\right) \cdot \operatorname{Det}_{\mathrm{gr}, \theta}\left(\mathcal{B}_{\text {even }}^{(\mu, \infty)}\right)
$$

Note also that since the rank of $\mathcal{B}_{\text {even }}^{(\lambda, \mu]}$ is finite, $\operatorname{Det}_{\mathrm{gr}, \theta}\left(\mathcal{B}_{\text {even }}^{(\lambda, \mu]}\right)$ is independent of $\theta$ and is equal to the product of the eigenvalues of $\mathcal{B}_{\text {even }}^{(\lambda, \mu]}$.

\subsection{The canonical element of the determinant line}

Since the covariant differentiation $\nabla$ commutes with $\mathcal{B}$, the subspace $\Omega_{\mathcal{I}}^{\bullet}(M, E)$ is a subcomplex of the twisted de Rham complex $\left(\Omega^{\bullet}(M, E), \nabla\right)$. Clearly, for each $\lambda \geq 0$, the complex $\Omega_{(\lambda, \infty)}^{\bullet}(M, E)$ is acyclic. Since

$$
\Omega^{\bullet}(M, E)=\Omega_{[0, \lambda]}^{\bullet}(M, E) \oplus \Omega_{(\lambda, \infty)}^{\bullet}(M, E),
$$

the cohomology $H_{[0, \lambda]}^{\bullet}(M, E)$ of the complex $\Omega_{[0, \lambda]}^{\bullet}(M, E)$ is naturally isomorphic to the cohomology $H^{\bullet}(M, E)$ of $\Omega^{\bullet}(M, E)$.

Let $\Gamma_{\mathcal{I}}$ denote the restriction of $\Gamma$ to $\Omega_{\mathcal{I}}^{\bullet}(M, E)$. For each $\lambda \geq 0$, let

$$
\rho_{\Gamma_{[0, \lambda]}}=\rho_{\Gamma_{[0, \lambda]}}\left(\nabla, g^{M}\right) \in \operatorname{Det}\left(H_{[0, \lambda]}^{\bullet}(M, E)\right)
$$

denote the refined torsion of the finite dimensional complex $\left(\Omega_{[0, \lambda]}^{\bullet}(M, E), \nabla\right)$ corresponding to the chirality operator $\Gamma_{[0, \lambda]}$, cf Definition 4.3. We view $\rho_{\Gamma_{[0, \lambda]}}$ as an element of $\operatorname{Det}\left(H^{\bullet}(M, E)\right)$ via the canonical isomorphism between $H_{[0, \lambda]}^{\bullet}(M, E)$ and $H^{\bullet}(M, E)$.

From Proposition 5.10, (7-12), and (6-17), we immediately obtain the following 
Proposition 7.8 Assume that $\theta \in(-\pi, 0)$ is an Agmon angle for the operator $\mathcal{B}_{\text {even }}$. Then the element

$$
\rho=\rho\left(\nabla, g^{M}\right):=\operatorname{Det}_{\mathrm{gr}, \theta}\left(\mathcal{B}_{\text {even }}^{(\lambda, \infty)}\right) \cdot \rho_{\Gamma_{[0, \lambda]}} \in \operatorname{Det}\left(H^{\bullet}(M, E)\right)
$$

is independent of the choice of $\lambda \geq 0$. Further, $\rho$ is independent of the choice of the Agmon angle $\theta \in(-\pi, 0)$ of $\mathcal{B}_{\text {even }}$.

If the odd signature operator is invertible then $H^{\bullet}(M, E)=0$. In this case, $\operatorname{Det}\left(H^{\bullet}(M, E)\right)$ is canonically isomorphic to $\mathbb{C}$ and $\rho_{\Gamma_{\{0\}}}=1$. Hence, $\rho$ is a complex number which coincides with the graded determinant $\operatorname{Det}_{\mathrm{gr}, \theta}\left(\mathcal{B}_{\mathrm{even}}\right)=\operatorname{Det}_{\mathrm{gr}, \theta}\left(\mathcal{B}_{\text {even }}^{(0, \infty)}\right)$. This case was studied in [9].

\section{Relationship with the $\eta$-invariant}

In this section, we study the relationship between the graded determinant (7-10) and the $\eta$-invariant of $\mathcal{B}_{\text {even }}^{(\lambda, \infty)}$. For the special case when $\mathcal{B}$ is bijective and $\lambda=0$ this relationship was established in [9, Section 7].

To simplify the notation set

$$
\eta_{\lambda}=\eta_{\lambda}\left(\nabla, g^{M}\right):=\eta\left(\mathcal{B}_{\text {even }}^{(\lambda, \infty)}\right),
$$

and

$$
\begin{aligned}
\xi_{\lambda}=\xi_{\lambda}\left(\nabla, g^{M}, \theta\right) & :=\frac{1}{2} \sum_{j=0}^{d-1}(-1)^{j} \operatorname{LDet}_{2 \theta}\left(\mathcal{B}_{d-j-1}^{+,(\lambda, \infty)} \circ \mathcal{B}_{j}^{+,(\lambda, \infty)}\right) \\
& =\frac{1}{2} \sum_{j=0}^{d-1}(-1)^{j} \operatorname{LDet}_{2 \theta}\left(\left.(\Gamma \nabla)^{2}\right|_{\Omega_{+,(\lambda, \infty)}^{j}(M, E)}\right),
\end{aligned}
$$

where $\theta \in(-\pi / 2,0)$ and both, $\theta$ and $\theta+\pi$, are Agmon angles for $\mathcal{B}_{\text {even }}$ (hence, $2 \theta$ is an Agmon angle for $\mathcal{B}_{\text {even }}^{2}$ ).

It is shown in [9, Section 8.4] that 4

$$
\begin{aligned}
\xi_{\lambda} & :=\frac{1}{2} \sum_{j=0}^{d}(-1)^{j+1} j \operatorname{LDet}_{2 \theta}\left[\left.\left(\mathcal{B}^{(\lambda, \infty)}\right)^{2}\right|_{\Omega_{(\lambda, \infty)}^{j}(M, E)}\right] \\
& =\frac{1}{2} \sum_{j=0}^{d}(-1)^{j+1} j \operatorname{LDet}_{2 \theta}\left[\left.\left((\Gamma \nabla)^{2}+(\nabla \Gamma)^{2}\right)\right|_{\Omega_{(\lambda, \infty)}^{j}(M, E)}\right] .
\end{aligned}
$$

\footnotetext{
${ }^{4}$ In [9] we only considered the case when $\mathcal{B}$ is bijective and $\lambda=0$. But the arguments leading to [9, formula (8.7)] work without any changes in our more general situation.
} 
Set

$$
d_{j, \lambda}:=\operatorname{dim} \Omega_{[0, \lambda]}^{j}(M, E), \quad j=0, \ldots, d .
$$

Proposition 8.1 Let $\nabla$ be a flat connection on a vector bundle $E$ over a closed Riemannian manifold $\left(M, g^{M}\right)$ of odd dimension $d=2 r-1$. Assume $\theta \in(-\pi / 2,0)$ is an Agmon angle for the odd signature operator $\mathcal{B}=\mathcal{B}\left(\nabla, g^{M}\right)$ such that there are no eigenvalues of $\mathcal{B}$ in the solid angles $L_{(-\pi / 2, \theta]}$ and $L_{(\pi / 2, \theta+\pi]}$. Then, for every $\lambda \geq 0$,

$$
\operatorname{LDet}_{\mathrm{gr}, \theta}\left(\mathcal{B}_{\text {even }}^{(\lambda, \infty)}\right)=\xi_{\lambda}-i \pi \eta_{\lambda}-\frac{i \pi}{2} \sum_{j=0}^{d}(-1)^{j} j d_{j, \lambda}
$$

Proof Since the operator $B_{\text {even }}^{(\lambda, \infty)}$ has no zero eigenvalues, we conclude from (6-38), that to prove (8-5) it is enough to show the following two identities

$$
\begin{gathered}
2 \xi_{\lambda}=\operatorname{LDet}_{2 \theta}\left(\mathcal{B}_{\text {even }}^{+,(\lambda, \infty)}\right)^{2}-\operatorname{LDet}_{2 \theta}\left(\mathcal{B}_{\text {even }}^{-,(\lambda, \infty)}\right)^{2} \\
\zeta_{2 \theta}\left(0,\left(\mathcal{B}_{\text {even }}^{+,(\lambda, \infty)}\right)^{2}\right)-\zeta_{2 \theta}\left(0,\left(\mathcal{B}_{\text {even }}^{-,(\lambda, \infty)}\right)^{2}\right)=\sum_{j=0}^{d}(-1)^{j} j d_{j, \lambda} .
\end{gathered}
$$

A verbatim repetition of the arguments which led to [9, formula (7.17)] implies that

$$
\begin{aligned}
\zeta_{2 \theta}\left(s,\left(\mathcal{B}_{\text {even }}^{+,(\lambda, \infty)}\right)^{2}\right)-\zeta_{2 \theta}\left(s,\left(\mathcal{B}_{\text {even }}^{-,(\lambda, \infty)}\right)^{2}\right) & \\
& =\sum_{j=0}^{d}(-1)^{j+1} j \zeta_{2 \theta}\left(s,\left.\left(\mathcal{B}^{(\lambda, \infty)}\right)^{2}\right|_{\Omega^{j}(M, E)}\right) .
\end{aligned}
$$

From (8-3) and (8-8) we obtain

$$
2 \xi_{\lambda}=\left.\frac{d}{d s}\right|_{s=0}\left[\zeta_{2 \theta}\left(s,\left(\mathcal{B}_{\text {even }}^{+}\right)^{2}\right)-\zeta_{2 \theta}\left(s,\left(\mathcal{B}_{\text {even }}^{-}\right)^{2}\right)\right] .
$$

Hence (8-6) is established.

Combining (8-8) and Proposition 6.5 we obtain (8-7).

\section{The metric anomaly of $\rho$ and the definition of the refined analytic torsion}

In this section we study the dependence of the element $\rho=\rho\left(\nabla, g^{M}\right)$ defined in (7-15) on the Riemannian metric $g^{M}$. In particular, we show that, if $\operatorname{dim} M=2 r-1 \equiv$ 
$1(\bmod 4)$, then $\rho$ is independent of $g^{M}$. We then use these results to construct the refined analytic torsion - a canonical element of $\operatorname{Det}\left(H^{\bullet}(M, F)\right)$ which is independent of the metric, ie, is a differential invariant of the flat vector bundle $(E, \nabla)$.

\subsection{Relationship between $\rho(t)$ and the $\eta$-invariant}

Suppose that $g_{t}^{M}, t \in \mathbb{R}$, is a smooth family of Riemannian metrics on $M$. Let

$$
\rho(t)=\rho\left(\nabla, g_{t}^{M}\right) \in \operatorname{Det}\left(H^{\bullet}(M, E)\right)
$$

be the canonical element defined in (7-15).

Let $\Gamma_{t}$ denote the chirality operator corresponding to the metric $g_{t}^{M}, \mathrm{cf}(7-1)$, and let $\mathcal{B}(t)=\mathcal{B}\left(\nabla, g_{t}^{M}\right)$ denote the odd signature operator corresponding to the Riemannian metric $g_{t}^{M}$.

Fix $t_{0} \in \mathbb{R}$ and choose $\lambda \geq 0$ so that there are no eigenvalues of $\mathcal{B}\left(t_{0}\right)^{2}$ whose absolute values are equal to $\lambda$. Then there exists $\delta>0$ such that the same is true for all $t \in\left(t_{0}-\delta, t_{0}+\delta\right)$. In particular, if we denote by $\Omega_{[0, \lambda], t}^{\bullet}(M, E)$ the span of the generalized eigenvectors of $\mathcal{B}(t)^{2}$ corresponding to eigenvalues with absolute value $\leq \lambda$, then $\operatorname{dim} \Omega_{[0, \lambda], t}^{\bullet}(M, E)$ is independent of $t \in\left(t_{0}-\delta, t_{0}+\delta\right)$. We set

$$
d_{j, \lambda}:=\operatorname{dim} \Omega_{[0, \lambda], t}^{j}(M, E), \quad j=0, \ldots, d, \quad t \in\left(t_{0}-\delta, t_{0}+\delta\right) .
$$

By definition (7-15),

$$
\rho(t)=\operatorname{Det}_{\mathrm{gr}, \theta}\left(\mathcal{B}_{\text {even }}^{(\lambda, \infty)}(t)\right) \cdot \rho_{\Gamma_{t,[0, \lambda]}} .
$$

For each $t \in\left(t_{0}-\delta, t_{0}+\delta\right)$ and $\theta \in(-\pi / 2,0)$, such that $\theta$ and $\theta+\pi$ are Agmon angles for $\mathcal{B}^{(\lambda, \infty)}(t)$, let us introduce the following short notation for the quantities introduced in (8-1) and (8-2)

$$
\xi_{\lambda}(t, \theta):=\xi_{\lambda}\left(\nabla, g_{t}^{M}, \theta\right), \quad \eta_{\lambda}(t):=\eta_{\lambda}\left(\nabla, g_{t}^{M}\right) .
$$

Assume that $\theta_{0} \in(-\pi / 2,0)$ is an Agmon angle for $\mathcal{B}\left(t_{0}\right)=\mathcal{B}\left(\nabla, g_{t_{0}}^{M}\right)$ such that there are no eigenvalues of $\mathcal{B}\left(t_{0}\right)$ in the solid angles $L_{\left(-\pi / 2, \theta_{0}\right]}$ and $L_{\left(\pi / 2, \theta_{0}+\pi\right)}$. Choose $\delta$, if necessary, smaller, so that for every $t \in\left(t_{0}-\delta, t_{0}+\delta\right)$ and every $j=0, \ldots, d$ both, $\theta_{0}$ and $\theta_{0}+\pi$, are Agmon angles of $\mathcal{B}_{j}^{(\lambda, \infty)}(t)$. For $t \neq t_{0}$ it might happen that there are eigenvalues of $\mathcal{B}^{(\lambda, \infty)}(t)$ in $L_{\left(-\pi / 2, \theta_{0}\right)}$ and/or $L_{\left(\pi / 2, \theta_{0}+\pi\right)}$. Hence, (8-5) is not necessarily true, in general, for $t \neq t_{0}$. However, from (6-16) and (8-2), we conclude that for every angle $\theta \in(-\pi / 2,0)$, so that $\theta$ and $\theta+\pi$ are Agmon angles for $\mathcal{B}^{(\lambda, \infty)}(t)$ (and, hence, $2 \theta$ is an Agmon angle for $\mathcal{B}^{(\lambda, \infty)}(t)^{2}$ ),

$$
\xi_{\lambda}(t, \theta) \equiv \xi_{\lambda}\left(t, \theta_{0}\right) \quad \bmod \pi i
$$


Thus, from (8-5), we obtain

$$
\rho(t)= \pm e^{\xi_{\lambda}\left(t, \theta_{0}\right)} \cdot e^{-i \pi \eta_{\lambda}(t)} \cdot e^{-\frac{i \pi}{2} \sum_{j=0}^{d}(-1)^{j} j d_{j, \lambda}} \cdot \rho_{\Gamma_{t,[0, \lambda]}} .
$$

Lemma 9.2 Under the above assumptions, the product

$$
e^{\xi_{\lambda}\left(t, \theta_{0}\right)} \cdot \rho_{\Gamma_{t,[0, \lambda]}} \in \operatorname{Det}\left(H^{\bullet}(M, F)\right)
$$

is independent of $t \in\left(t_{0}-\delta, t_{0}+\delta\right)$.

Proof Recall that we have chosen $\lambda \geq 0$ and $\delta>0$ so that there are no eigenvalues of $\mathcal{B}(t)^{2}$ with absolute value $\lambda$ for any $t \in\left(t_{0}-\delta, t_{0}+\delta\right)$.

We shall use the following notation (cf, for example, [11, Section 2]): Suppose $f(s)$ is a function of a complex parameter $s$ which is meromorphic near $s=0$. We call the zero order term in the Laurent expansion of $f$ near $s=0$ the finite part of $f$ at 0 and denote it by F. p.s=0 $f(s)$. A verbatim repetition of the proof of [9, formula (9.13)] shows that

$$
\frac{d}{d t} \xi_{\lambda}\left(t, \theta_{0}\right)=\frac{1}{2} \sum_{j=0}^{d}(-1)^{j} \mathrm{~F} \cdot \mathrm{p} \cdot{ }_{\mathrm{s}=0} \operatorname{Tr}\left[\left.\dot{\Gamma}_{t} \Gamma_{t}\left(\left(\mathcal{B}^{(\lambda, \infty)}(t)\right)^{2}\right)_{2 \theta_{0}}^{-s}\right|_{\Omega_{(\lambda, \infty)}^{j}(M, E)}\right]
$$

Since $\mathcal{B}^{2}(t)$ is an elliptic differential operator, the dimension of $\Omega_{[0, \lambda]}^{\bullet}(M, E)$ is finite. Let $\varepsilon \neq 0$ be a small enough real number so that $\mathcal{B}(t)^{2}+\varepsilon$ is bijective and $2 \theta_{0}$ is an Agmon angle for $\left(\mathcal{B}^{(\lambda, \infty)}(t)\right)^{2}+\varepsilon$. Then, for each $j=0, \ldots, d$, we have

$$
\begin{aligned}
& \text { F.p.s }{ }_{\mathrm{s}=0} \operatorname{Tr}\left[\left.\dot{\Gamma}_{t} \Gamma_{t}\left(\left(\mathcal{B}^{(\lambda, \infty)}(t)\right)^{2}\right)_{2 \theta_{0}}^{-s}\right|_{\Omega_{(\lambda, \infty)}^{j}(M, E)}\right] \\
&= \text { F.p.s } \cdot \mathrm{s}=0 \\
& \operatorname{Tr}\left[\left.\dot{\Gamma}_{t} \Gamma_{t}\left(\left(\mathcal{B}^{(\lambda, \infty)}(t)\right)^{2}+\varepsilon\right)_{2 \theta_{0}}^{-s}\right|_{\Omega^{j}(M, E)}\right]-\operatorname{Tr}\left[\left.\dot{\Gamma}_{t} \Gamma_{t}\right|_{\Omega_{[0, \lambda]}^{j}(M, E)}\right] .
\end{aligned}
$$

By a slight generalization of a result of Seeley [31], which is discussed in Ray-Singer [29] and, in greater generality, in Grubb-Seeley [20], the first summand on the right hand side of (9-7) is given by a local formula ie by the integral $\int_{M} \phi_{t}$ of a differential form $\phi_{t}$, whose value at any point $x \in M$ depends only on the values of the components of the metric tensor $g_{t}^{M}$ and a finite number of their derivatives at $x$. Moreover, since the dimension of the manifold $M$ is odd, the differential form $\phi_{t}$ vanishes identically. Thus we obtain from (9-6) and (9-7)

$$
\frac{d}{d t} \xi_{\lambda}\left(t, \theta_{0}\right)=-\frac{1}{2} \sum_{j=0}^{d}(-1)^{j} \operatorname{Tr}\left[\left.\dot{\Gamma}_{t} \Gamma_{t}\right|_{\Omega_{[0, \lambda]}^{j}(M, E)}\right] .
$$


Combining this equation with Proposition 4.9, we get

$$
\frac{d}{d t} e^{\xi_{\lambda}\left(t, \theta_{0}\right)} \cdot \rho_{\Gamma_{t,[0, \lambda]}}=0 .
$$

\subsection{Dependence of the $\eta$-invariant on the metric}

From (9-5) and Lemma 9.2 we see that the dependence of $\rho(t)$ on $t \in\left(t_{0}-\delta, t_{0}+\delta\right)$ is determined up to a sign by the dependence of $\eta_{\lambda}(t)$ on $t$.

Lemma 9.4 For any $t_{1}, t_{2} \in\left(t_{0}-\delta, t_{0}+\delta\right)$ we have

$$
\eta_{\lambda}\left(t_{1}\right)-\eta_{\lambda}\left(t_{2}\right) \equiv \eta\left(\mathcal{B}_{\text {even }}\left(t_{1}\right)\right)-\eta\left(\mathcal{B}_{\text {even }}\left(t_{2}\right)\right), \quad \bmod \mathbb{Z}
$$

Proof Recall that $\Omega_{[0, \lambda], t}^{\bullet}(M, E)$ denotes the span of the generalized eigenvectors of $\mathcal{B}(t)^{2}$ corresponding to eigenvalues with absolute value $\leq \lambda$ and that $\lambda$ and $\delta$ were chosen so that

$$
\operatorname{dim} \Omega_{[0, \lambda], t}^{j}(M, E)=\mathrm{const}, \quad t \in\left(t_{0}-\delta, t_{0}+\delta\right), j=0, \ldots, d .
$$

Since the dimension of the space $\Omega_{[0, \lambda], t}^{\text {even }}(M, E)$ is finite, formula (6-34) says that $\eta\left(0, \mathcal{B}_{\text {even }}^{[0, \lambda]}(t)\right)$ is equal to the sum of the algebraic multiplicities of the eigenvalues of $\mathcal{B}_{\text {even }}^{[0, \lambda]}(t)$ with positive real parts minus the sum of the algebraic multiplicities of the eigenvalues of $\mathcal{B}_{\text {even }}^{[0, \lambda]}(t)$ with negative real parts. It then follows from (6-35) that

$$
\eta\left(\mathcal{B}_{\text {even }}^{[0, \lambda]}(t)\right) \equiv \frac{1}{2} \operatorname{dim} \Omega_{[0, \lambda], t}^{\text {even }}(M, E), \quad \bmod \mathbb{Z} .
$$

By the definition of the $\eta$-invariant,

$$
\eta\left(\mathcal{B}_{\text {even }}(t)\right)-\eta_{\lambda}(t)=\eta\left(\mathcal{B}_{\text {even }}^{[0, \lambda]}(t)\right) .
$$

Hence, from (9-10) and (9-11), we conclude that, modulo $\mathbb{Z}$,

$$
\eta\left(\mathcal{B}_{\text {even }}(t)\right)-\eta_{\lambda}(t) \equiv \text { const }
$$

for $t \in\left(t_{0}-\delta, t_{0}+\delta\right)$.

We now need to study the dependence of $\eta\left(\mathcal{B}_{\text {even }}\left(\nabla, g^{M}\right)\right)$ on the Riemannian metric $g^{M}$. Fortunately, this was essentially done in [2] and [19]. Below we present a brief review of the relevant results.

Let $\mathcal{B}_{\text {trivial }}=\mathcal{B}_{\text {trivial }}\left(g^{M}\right): \Omega^{\text {even }}(M) \rightarrow \Omega^{\text {even }}(M)$ denote the even part of the odd signature operator corresponding to the metric $g^{M}$ and the trivial line bundle over $M$ endowed with the trivial connection. Set

$$
\eta_{\text {trivial }}=\eta_{\text {trivial }}\left(g^{M}\right):=\frac{1}{2} \eta\left(0, B_{\text {trivial }}\left(g^{M}\right)\right) .
$$


Since the operator $\mathcal{B}_{\text {trivial }}$ is self-adjoint, $\eta_{\text {trivial }}$ is a real number. Moreover, since all the eigenvalues of $\mathcal{B}_{\text {trivial }}$ are real, it follows from (6-24) that

$$
\eta_{\text {trivial }}=\eta\left(\mathcal{B}_{\text {trivial }}\right)-\frac{1}{2} m_{0}\left(\mathcal{B}_{\text {trivial }}\right)=\eta\left(\mathcal{B}_{\text {trivial }}\right)-\frac{1}{2} \sum_{p=0}^{r-1} \operatorname{dim} H^{2 p}(M, \mathbb{C}) .
$$

Also, if $\operatorname{dim} M \equiv 1(\bmod 4)$, then $\eta_{\text {trivial }}=0, \operatorname{cf}[1]$.

It is shown in [19, page 52] (see also [2, Theorem 2.4] where the case of unitary connection is established) that modulo $\mathbb{Z}$ the difference

$$
\eta\left(\mathcal{B}_{\text {even }}\left(\nabla, g^{M}\right)\right)-(\operatorname{rank} E) \eta\left(\mathcal{B}_{\text {trivial }}\left(g^{M}\right)\right)
$$

is independent of the Riemannian metric.

\subsection{Removing the metric anomaly}

We are now ready to state the main result of this section.

Theorem 9.6 Let $E$ be a flat vector bundle over a closed oriented odd-dimensional manifold $M$. Let $N$ be an oriented manifold whose oriented boundary is the disjoint union of two copies of $M$. The element

$$
\rho\left(\nabla, g^{M}\right) \cdot e^{i \pi(\operatorname{rank} E) \eta_{\text {trivial }}\left(g^{M}\right)} \in \operatorname{Det}\left(H^{\bullet}(M, E)\right),
$$

where $\rho\left(\nabla, g^{M}\right) \in \operatorname{Det}\left(H^{\bullet}(M, E)\right)$ is defined in (7-15), is independent of $g^{M}$.

In particular, if $\operatorname{dim} M \equiv 1(\bmod 4)$, then $\eta_{\text {trivial }}=0$ and, hence, $\rho\left(\nabla, g^{M}\right)$ is independent of $g^{M}$.

Proof Let $g_{t}^{M},(t \in \mathbb{R})$ be a family of Riemannian metrics on $M$. We shall use the notation of Section 9.1. From (9-5) we obtain for $t \in\left(t_{0}-\delta, t_{0}+\delta\right)$

$$
\begin{aligned}
& \rho(t) \cdot e^{i \pi(\operatorname{rank} E) \eta_{\text {trivial }}\left(g^{M}\right)}= \\
& \quad \pm e^{\xi_{\lambda}(t)} \cdot e^{-i \pi \eta_{\lambda}(t)} \cdot e^{-\frac{i \pi}{2} \sum_{j=0}^{d}(-1)^{j} j d_{j, \lambda}} \cdot \rho_{\Gamma_{t,[0, \lambda]}} \cdot e^{i \pi(\operatorname{rank} E) \eta_{\text {trivial }}\left(g^{M}\right)} .
\end{aligned}
$$

Combining this latter equality with Lemma 9.2 and the metric independence of the reduction of (9-16) modulo $\mathbb{Z}$ we conclude that for any $t_{1}, t_{2} \in(t-\delta, t+\delta)$

$$
\rho\left(t_{1}\right) \cdot e^{i \pi(\operatorname{rank} E) \eta_{\text {trivial }}\left(g^{M}\right)}= \pm \rho\left(t_{2}\right) \cdot e^{i \pi(\operatorname{rank} E) \eta_{\text {trivial }}\left(g^{M}\right)}
$$

Since the function $t \mapsto \rho(t) \cdot e^{i \pi(\operatorname{rank} E) \eta_{\text {trivial }}\left(g^{M}\right)}$ is continuous and nonzero the sign in the right hand side of $(9-15)$ must be positive. Hence, the theorem is proven. 


\subsection{The definition of the refined analytic torsion}

We now arrive to the main definition of the paper.

Definition 9.8 Let $E$ be a complex vector bundle over a closed, oriented, odddimensional manifold $M$ and let $\nabla$ be the flat connection on $E$. The refined analytic torsion $\rho_{\mathrm{an}}=\rho_{\mathrm{an}}(\nabla)$ is the element of $\operatorname{Det}\left(H^{\bullet}(M, E)\right)$ defined by (9-14).

It follows from Theorem 9.6 that the refined analytic torsion is independent of any choices made in its definition, ie, it is a differential invariant of the flat vector bundle $(E, \nabla)$.

Remark 9.9 Since $\operatorname{dim} M$ is odd, there exists an oriented manifold $N$ whose oriented boundary is the disjoint union of two copies of $M$ (with the same orientation), cf Wall [38], Rudyak [30, Theorem IV.6.5]. Then, cf [9, Section 11], the signature theorem for manifolds with boundary implies that, modulo $\mathbb{Z}$,

$$
\eta_{\lambda}(t)-\frac{1}{2} \operatorname{rank} E \int_{N} L\left(p, g_{t}^{M}\right)
$$

where $L\left(p, g^{M}\right)$ is the Hirzebruch $L$-polynomial in the Pontrjagin forms of any Riemannian metric on $N^{\prime}$ which near $M$ is the product of $g^{M}$ and the standard metric on the half-line, is independent of $t \in\left(t_{0}-\delta, t_{0}+\delta\right)$. The same arguments as in the proof of Theorem 9.6 show that the element

$$
\rho_{\mathrm{an}}^{\prime}(\nabla):=\rho\left(\nabla, g^{M}\right) \cdot \exp \left(i \pi \cdot \frac{1}{2} \operatorname{rank} E \int_{N} L\left(p, g^{M}\right)\right)
$$

is independent of the Riemannian metric $g^{M}$. It can be used as an alternative definition of the refined analytic torsion. It has an advantage that the second factor in (9-17) is much easier to compute than the second factor in (9-14). Note, however, that $\rho^{\prime}(\nabla)$ does depend on the choice of the manifold $N$. However, it is easy to see that $\rho_{\mathrm{an}}^{\prime}(\nabla)$ is defined up to multiplication by $i^{k \cdot \operatorname{rank} E}(k \in \mathbb{Z})$. If $\operatorname{rank} E$ is even then $\rho_{\mathrm{an}}^{\prime}(\nabla)$ is well defined up to a sign, and if rank $E$ is divisible by 4 , then $\rho_{\text {an }}(\nabla)$ is a well defined element of $\operatorname{Det}\left(H^{\bullet}(M, E)\right)$.

(Here a quantity being well defined means that it depends only on $M, E$ and $\nabla$.)

\section{A duality theorem for the refined analytic torsion}

In this section we establish a relationship between the refined analytic torsion corresponding to a flat connection and that of its dual. This result is used in the next section in order to calculate the Ray-Singer norm of the refined analytic torsion, but it is also of independent interest. 


\subsection{The dual connection}

Suppose $M$ is a closed oriented manifold of odd dimension $d=2 r-1$. Let $E \rightarrow M$ be a complex vector bundle over $M$ and let $\nabla$ be a flat connection on $E$. Fix a Hermitian metric $h^{E}$ on $E$. Denote by $\nabla^{\prime}$ the connection on $E$ dual to the connection $\nabla$. It is defined by the formula

$$
d h^{E}(u, v)=h^{E}(\nabla u, v)+h^{E}\left(u, \nabla^{\prime} v\right), \quad u, v \in C^{\infty}(M, E) .
$$

For $\omega_{1}, \omega_{2} \in \Omega^{\bullet}(M, E)$ of the form $\omega_{i}=s_{i} \otimes \chi_{i}$ with $s_{i} \in C^{\infty}(M, E), \chi_{i} \in \Omega^{\bullet}(M, \mathbb{R})$, define

$$
h^{E}\left(\left(s_{1} \otimes \chi_{1}\right) \wedge\left(s_{2} \otimes \chi_{2}\right)\right):=h^{E}\left(s_{1}, s_{2}\right) \cdot \chi_{1} \wedge \chi_{2} .
$$

Then $h^{E}$ extends in a canonical way to a sesquilinear map

$$
h^{E}: \Omega^{\bullet}(M, E) \times \Omega^{\bullet}(M, E) \longrightarrow \Omega^{\bullet}(M, \mathbb{C}) .
$$

For each $j=0, \ldots, d$, we then obtain a sesquilinear pairing

$$
\Omega^{j}(M, E) \times \Omega^{d-j}(M, E) \longrightarrow \mathbb{C}, \quad\left(\omega_{1}, \omega_{2}\right) \mapsto \int_{M} h^{E}\left(\omega_{1} \wedge \omega_{2}\right) .
$$

We denote by $E^{\prime}$ the flat vector bundle $\left(E, \nabla^{\prime}\right)$, referring to $E^{\prime}$ as the dual of the flat vector bundle $E$. The pairing (10-3) induces a non-degenerate sesquilinear pairing

$$
H^{j}\left(M, E^{\prime}\right) \otimes H^{d-j}(M, E) \longrightarrow \mathbb{C}, \quad j=0, \ldots, d,
$$

and, hence, identifies $H^{j}\left(M, E^{\prime}\right)$ with the dual space of $H^{d-j}(M, E)$. Using the construction of Section 3.4 with $\tau: \mathbb{C} \rightarrow \mathbb{C}$ being the complex conjugation) we thus obtain an anti-linear isomorphism

$$
\alpha: \operatorname{Det}\left(H^{\bullet}(M, E)\right) \longrightarrow \operatorname{Det}\left(H^{\bullet}\left(M, E^{\prime}\right)\right) .
$$

\subsection{The duality theorem}

Fix a compact oriented manifold $N$ whose boundary is diffeomorphic to two disjoint copies of $M$. From (9-13), we conclude that the number

$$
\exp \left(2 i \pi\left(\bar{\eta}\left(\nabla, g^{M}\right)-(\operatorname{rank} E) \eta_{\text {trivial }}\left(g^{M}\right)\right)\right)
$$

where $\bar{\eta}$ denotes the complex conjugate of $\eta$, is independent of the choice of the Riemannian metric $g^{M}$.

The main result of this section is the following duality theorem. 
Theorem 10.3 Let $E \rightarrow M$ be a complex vector bundle over a closed oriented odddimensional manifold $M$ and let $\nabla$ be a flat connection on $E$. Let $\nabla^{\prime}$ denote the connection dual to $\nabla$ with respect to a Hermitian metric $h^{E}$ on $E$. Let $N$ be a compact oriented manifold whose boundary is diffeomorphic to two disjoint copies of $M$. Then

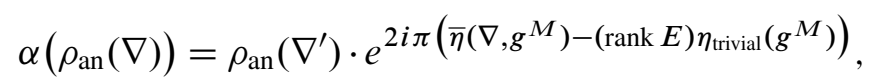

where $\alpha$ is the anti-linear isomorphism (10-4), $g^{M}$ is any Riemannian metric on $M$, and $L\left(p, g^{M}\right)$ is the Hirzebruch $L$-polynomial in the Pontrjagin forms of any Riemannian metric on $N$ which near $M$ is the product of $g^{M}$ and the standard metric on the half-line.

In particular, if $\operatorname{dim} M \equiv 1(\bmod 4)$, then

$$
\alpha\left(\rho_{\mathrm{an}}(\nabla)\right)=\rho_{\mathrm{an}}\left(\nabla^{\prime}\right) \cdot e^{2 i \pi \bar{\eta}\left(\nabla, g^{M}\right)} .
$$

The rest of this section is concerned with the proof of Theorem 10.3.

\subsection{Choices of the metric and the spectral cut}

Till the end of this section we fix a Riemannian metric $g^{M}$ on $M$ and set $\mathcal{B}=\mathcal{B}\left(\nabla, g^{M}\right)$ and $\mathcal{B}^{\prime}=\mathcal{B}\left(\nabla^{\prime}, g^{M}\right)$. We also fix an Agmon angle $\theta \in(-\pi / 2,0)$ for the odd signature operator $\mathcal{B}=\mathcal{B}\left(\nabla, g^{M}\right)$ such that there are no eigenvalues of $\mathcal{B}$ in the solid angles $L_{[-\theta-\pi,-\pi / 2]}, L_{(-\pi / 2, \theta]}, L_{[-\theta, \pi / 2)}$, and $L_{(\pi / 2, \theta+\pi]}$.

Let $\mathcal{B}^{\prime}$ denote the odd signature operator associated to the connection $\nabla^{\prime}$ and the metric $g^{M}$. One easily checks, cf [3, Proposition 3.58], that

$$
\nabla^{*}=\Gamma \nabla^{\prime} \Gamma \text { and }\left(\nabla^{\prime}\right)^{*}=\Gamma \nabla \Gamma \text {. }
$$

Using (7-2), (10-8), and the equality $\Gamma^{*}=\Gamma$ (cf [3, Proposition 3.58]), one readily sees that the adjoint $\mathcal{B}^{*}$ of $\mathcal{B}$ satisfies

$$
\mathcal{B}^{*}=\mathcal{B}^{\prime}
$$

Our choice of the angle $\theta$ guarantees that $\pm 2 \theta$ are Agmon angles for the operator

$$
\left(\Gamma \nabla^{\prime}\right)^{2}=\left((\Gamma \nabla)^{2}\right)^{*} \text {. }
$$

In particular, for each $\lambda \geq 0$, the number $\xi_{\lambda}\left(\nabla^{\prime}, g^{M}, \theta\right)$ can be defined by formula (8-2), with the same angle $\theta$ and with $\nabla$ replaced by $\nabla^{\prime}$ everywhere. 


\subsection{A choice of $\lambda$}

Since the leading symbol of $\mathcal{B}$ is self-adjoint, there are at most finitely many purely imaginary eigenvalues of $\mathcal{B}$. Hence, there exists $\lambda \geq 0$ such that there are no purely imaginary eigenvalues of $\mathcal{B}$ with absolute value $\geq \sqrt{\lambda}$. In other words, the operator $\mathcal{B}^{(\lambda, \infty)}$ does not have purely imaginary eigenvalues. Moreover, our assumptions on $\theta$ in Section 10.4 imply that no eigenvalue of $\mathcal{B}^{(\lambda, \infty)}$ lies in the solid angles $L_{[-\theta-\pi, \theta]}$ and $L_{[-\theta, \theta+\pi]}$. It follows that no eigenvalue of the operator $\left(\mathcal{B}^{(\lambda, \infty)}\right)^{2}$ lies in the solid angle $L_{[-2 \theta, 2 \theta+2 \pi]}$.

Lemma 10.6 Let $\theta$ be as in Section 10.4 and let $\lambda \geq 0$ be big enough so that the operator $\mathcal{B}^{(\lambda, \infty)}$ does not have purely imaginary eigenvalues. Then

$$
\xi_{\lambda}\left(\nabla^{\prime}, g^{M}, \theta\right)=\bar{\xi}_{\lambda}\left(\nabla, g^{M}, \theta\right),
$$

and

$$
\eta_{\lambda}\left(\nabla^{\prime}, g^{M}\right)=\bar{\eta}_{\lambda}\left(\nabla, g^{M}\right),
$$

where $\bar{z}$ denotes the complex conjugate of the number $z \in \mathbb{C}$.

Proof Let $\mathcal{B}^{\prime}(\lambda, \infty)$ denote the restriction of $\mathcal{B}^{\prime}$ to the span of the generalized eigenvectors of $\left(\mathcal{B}^{\prime}\right)^{2}$ corresponding to the eigenvalues whose absolute values are greater than $\lambda$. From (10-9), we obtain

$$
\left(\mathcal{B}^{\prime(\lambda, \infty)}\right)^{*}=\mathcal{B}^{(\lambda, \infty)} .
$$

Hence, with our assumptions on $\theta$, we have, for any $j=0, \ldots, d$,

$$
\begin{aligned}
\operatorname{LDet}_{2 \theta}^{\prime}\left(\left.\left(\mathcal{B}^{\prime(\lambda, \infty)}\right)^{2}\right|_{\Omega_{(\lambda, \infty)}^{j}(M, E)}\right) & \overline{\operatorname{LDet}_{-2 \theta}^{\prime}\left(\left.\left(\left(\mathcal{B}^{\prime(\lambda, \infty)}\right)^{*}\right)^{2}\right|_{\Omega_{(\lambda, \infty)}^{j}(M, E)}\right)} \\
= & \left.\overline{\operatorname{LDet}_{-2 \theta}^{\prime}\left(\left.\left(\mathcal{B}^{(\lambda, \infty)}\right)^{2}\right|_{\Omega_{(\lambda, \infty)}^{j}}(M, E)\right.}\right) .
\end{aligned}
$$

Since there are no eigenvalues of $\left(\mathcal{B}^{(\lambda, \infty)}\right)^{2}$ in the solid angle $L_{[-2 \theta, 2 \theta+2 \pi]}$,

$$
\operatorname{LDet}_{-2 \theta}^{\prime}\left(\left.\left(\mathcal{B}^{(\lambda, \infty)}\right)^{2}\right|_{\Omega_{(\lambda, \infty)}^{j}(M, E)}\right)=\operatorname{LDet}_{2 \theta}^{\prime}\left(\left.\left(\mathcal{B}^{(\lambda, \infty)}\right)^{2}\right|_{\Omega_{(\lambda, \infty)}^{j}(M, E)}\right) .
$$

The equality (10-10) follows now from (10-13) and the definition (8-3) of $\xi_{\lambda}\left(\nabla, g^{M}, \theta\right)$.

Let $\Pi_{>}\left(\right.$resp. $\left.\Pi_{<}\right)$denote the spectral projection of $\mathcal{B}$ onto the span of all generalized eigenvectors of $\mathcal{B}$ corresponding to eigenvalues with positive (resp. negative) real part. 
Let $Q$ denote the spectral projection of $\mathcal{B}$ onto the span of all generalized eigenvectors of $\mathcal{B}$ corresponding to eigenvalues whose absolute value is larger than $\lambda$. Let $\Pi_{>}^{\prime}$, $\Pi_{<}^{\prime}$, and $Q^{\prime}$ be similarly defined spectral projections of $\mathcal{B}^{\prime}$. Then, since the operators $\mathcal{B}^{(\lambda, \infty)}$ and $\mathcal{B}^{\prime(\lambda, \infty)}$ have no purely imaginary eigenvalues, we conclude from (6-34) and (6-35) that

$$
\begin{aligned}
2 \eta_{\lambda}\left(\nabla, g^{M}\right) & =\zeta_{\theta}\left(0, Q \Pi_{>}, \mathcal{B}_{\text {even }}\right)-\zeta_{\theta}\left(0, Q \Pi_{<}, \mathcal{B}_{\text {even }}\right) \\
2 \eta_{\lambda}\left(\nabla^{\prime}, g^{M}\right) & =\zeta_{\theta}\left(0, Q^{\prime} \Pi_{>}^{\prime}, \mathcal{B}_{\text {even }}^{\prime}\right)-\zeta_{\theta}\left(0, Q^{\prime} \Pi_{<}^{\prime}, \mathcal{B}_{\text {even }}^{\prime}\right) .
\end{aligned}
$$

From (10-12) and our assumptions on $\theta$, we obtain

$$
\begin{aligned}
& \zeta_{\theta}\left(s, Q^{\prime} \Pi_{>}^{\prime}, \mathcal{B}_{\text {even }}^{\prime}\right)=\bar{\zeta}_{\theta}\left(\bar{s}, Q \Pi_{>}, \mathcal{B}_{\text {even }}\right), \\
& \zeta_{\theta}\left(s, Q^{\prime} \Pi_{<}^{\prime}, \mathcal{B}_{\text {even }}^{\prime}\right)=e^{-2 \pi i s} \cdot \bar{\zeta}_{\theta}\left(\bar{s}, Q \Pi_{<}, \mathcal{B}_{\text {even }}\right) .
\end{aligned}
$$

The equality (10-11) follows immediately from (10-14) and (10-15).

\subsection{Small eigenvalues of $\mathcal{B}$ and $\mathcal{B}^{\prime}$}

Let $\Omega_{[0, \lambda]}^{\bullet}\left(M, E^{\prime}\right) \subset \Omega^{\bullet}(M, E)$ denote the span of the eigenvectors of $\left(\mathcal{B}^{\prime}\right)^{2}$ corresponding to the eigenvalues with absolute value $\leq \lambda$. Then $\Omega_{[0, \lambda]}^{\bullet}\left(M, E^{\prime}\right)$ is a subcomplex of $\left(\Omega^{\bullet}(M, E), \nabla^{\prime}\right)$ preserved by the chirality operator $\Gamma$.

The pairing (10-3) defines a non-degenerate sesquilinear pairing

$$
\Omega_{[0, \lambda]}^{j}\left(M, E^{\prime}\right) \times \Omega_{[0, \lambda]}^{d-j}(M, E) \longrightarrow \mathbb{C},
$$

and, hence, identifies $\Omega_{[0, \lambda]}^{\bullet}\left(M, E^{\prime}\right)$ with the dual complex of $\Omega_{[0, \lambda]}^{\bullet}(M, E)$.

As in Section 8, set, for $j=0, \ldots, d$,

$$
d_{j, \lambda}=\operatorname{dim} \Omega_{[0, \lambda]}^{j}(M, E), \quad d_{j, \lambda}^{\prime}=\operatorname{dim} \Omega_{[0, \lambda]}^{j}\left(M, E^{\prime}\right) .
$$

Since $\Gamma^{2}=1$, we obtain from (7-2), $\mathcal{B}_{j}=\Gamma \circ \mathcal{B}_{d-j} \circ \Gamma$ and, hence,

$$
\Gamma\left(\Omega_{[0, \lambda]}^{j}(M, E)\right)=\Omega_{[0, \lambda]}^{d-j}(M, E), \quad j=0, \ldots, d .
$$

Therefore

$$
d_{j, \lambda}=d_{d-j, \lambda}, \quad j=0, \ldots, d .
$$

Hence,

(10-18) $\sum_{j=0}^{d}(-1)^{j} j d_{j, \lambda}=\sum_{p=0}^{r-1}(2 p-(d-2 p)) d_{2 p, \lambda}=4 \sum_{p=0}^{r-1} p d_{2 p, \lambda}-d \sum_{p=0}^{r-1} d_{2 p, \lambda}$. 
In particular,

$$
\sum_{j=0}^{d}(-1)^{j} j d_{j, \lambda} \equiv \sum_{p=0}^{r-1} d_{2 p, \lambda}=\operatorname{dim} \Omega_{[0, \lambda]}^{\mathrm{even}}(M, E), \quad \bmod 2 \mathbb{Z} .
$$

From (10-9), we conclude that $d_{j, \lambda}=d_{d-j, \lambda}^{\prime}(j=0, \ldots, d)$. Combining this equality with (10-17), we get $d_{j, \lambda}=d_{j, \lambda}^{\prime}$. Hence, by (10-19),

$$
\sum_{j=0}^{d}(-1)^{j} j d_{j, \lambda}^{\prime} \equiv \operatorname{dim} \Omega_{[0, \lambda]}^{\mathrm{even}}(M, E), \quad \bmod 2 \mathbb{Z} .
$$

From (8-1), (9-11), and (10-20), we obtain, modulo $2 \mathbb{Z}$,

$$
\begin{aligned}
2 \eta\left(\mathcal{B}_{\text {even }}\left(\nabla, g^{M}\right)\right) & =2 \eta\left(\mathcal{B}_{\text {even }}^{(\lambda, \infty)}\left(\nabla, g^{M}\right)\right)+2 \eta\left(\mathcal{B}_{\text {even }}^{[0, \lambda]}\left(\nabla, g^{M}\right)\right) \\
& \equiv 2 \eta_{\lambda}\left(\nabla, g^{M}\right)+\sum_{j=0}^{d}(-1)^{j} j d_{j, \lambda} .
\end{aligned}
$$

Similarly,

$$
2 \eta\left(\mathcal{B}_{\text {even }}\left(\nabla^{\prime}, g^{M}\right)\right) \equiv 2 \eta_{\lambda}\left(\nabla^{\prime}, g^{M}\right)+\sum_{j=0}^{d}(-1)^{j} j d_{j, \lambda}, \quad \bmod 2 \mathbb{Z}
$$

Proof of Theorem 10.3 Let $\rho_{\Gamma_{[0, \lambda]}}^{\prime}$ be the refined torsion of the complex $\Omega_{[0, \lambda]}^{\bullet}\left(M, E^{\prime}\right)$ associated to the restriction of $\Gamma$ to $\Omega_{[0, \lambda]}^{\bullet}\left(M, E^{\prime}\right)$.

Since $\Gamma^{*}=\Gamma(\operatorname{cf}[3$, Proposition 3.58]), we obtain from Lemma 4.11 and the definition $(10-4)$ of $\alpha$,

$$
\rho_{\Gamma_{[0, \lambda]}}^{\prime}=\alpha\left(\rho_{\Gamma_{[0, \lambda]}}\right) .
$$

From (7-15), (8-5), and Definition 9.8, we obtain

$$
\begin{array}{r}
\rho_{\mathrm{an}}(\nabla)=\rho_{\Gamma_{[0, \lambda]}} \cdot \exp \left(\xi_{\lambda}\left(\nabla, g^{M}, \theta\right)-i \pi \eta_{\lambda}\left(\nabla, g^{M}\right)\right. \\
\left.-\frac{i \pi}{2} \sum_{j=0}^{d}(-1)^{j} j d_{j, \lambda}+i \pi(\operatorname{rank} E) \eta_{\text {trivial }}\left(g^{M}\right)\right) .
\end{array}
$$


Since $\alpha$ is an anti-linear isomorphism, $\alpha\left(\rho_{\mathrm{an}} \cdot z\right)=\alpha\left(\rho_{\mathrm{an}}\right) \cdot \bar{z}$ for any $z \in \mathbb{C}$. Hence, from (10-22) and (10-23) we get

$$
\begin{aligned}
\alpha\left(\rho_{\mathrm{an}}(\nabla)\right)=\rho_{\Gamma_{[0, \lambda]}^{\prime}}^{\prime} \cdot \exp \left(\bar{\xi}_{\lambda}\left(\nabla, g^{M}, \theta\right)+i \pi \bar{\eta}_{\lambda}\left(\nabla, g^{M}\right)\right. \\
\left.+\frac{i \pi}{2} \sum_{j=0}^{d}(-1)^{j} j d_{j, \lambda}-i \pi(\operatorname{rank} E) \eta_{\text {trivial }}\left(g^{M}\right)\right) .
\end{aligned}
$$

Using Lemma 10.6 and the analogue of (10-23) for $\rho_{\mathrm{an}}\left(\nabla^{\prime}\right)$, we obtain from (10-24)

$$
\begin{aligned}
\alpha\left(\rho_{\mathrm{an}}(\nabla)\right)=\rho_{\mathrm{an}}\left(\nabla^{\prime}\right) \cdot \exp & \left(2 i \pi \bar{\eta}_{\lambda}\left(\nabla, g^{M}\right)\right. \\
+ & \left.i \pi \sum_{j=0}^{d}(-1)^{j} j d_{j, \lambda}-i \pi(\operatorname{rank} E) \eta_{\text {trivial }}\left(g^{M}\right)\right) .
\end{aligned}
$$

From (10-25) and (10-21) we obtain (10-6).

\section{Comparison with the Ray-Singer torsion}

In this section we calculate the Ray-Singer norm $\left\|\rho_{\mathrm{an}}\right\|_{\operatorname{Det}(H \bullet(M, E))}^{\mathrm{RS}}$ of the refined analytic torsion. In particular, we show that, if $\nabla$ is a Hermitian connection, then $\left\|\rho_{\mathrm{an}}\right\|_{\operatorname{Det}\left(H^{\bullet}(M, E)\right)}^{\mathrm{RS}}=1$.

\subsection{The Ray-Singer torsion}

Let $E \rightarrow M$ be a complex vector bundle over a closed oriented manifold $M$ of odd dimension $d=2 r-1$ and let $\nabla$ be a flat connection on $E$. Fix a Riemannian metric $g^{M}$ on $M$ and a Hermitian metric $h^{E}$ on $E$. Let $\nabla^{*}$ denote the adjoint of $\nabla$ with respect to the scalar product $\langle\cdot, \cdot\rangle$ on $\Omega^{\bullet}(M, E)$ defined by $h^{E}$ and the Riemannian metric $g^{M}$. Let

$$
\Delta=\nabla^{*} \nabla+\nabla \nabla^{*}
$$

be the Laplacian. We denote by $\Delta_{k}$ the restriction of $\Delta$ to $\Omega^{k}(M, E)$.

The Ray-Singer torsion $T^{\mathrm{RS}}$ of $E,[29 ; 4 ; 11]$, is the positive real number defined by 5

$$
T^{\mathrm{RS}}=T^{\mathrm{RS}}(\nabla):=\exp \left(\frac{1}{2} \sum_{k=0}^{d}(-1)^{k} k \operatorname{LDet}_{-\pi}^{\prime}\left(\Delta_{k}\right)\right) .
$$

\footnotetext{
${ }^{5}$ Our sign convention is different from [29], [11], and [9] but is consistent with [4]. In our notations, the torsion defined in $[29 ; 11 ; 9]$ is equal to $1 / T^{\mathrm{RS}}$.
} 
Note that $\Delta_{k}$ is a self-adjoint, non-negative operator. Therefore, all its eigenvalues are non-negative and $\operatorname{LDet}_{-\pi}^{\prime}\left(\Delta_{k}\right)$ is well defined.

More generally, suppose $\mathcal{I}$ is an interval of the form $[0, \lambda],(\lambda, \mu]$, or $(\lambda, \infty](\mu \geq \lambda \geq 0)$ and let $\Pi_{\Delta_{k}, \mathcal{I}}$ be the spectral projection of $\Delta$ corresponding to $\mathcal{I}$, cf Section 6.10. Set

$$
\check{\Omega}_{\mathcal{I}}^{k}(M, E):=\Pi_{\Delta_{k}, \mathcal{I}}\left(\Omega^{\bullet}(M, E)\right) \subset \check{\Omega}^{\bullet}(M, E) .
$$

Let $\Delta_{k}^{\mathcal{I}}$ denote the restriction of $\Delta_{k}$ to $\check{\Omega}_{\mathcal{I}}^{k}(M, E)$ and define

$$
\left.T_{\mathcal{I}}^{\mathrm{RS}}=T_{\mathcal{I}}^{\mathrm{RS}}(\nabla):=\exp \left(\frac{1}{2} \sum_{k=0}^{d}(-1)^{k} k \operatorname{LDet}_{-\pi}^{\prime}\left(\Delta_{k}^{\mathcal{I}}\right)\right)\right) .
$$

In particular, by (11-2), $T^{\mathrm{RS}}=T_{(0, \infty)}^{\mathrm{RS}}$. By (6-15), for any non-negative, real numbers $\mu \geq \lambda \geq 0$,

$$
T_{(\lambda, \infty)}^{\mathrm{RS}}=T_{(\lambda, \mu]}^{\mathrm{RS}} \cdot T_{(\mu, \infty]}^{\mathrm{RS}} \cdot
$$

\subsection{The Ray-Singer metric on the determinant line of cohomology}

If the connection $\nabla$ is acyclic, that is, if the cohomology $H^{\bullet}(M, E)$ vanishes, then the Ray-Singer torsion is independent of the Hermitian metric $h^{E}$ and the Riemannian metric $g^{M}$, cf [29; 4]. If the cohomology does not vanish, then $T^{\mathrm{RS}}$, in general, depends on the choice of the two metrics. To construct a metric independent invariant of the flat vector bundle $E$ one needs to take into account the contribution of the space of harmonic forms. An elegant way to do this, which was proposed by Quillen [28], is to construct a norm $\|\cdot\|_{\operatorname{Det}\left(H^{\bullet}(M, E)\right)}^{\mathrm{RS}}$ on the determinant line of $H^{\bullet}(M, E)$, called the Ray-Singer metric, which is independent of $g^{M}$ and $h^{E}$. We now briefly recall this construction.

For each $\lambda \geq 0$, the cohomology of the finite dimensional complex $\left(\check{\Omega}_{[0, \lambda]}^{\bullet}(M, E), \nabla\right)$ is naturally isomorphic to $H^{\bullet}(M, E)$. Identifying these two cohomology spaces, we then obtain from (2-14) an isomorphism

$$
\phi_{\lambda}=\phi_{\check{\Omega}_{[0, \lambda]}^{\bullet}(M, E)}: \operatorname{Det}\left(\check{\Omega}_{[0, \lambda]}^{\bullet}(M, E)\right) \longrightarrow \operatorname{Det}\left(H^{\bullet}(M, E)\right) .
$$

The scalar product $\langle\cdot, \cdot\rangle$ on $\check{\Omega}_{[0, \lambda]}^{\bullet}(M, E) \subset \Omega^{\bullet}(M, E)$ defined by $g^{M}$ and $h^{E}$ induces a metric on the determinant line $\operatorname{Det}\left(\check{\Omega}_{[0, \lambda]}^{\bullet}(M, E)\right)$. Let $\|\cdot\|_{\lambda}$ denote the metric on $\operatorname{Det}\left(H^{\bullet}(M, E)\right)$ such that the isomorphism (11-5) is an isometry. It is well known, cf, for example, [4, Theorem 1.1], that for $0 \leq \lambda \leq \mu$

$$
\|\cdot\|_{\mu}=\|\cdot\|_{\lambda} \cdot T_{(\lambda, \mu]}^{\mathrm{RS}} \cdot
$$


The Ray-Singer metric on $\operatorname{Det}\left(H^{\bullet}(M, E)\right)$ is defined by the formula

$$
\|\cdot\|_{\operatorname{Det}(H \bullet(M, E))}^{\mathrm{RS}}:=\|\cdot\|_{\lambda} \cdot T_{(\lambda, \infty)}^{\mathrm{RS}}, \quad \lambda \geq 0 .
$$

It follows immediately from (11-4) and (11-6) that $\|\cdot\|_{\operatorname{Det}(H \bullet(M, E))}^{\mathrm{RS}}$ is independent of the choice of $\lambda \geq 0$.

Theorem 11.3 Let $E$ be a complex vector bundle over a closed, oriented, odddimensional manifold $M$ and let $\nabla$ be a flat connection on $E$. Then

$$
\left\|\rho_{\mathrm{an}}\right\|_{\operatorname{Det}\left(H^{\bullet}(M, E)\right)}^{\mathrm{RS}}=e^{\pi \operatorname{Im} \eta\left(\nabla, g^{M}\right)},
$$

where

$$
\eta\left(\nabla, g^{M}\right)=\eta\left(\mathcal{B}_{\text {even }}\left(\nabla, g^{M}\right)\right) .
$$

In particular, if $\nabla$ is a Hermitian connection, then $\eta\left(\nabla, g^{M}\right) \in \mathbb{R}$ and

$$
\left\|\rho_{\mathrm{an}}\right\|_{\operatorname{Det}\left(H^{\bullet}(M, E)\right)}^{\mathrm{RS}}=1 .
$$

The rest of this section is concerned with the proof of Theorem 11.3.

\subsection{Choice of the spectral cut}

The determinants in (11-2) and (11-3) are defined using the spectral cut $R_{-\pi}$ along the negative real axis. Since the spectrum of the operator $\Delta_{k}$ lies on the positive real axis, we can replace $R_{-\pi}$ with a spectral cut $R_{\gamma}$ for any nonzero $-\pi \leq \gamma<\pi$ without changing the value of $\operatorname{Det}_{\theta}^{\prime}\left(\Delta_{k}\right)$. In particular, we can take the spectral cut along $R_{2 \theta}$, where $\theta \in(-\pi / 2,0)$ is any Agmon angle for the odd signature operator $\mathcal{B}=\mathcal{B}\left(\nabla, g^{M}\right)$ such that there are no eigenvalues of $\mathcal{B}$ in the solid angles $L_{[-\theta-\pi,-\pi / 2]}, L_{(-\pi / 2, \theta]}$, $L_{[-\theta, \pi / 2)}$, and $L_{(\pi / 2, \theta+\pi]}$. We fix such an angle $\theta$ till the end of this section.

\subsection{The Ray-Singer metric and the dual connection}

Let $\nabla^{\prime}$ be the connection dual to $\nabla$ with respect to the Hermitian metric $h^{E}$, cf Section 10.1 , and let $E^{\prime}$ denote the flat bundle $\left(E, \nabla^{\prime}\right)$. Let

$$
\Delta^{\prime}=\left(\nabla^{\prime}\right)^{*} \nabla^{\prime}+\nabla^{\prime}\left(\nabla^{\prime}\right)^{*}
$$

denote the Laplacian of the connection $\nabla^{\prime}$. For $\lambda \geq 0$, we denote by

$$
\check{\Omega}_{[0, \lambda]}^{\bullet}\left(M, E^{\prime}\right) \subset \Omega^{\bullet}(M, E)
$$

the image of the spectral projection $\Pi_{\Delta^{\prime},[0, \lambda]}$, cf Section 6.10. As in Section 11.2, we use the scalar product induced by $g^{M}$ and $h^{E}$ on $\check{\Omega}_{[0, \lambda]}^{\bullet}\left(M, E^{\prime}\right)$ to construct a metric 
$\|\cdot\|_{\lambda}^{\prime}$ on $\operatorname{Det}\left(H^{\bullet}\left(M, E^{\prime}\right)\right)$ and we define the Ray-Singer metric on $\operatorname{Det}\left(H^{\bullet}\left(M, E^{\prime}\right)\right)$ by the formula

$$
\|\cdot\|_{\operatorname{Det}\left(H^{\bullet}\left(M, E^{\prime}\right)\right)}^{\mathrm{RS}}:=\|\cdot\|_{\lambda}^{\prime} \cdot T_{(\lambda, \infty)}^{\mathrm{RS}}\left(\nabla^{\prime}\right) .
$$

\subsection{Comparison between the Ray-Singer metrics associated to a connec- tion and to its dual}

From (10-8) we conclude that

$$
\Delta^{\prime}=\Gamma \circ \Delta \circ \Gamma
$$

Hence, for each $\lambda \geq 0, j=0, \ldots, d$,

$$
\Gamma\left(\check{\Omega}_{[0, \lambda]}^{j}\left(M, E^{\prime}\right)\right)=\check{\Omega}_{[0, \lambda]}^{d-j}(M, E) .
$$

Recall that the notation $h^{E}(\alpha \wedge \beta)$ was introduced in (10-1). It follows from (11-11) and (10-1) that the map

$$
\left(\omega^{\prime}, \omega\right) \mapsto \int_{M} h^{E}\left(\Gamma \omega^{\prime} \wedge \omega\right), \quad \omega \in \check{\Omega}_{[0, \lambda]}^{\bullet}(M, E), \omega^{\prime} \in \check{\Omega}_{[0, \lambda]}^{\bullet}\left(M, E^{\prime}\right)
$$

defines a non-degenerate sesquilinear pairing between $\check{\Omega}_{[0, \lambda]}^{\bullet}\left(M, E^{\prime}\right)$ and $\check{\Omega}_{[0, \lambda]}^{\bullet}(M, E)$ and, hence, identifies $\check{\Omega}_{[0, \lambda]}^{\bullet}\left(M, E^{\prime}\right)$ with the dual space of $\check{\Omega}_{[0, \lambda]}^{\bullet}(M, E)$. Moreover, this identification preserves the scalar products induced by $g^{M}$ and $h^{E}$ on $\check{\Omega}_{[0, \lambda]}^{\bullet}\left(M, E^{\prime}\right)$ and on the dual to $\check{\Omega}_{[0, \lambda]}^{\bullet}(M, E)$. Hence, the anti-linear isomorphism $(10-4)$ is an isometry with respect to the metrics $\|\cdot\|_{\lambda}$ and $\|\cdot\|_{\lambda}^{\prime}$. In particular,

$$
\left\|\rho_{\mathrm{an}}(\nabla)\right\|_{\lambda}=\left\|\alpha\left(\rho_{\mathrm{an}}(\nabla)\right)\right\|_{\lambda}^{\prime} .
$$

It follows now from (10-6) that

$$
\left\|\rho_{\mathrm{an}}(\nabla)\right\|_{\lambda}=\left\|\rho_{\mathrm{an}}\left(\nabla^{\prime}\right)\right\|_{\lambda}^{\prime} \cdot e^{2 \pi \operatorname{Im} \eta\left(\nabla, g^{M}\right)} .
$$

A verbatim repetition of the proof of [9, Lemma 8.8] yields that for each $\lambda \geq 0$

$$
T_{(\lambda, \infty)}^{\mathrm{RS}}\left(\nabla^{\prime}\right)=T_{(\lambda, \infty)}^{\mathrm{RS}}(\nabla) .
$$

Hence, from (11-7), (11-10), and (11-13), we conclude that

$$
\left\|\rho_{\mathrm{an}}(\nabla)\right\|_{\operatorname{Det}\left(H^{\bullet}(M, E)\right)}^{\mathrm{RS}}=\left\|\rho_{\mathrm{an}}\left(\nabla^{\prime}\right)\right\|_{\operatorname{Det}\left(H^{\bullet}\left(M, E^{\prime}\right)\right)}^{\mathrm{RS}} \cdot e^{2 \pi \operatorname{Im} \eta\left(\nabla, g^{M}\right)} .
$$




\subsection{Direct sum of a connection and its dual}

Let

$$
\tilde{\nabla}=\left(\begin{array}{cc}
\nabla & 0 \\
0 & \nabla^{\prime}
\end{array}\right)
$$

denote the flat connection on $E \oplus E$ obtained as a direct sum of the connections $\nabla$ and $\nabla^{\prime}$. Clearly, for each $\lambda \geq 0$,

$$
\begin{aligned}
\xi_{\lambda}\left(\tilde{\nabla}, g^{M}, \theta\right) & =\xi_{\lambda}\left(\nabla, g^{M}, \theta\right)+\xi_{\lambda}\left(\nabla^{\prime}, g^{M}, \theta\right), \\
\eta_{\lambda}\left(\tilde{\nabla}, g^{M}\right) & =\eta_{\lambda}\left(\nabla, g^{M}\right)+\eta_{\lambda}\left(\nabla^{\prime}, g^{M}\right) .
\end{aligned}
$$

From Lemma 4.7 we obtain for $\lambda \geq 0$

$$
\text { (11-17) } \rho_{\Gamma_{[0, \lambda]}}\left(\widetilde{\nabla}, g^{M}\right)=\mu_{H} \bullet(M, E), H^{\bullet}\left(M, E^{\prime}\right)\left(\rho_{\Gamma_{[0, \lambda]}}\left(\nabla, g^{M}\right) \otimes \rho_{\Gamma_{[0, \lambda]}}\left(\nabla^{\prime}, g^{M}\right)\right) \text {. }
$$

Hence, in view of (7-15), (8-5), and Definition 9.8, we obtain

$$
\rho_{\mathrm{an}}(\tilde{\nabla})=\mu_{H^{\bullet}(M, E), H^{\bullet}\left(M, E^{\prime}\right)}\left(\rho_{\mathrm{an}}(\nabla) \otimes \rho_{\mathrm{an}}\left(\nabla^{\prime}\right)\right) .
$$

Let $\tilde{\Delta}=\widetilde{\nabla}^{*} \widetilde{\nabla}+\widetilde{\nabla} \widetilde{\nabla}^{*}$ be the Laplacian of the connection $\widetilde{\nabla}$ and let $\check{\Omega}_{[0, \lambda]}^{\bullet}\left(M, E \oplus E^{\prime}\right)$ denote the span of the eigenvectors of $\widetilde{\Delta}$ corresponding to eigenvalues which are $\leq \lambda$. As in Section 11.2, we use the scalar product induced by $g^{M}$ and $h^{E}$ on $\check{\Omega}_{[0, \lambda]}^{\bullet}\left(M, E \oplus E^{\prime}\right)$ to construct a metric $\|\cdot\|_{\lambda}^{\sim}$ on $\operatorname{Det}\left(H^{\bullet}\left(M, E \oplus E^{\prime}\right)\right)$ and we define the Ray-Singer metric on $\operatorname{Det}\left(H^{\bullet}\left(M, E \oplus E^{\prime}\right)\right)$ by the formula

$$
\|\cdot\|_{\operatorname{Det}\left(H^{\bullet}\left(M, E \oplus E^{\prime}\right)\right)}^{\mathrm{RS}}:=\|\cdot\|_{\lambda}^{\sim} \cdot T_{(\lambda, \infty)}^{\mathrm{RS}}(\tilde{\nabla}) .
$$

Since

$$
\check{\Omega}_{[0, \lambda]}^{\bullet}\left(M, E \oplus E^{\prime}\right)=\check{\Omega}_{[0, \lambda]}^{\bullet}(M, E) \oplus \check{\Omega}_{[0, \lambda]}^{\bullet}\left(M, E^{\prime}\right),
$$

it follows from the definition (2-5) of the fusion isomorphism that, for any $h \in$ $H^{\bullet}(M, E)$ and $h^{\prime} \in H^{\bullet}\left(M, E^{\prime}\right)$,

$$
\left\|\mu_{H^{\bullet}(M, E), H^{\bullet}\left(M, E^{\prime}\right)}\left(h \otimes h^{\prime}\right)\right\|_{\lambda}^{\sim}=\|h\|_{\lambda} \cdot\left\|h^{\prime}\right\|_{\lambda}^{\prime} .
$$

Therefore, we obtain from (11-18)

$$
\left\|\rho_{\mathrm{an}}(\tilde{\nabla})\right\|_{\lambda}^{\tilde{2}}=\left\|\rho_{\mathrm{an}}(\nabla)\right\|_{\lambda} \cdot\left\|\rho_{\mathrm{an}}\left(\nabla^{\prime}\right)\right\|_{\lambda}^{\prime} .
$$

Since

$$
T_{(\lambda, \infty)}^{\mathrm{RS}}(\tilde{\nabla})=T_{(\lambda, \infty)}^{\mathrm{RS}}(\nabla) \cdot T_{(\lambda, \infty)}^{\mathrm{RS}}\left(\nabla^{\prime}\right),
$$

we conclude from (11-19) and (11-21) that $(11-22)$

$$
\left\|\rho_{\mathrm{an}}(\widetilde{\nabla})\right\|_{\operatorname{Det}\left(H^{\bullet}\left(M, E \oplus E^{\prime}\right)\right)}^{\mathrm{RS}}=\left\|\rho_{\mathrm{an}}(\nabla)\right\|_{\operatorname{Det}\left(H^{\bullet}(M, E)\right)}^{\mathrm{RS}} \cdot\left\|\rho_{\mathrm{an}}\left(\nabla^{\prime}\right)\right\|_{\operatorname{Det}\left(H^{\bullet}\left(M, E^{\prime}\right)\right)}^{\mathrm{RS}} .
$$


Combining the later equality with (11-15), we get

(11-23) $\left\|\rho_{\mathrm{an}}(\tilde{\nabla})\right\|_{\operatorname{Det}\left(H^{\bullet}\left(M, E \oplus E^{\prime}\right)\right)}^{\mathrm{RS}}=\left(\left\|\rho_{\mathrm{an}}(\nabla)\right\|_{\operatorname{Det}\left(H^{\bullet}(M, E)\right)}^{\mathrm{RS}}\right)^{2} \cdot e^{-2 \pi \operatorname{Im} \eta\left(\nabla, g^{M}\right)}$.

Hence, (11-8) is equivalent to the equality

$$
\left\|\rho_{\mathrm{an}}(\widetilde{\nabla})\right\|_{\operatorname{Det}\left(H^{\bullet}\left(M, E \oplus E^{\prime}\right)\right)}^{\mathrm{RS}}=1 .
$$

\subsection{Deformation of the chirality operator}

We will prove (11-24) by a deformation argument. For $t \in[-\pi / 2, \pi / 2]$ introduce the rotation $U_{t}$ on

$$
\Omega^{\bullet}:=\Omega^{\bullet}(M, E) \oplus \Omega^{\bullet}(M, E)
$$

given by

$$
U_{t}=\left(\begin{array}{cc}
\cos t & -\sin t \\
\sin t & \cos t
\end{array}\right)
$$

Note that $U_{t}^{-1}=U_{-t}$. Denote by $\widetilde{\Gamma}(t)$ the deformation of the chirality operator, defined by

$$
\widetilde{\Gamma}(t)=U_{t} \circ\left(\begin{array}{cc}
\Gamma & 0 \\
0 & -\Gamma
\end{array}\right) \circ U_{t}^{-1}=\Gamma \circ\left(\begin{array}{cc}
\cos 2 t & \sin 2 t \\
\sin 2 t & -\cos 2 t
\end{array}\right) .
$$

Then

$$
\widetilde{\Gamma}(0)=\left(\begin{array}{cc}
\Gamma & 0 \\
0 & -\Gamma
\end{array}\right), \quad \widetilde{\Gamma}(\pi / 4)=\left(\begin{array}{cc}
0 & \Gamma \\
\Gamma & 0
\end{array}\right)
$$

\subsection{Deformation of the odd signature operator}

Consider a one-parameter family of operators $\tilde{\mathcal{B}}(t): \Omega^{\bullet} \rightarrow \Omega^{\bullet}(t \in[-\pi / 2, \pi / 2])$ defined by the formula

$$
\widetilde{\mathcal{B}}(t):=\widetilde{\Gamma}(t) \tilde{\nabla}+\tilde{\nabla} \widetilde{\Gamma}(t) .
$$

Then

$$
\widetilde{\mathcal{B}}(0)=\left(\begin{array}{cc}
\mathcal{B} & 0 \\
0 & -\mathcal{B}^{\prime}
\end{array}\right)
$$

and

$$
\widetilde{\mathcal{B}}(\pi / 4)=\left(\begin{array}{cc}
0 & \Gamma \nabla^{\prime}+\nabla \Gamma \\
\Gamma \nabla+\nabla^{\prime} \Gamma & 0
\end{array}\right) .
$$


Hence, using (10-8), we obtain

$$
\widetilde{\mathcal{B}}(\pi / 4)^{2}=\left(\begin{array}{cc}
\Delta & 0 \\
0 & \Delta^{\prime}
\end{array}\right)=\tilde{\Delta}
$$

Set

$$
\begin{aligned}
\Omega_{+}^{\bullet}(t) & :=\operatorname{Ker} \tilde{\nabla} \tilde{\Gamma}(t) \\
\Omega_{-}^{\bullet} & :=\operatorname{Ker} \tilde{\nabla}=\operatorname{Ker} \nabla \oplus \operatorname{Ker} \nabla^{\prime} .
\end{aligned}
$$

Note that $\Omega_{-}^{\bullet}$ is independent of $t$. Since the operators $\tilde{\nabla}$ and $\widetilde{\Gamma}(t)$ commute with $\widetilde{\mathcal{B}}(t)$, the spaces $\Omega_{+}^{\bullet}(t)$ and $\Omega_{-}^{\bullet}$ are invariant for $\tilde{\mathcal{B}}(t)$.

Let $\mathcal{I}$ be an interval of the form $[0, \lambda],(\lambda, \mu]$, or $(\lambda, \infty](\mu \geq \lambda \geq 0)$. Denote

$$
\Omega_{\mathcal{I}}^{\bullet}(t):=\Pi_{\widetilde{\mathcal{B}}(t)^{2}, \mathcal{I}}\left(\Omega^{\bullet}(t)\right) \subset \Omega^{\bullet}(t),
$$

where $\Pi_{\widetilde{\mathcal{B}}(t)^{2}, \mathcal{I}}$ is the spectral projection of $\widetilde{\mathcal{B}}(t)^{2}$ corresponding to $\mathcal{I}$, cf Section 6.10. For $j=0, \ldots, d$, set $\Omega_{\mathcal{I}}^{j}(t)=\Omega_{\mathcal{I}}^{\bullet}(t) \cap \Omega^{j}$ and

$$
\Omega_{ \pm, \mathcal{I}}^{j}(t):=\Omega_{ \pm}^{j}(t) \cap \Omega_{\mathcal{I}}^{j}(t) .
$$

For each $\lambda \geq 0, t \in(-\pi / 2, \pi / 2)$, the space $\Omega_{(\lambda, \infty)}^{\bullet}(t)$ is invariant by $\widetilde{\mathcal{B}}^{(\lambda, \infty)}(t)$ and the operator $\tilde{\mathcal{B}}^{(\lambda, \infty)}(t): \Omega_{(\lambda, \infty)}^{\bullet}(t) \rightarrow \Omega_{(\lambda, \infty)}^{\bullet}(t)$ is bijective. Since the range of the restriction of $\widetilde{\Gamma}(t) \widetilde{\nabla}$ to $\Omega_{(\lambda, \infty)}^{\bullet}(t)$ is contained in $\Omega_{+,(\lambda, \infty)}^{\bullet}(t)$ whereas the range of the restriction of $\widetilde{\nabla} \widetilde{\Gamma}(t)$ to $\Omega_{(\lambda, \infty)}^{\bullet}(t)$ is contained in $\Omega_{-,(\lambda, \infty)}^{\bullet}(t)$, it follows from the surjectivity of $\widetilde{\mathcal{B}}^{(\lambda, \infty)}(t)$ that

$$
\Omega_{+,(\lambda, \infty)}^{\bullet}(t)+\Omega_{-,(\lambda, \infty)}^{\bullet}(t)=\Omega_{(\lambda, \infty)}^{\bullet}(t), \quad t \in[-\pi / 2, \pi / 2]
$$

Since the kernel of the restriction of $\tilde{\nabla} \widetilde{\Gamma}(t)$ to $\Omega_{(\lambda, \infty)}^{\bullet}(t)$ is equal to $\Omega_{+,(\lambda, \infty)}^{\bullet}(t)$ whereas the kernel of the restriction of $\widetilde{\Gamma}(t) \tilde{\nabla}$ to $\Omega_{(\lambda, \infty)}^{\bullet}(t)$ is equal to $\Omega_{-,(\lambda, \infty)}^{\bullet}(t)$, it follows from the injectivity of $\widetilde{\mathcal{B}}^{(\lambda, \infty)}(t)$ that

$$
\Omega_{+,(\lambda, \infty)}^{\bullet}(t) \cap \Omega_{-,(\lambda, \infty)}^{\bullet}(t)=\{0\}, \quad t \in[-\pi / 2, \pi / 2] .
$$

Combining (11-32) and (11-33) we obtain

$$
\Omega_{(\lambda, \infty)}^{\bullet}(t)=\Omega_{+,(\lambda, \infty)}^{\bullet}(t) \oplus \Omega_{-,(\lambda, \infty)}^{\bullet}(t), \quad t \in[-\pi / 2, \pi / 2]
$$

We define $\tilde{\mathcal{B}}_{j}^{\mathcal{I}}(t), \widetilde{\mathcal{B}}_{\text {even }}^{\mathcal{I}}(t), \widetilde{\mathcal{B}}_{\text {odd }}^{\mathcal{I}}(t), \widetilde{\mathcal{B}}_{j}^{ \pm, \mathcal{I}}(t), \widetilde{\mathcal{B}}_{\text {even }}^{ \pm, \mathcal{I}}(t), \widetilde{\mathcal{B}}_{\text {odd }}^{ \pm, \mathcal{I}}(t)$, etc. in the same way as the corresponding maps were defined in Section 7.6. 


\subsection{The graded determinant of the deformed odd signature operator}

By Definition 5.5 and Definition 6.13, for every $\mathcal{I}$ of the form $[0, \lambda],(\lambda, \mu]$, or $(\lambda, \infty]$ $(\mu \geq \lambda \geq 0)$, the graded determinant of $\widetilde{\mathcal{B}}_{\text {even }}^{\mathcal{I}}(t)$ is given by the formula

$$
\operatorname{Det}_{\mathrm{gr}, \theta}^{\prime}\left(\tilde{\mathcal{B}}_{\text {even }}^{\mathcal{I}}(t)\right):=e^{\operatorname{LDet}_{\mathrm{gr}, \theta}^{\prime}\left(\tilde{\mathcal{B}}_{\text {even }}^{\mathcal{I}}(t)\right)},
$$

where $\theta$ is an Agmon angle for $\tilde{\mathcal{B}}_{\text {even }}^{\mathcal{I}}(t)$ and

$$
\operatorname{LDet}_{\mathrm{gr}, \theta}^{\prime}\left(\widetilde{\mathcal{B}}_{\text {even }}^{\mathcal{I}}(t)\right):=\operatorname{LDet}_{\theta}^{\prime}\left(\widetilde{\mathcal{B}}_{\text {even }}^{+, \mathcal{I}}(t)\right)-\operatorname{LDet}_{\theta}^{\prime}\left(-\widetilde{\mathcal{B}}_{\text {even }}^{-, \mathcal{I}}(t)\right) \in \mathbb{C} .
$$

Since $\widetilde{\Gamma}(t)$ commutes with $\widetilde{\mathcal{B}}(t)$, we easily obtain

$$
\widetilde{\mathcal{B}}_{j}^{+, \mathcal{I}}(t)=\widetilde{\Gamma}(t) \circ \widetilde{\mathcal{B}}_{d-j}^{-, \mathcal{I}}(t) \circ \widetilde{\Gamma}(t), \quad j=0, \ldots, d .
$$

Therefore, (11-36) can be rewritten as

$$
\operatorname{LDet}_{\mathrm{gr}, \theta}^{\prime}\left(\widetilde{\mathcal{B}}_{\mathrm{even}}^{\mathcal{I}}(t)\right):=\sum_{j=0}^{d}(-1)^{j} \operatorname{LDet}_{\theta}^{\prime}\left((-1)^{j} \widetilde{\mathcal{B}}_{j}^{+, \mathcal{I}}(t)\right) .
$$

Lemma 11.11 Suppose that $\theta \in(-\pi / 2,0)$ is an Agmon angle for the operator $\widetilde{\mathcal{B}}_{\text {even }}^{(\lambda, \infty)}(\pi / 4)$. Then, for each $\lambda \geq 0$,

$$
\left|\operatorname{Det}_{\mathrm{gr}, \theta}\left(\widetilde{\mathcal{B}}_{\text {even }}^{(\lambda, \infty)}(\pi / 4)\right)\right|=\frac{1}{T_{(\lambda, \infty)}^{\mathrm{RS}}(\widetilde{\nabla})} .
$$

Proof It follows from (11-29) that the operator $\widetilde{\mathcal{B}}_{\text {even }}^{(\lambda, \infty)}(\pi / 4)$ is self-adjoint. Hence, $\eta\left(0, \widetilde{\mathcal{B}}_{\text {even }}^{(\lambda, \infty)}(\pi / 4)\right)$ and $\zeta_{2 \theta}\left(0, \widetilde{\mathcal{B}}_{\text {even }}^{(\lambda, \infty)}(\pi / 4)^{2}\right)$ are real, cf, for example, [9, Theorem A.2]. Thus, from (11-30), (11-37), and (6-38), we conclude that

$$
\begin{aligned}
\operatorname{Re}_{\operatorname{LDet}}, \theta\left(\widetilde{\mathcal{B}}_{\mathrm{even}}^{(\lambda, \infty)}(\pi / 4)\right) & =\frac{1}{2} \sum_{j=0}^{d}(-1)^{j} \operatorname{LDet}_{2 \theta}\left[\left.\widetilde{\Delta}^{(\lambda, \infty)}\right|_{\Omega_{+,(\lambda, \infty)}^{j}(\pi / 4)}\right] \\
& =\frac{1}{2} \sum_{j=0}^{d}(-1)^{j} \operatorname{LDet}_{-\pi}\left[\left.\widetilde{\Delta}_{j}^{(\lambda, \infty)}\right|_{\Omega_{+,(\lambda, \infty)}^{j}(\pi / 4)}\right] .
\end{aligned}
$$

As on [11, page 340] (see also [9, Section 8.4]), one shows that the right hand side of (11-39) is equal to

$$
\frac{1}{2} \sum_{j=0}^{d}(-1)^{j+1} j \text { LDet }_{-\pi}\left[\widetilde{\Delta}_{j}^{(\lambda, \infty)}\right] .
$$

Hence, equality (11-38) follows from(11-3) and (11-35). 


\subsection{Deformation of the canonical element of the determinant line}

Since the operators $\widetilde{\nabla}$ and $\widetilde{\mathcal{B}}(t)^{2}$ commute, the space $\Omega_{\mathcal{I}}^{\bullet}(t)$ is invariant under $\widetilde{\nabla}$, ie, it is a subcomplex of $\Omega^{\bullet}$. The same arguments as in the proof of Lemma 5.9 show that, for every $\lambda \geq 0$, the complex $\Omega_{(\lambda, \infty)}^{\bullet}(t)$ is acyclic and, hence, the cohomology of the finite dimensional complex $\Omega_{[0, \lambda]}^{\bullet}(t)$ is naturally isomorphic to

$$
H^{\bullet}\left(M, E \oplus E^{\prime}\right) \simeq H^{\bullet}(M, E) \oplus H^{\bullet}\left(M, E^{\prime}\right)
$$

Let $\widetilde{\Gamma}_{[0, \lambda]}(t)$ denote the restriction of $\widetilde{\Gamma}(t)$ to $\Omega_{[0, \lambda]}^{\bullet}(t)$. As $\widetilde{\Gamma}(t)$ and $\widetilde{\mathcal{B}}(t)^{2}$ commute, it follows that $\widetilde{\Gamma}_{[0, \lambda]}(t)$ maps $\Omega_{[0, \lambda]}^{\bullet}(t)$ onto itself and, therefore, is a chirality operator for $\Omega_{[0, \lambda]}^{\bullet}(t)$. Let

$$
\rho_{\widetilde{\Gamma}_{[0, \lambda]}^{(t)}}(t) \in \operatorname{Det}\left(H^{\bullet}\left(M, E \oplus E^{\prime}\right)\right)
$$

denote the refined torsion of the finite dimensional complex $\left(\Omega_{[0, \lambda]}^{\bullet}(t), \widetilde{\nabla}\right)$ corresponding to the chirality operator $\widetilde{\Gamma}_{[0, \lambda]}(t)$, cf Definition 4.3 .

For each $t \in(-\pi / 2, \pi / 2)$ fix an Agmon angle $\theta=\theta(t) \in(-\pi / 2,0)$ for $\widetilde{\mathcal{B}}_{\text {even }}(t)$ and define the element $\rho(t) \in \operatorname{Det}\left(H^{\bullet}\left(M, E \oplus E^{\prime}\right)\right)$ by the formula

$$
\rho(t):=\operatorname{Det}_{\mathrm{gr}, \theta}\left(\widetilde{\mathcal{B}}_{\text {even }}^{(\lambda, \infty)}(t)\right) \cdot \rho_{\widetilde{\Gamma}_{[0, \lambda]}(t)}(t),
$$

where $\lambda$ is any non-negative real number. It follows from Proposition 5.10 that $\rho(t)$ is independent of the choice of $\lambda \geq 0$.

\subsection{The Ray-Singer norm of $\rho(t)$}

For $t \in[-\pi / 2, \pi / 2], \lambda \geq 0$, set

$$
\begin{aligned}
\xi_{\lambda}(t, \theta) & :=\frac{1}{2} \sum_{j=0}^{d}(-1)^{j+1} j \operatorname{LDet}_{2 \theta}\left[\left.\widetilde{\mathcal{B}}_{\text {even }}^{(\lambda, \infty)}(t)^{2}\right|_{\Omega_{(\lambda, \infty)}^{j}(t)}\right], \\
\eta_{\lambda}(t) & :=\eta\left(\widetilde{\mathcal{B}}_{\text {even }}^{(\lambda, \infty)}(t)\right) .
\end{aligned}
$$

From (6-16), we see that $\operatorname{Re} \xi_{\lambda}(t, \theta)$ is independent of the choice of the angle $\theta \in$ $(-\pi / 2,0)$ such that both $\theta$ and $\theta+\pi$ are Agmon angles for $\widetilde{\mathcal{B}}(t)$. Hence, for any such angle $\theta \in(-\pi / 2,0)$, we obtain from (11-41) and (8-5),

$$
\|\rho(t)\|_{\operatorname{Det}\left(H^{\bullet}\left(M, E \oplus E^{\prime}\right)\right)}^{\mathrm{RS}}=\left\|\rho_{\widetilde{\Gamma}_{[0, \lambda]}(t)}(t) \cdot e^{\xi_{\lambda}(t, \theta)}\right\|_{\operatorname{Det}\left(H^{\bullet}\left(M, E \oplus E^{\prime}\right)\right)}^{\mathrm{RS}} \cdot e^{\pi \operatorname{Im} \eta_{\lambda}(t)} .
$$


Since the rank of the operator $\widetilde{\mathcal{B}}_{\text {even }}^{[0, \lambda]}(t)$ is finite, $\eta\left(\widetilde{\mathcal{B}}_{\text {even }}^{[0, \lambda]}(t)\right) \in \frac{1}{2} \mathbb{Z}$. Hence,

$$
\operatorname{Im} \eta_{\lambda}(t)=\operatorname{Im}\left(\eta\left(\widetilde{\mathcal{B}}_{\text {even }}(t)\right)-\eta\left(\widetilde{\mathcal{B}}_{\text {even }}^{[0, \lambda]}(t)\right)\right)=\operatorname{Im} \eta\left(\widetilde{\mathcal{B}}_{\text {even }}(t)\right)
$$

is independent of $\lambda \geq 0$. We conclude now from (11-44) that

$$
\left\|\rho_{\widetilde{\Gamma}_{[0, \lambda]}(t)}(t) \cdot e^{\xi_{\lambda}(t, \theta)}\right\|_{\operatorname{Det}\left(H \bullet\left(M, E \oplus E^{\prime}\right)\right)}^{\mathrm{RS}}
$$

is independent of $\lambda \geq 0$.

\subsection{The Ray-Singer norm of $\rho(0)$}

By (11-28),

$$
\eta_{\lambda}(0)=\eta\left(\mathcal{B}_{\text {even }}^{(\lambda, \infty)}\right)+\eta\left(-\mathcal{B}_{\text {even }}^{\prime(\lambda, \infty)}\right) .
$$

Since the operator $\mathcal{B}_{\text {even }}^{(\lambda, \infty)}$ is invertible $m_{0}\left(\mathcal{B}_{\text {even }}^{(\lambda, \infty)}\right)=0$ (see Section 6.15 for the definition of $m_{0}$ ). It then follows from the definition (6-35) of the $\eta$-invariant that

$$
\eta\left(-\mathcal{B}_{\text {even }}^{\prime(\lambda, \infty)}\right)=-\eta\left(\mathcal{B}_{\text {even }}^{\prime(\lambda, \infty)}\right) .
$$

Hence, using definition (8-1) of $\eta_{\lambda}\left(\nabla, g^{M}\right)$ and formula (10-11) for $\eta_{\lambda}\left(\nabla^{\prime}, g^{M}\right)$, we get

$$
\eta_{\lambda}(0)=\eta_{\lambda}\left(\nabla, g^{M}\right)-\eta_{\lambda}\left(\nabla^{\prime}, g^{M}\right)=2 i \operatorname{Im} \eta_{\lambda}\left(\nabla, g^{M}\right) .
$$

Using again the invertibility of $\mathcal{B}_{\text {even }}^{(\lambda, \infty)}$, we obtain from (6-38) and (11-45) (and (8-1) and (10-11)), that

$$
\begin{aligned}
\operatorname{Det}_{\mathrm{gr}, \theta}\left(-\mathcal{B}_{\mathrm{even}}^{\prime(\lambda, \infty)}\right) & =\operatorname{Det}_{\mathrm{gr}, \theta}\left(\mathcal{B}_{\mathrm{even}}^{(\lambda, \infty)}\right) \cdot e^{2 \pi i \eta\left(\mathcal{B}_{\mathrm{even}}^{\prime(\lambda, \infty)}\right)} \\
& =\operatorname{Det}_{\mathrm{gr}, \theta}\left(\mathcal{B}_{\mathrm{even}}^{\prime(\lambda, \infty)}\right) \cdot e^{2 \pi i \bar{\eta}\left(\nabla, g^{M}\right)}
\end{aligned}
$$

Hence, from (11-28), we get

$$
\text { (11-47) } \operatorname{Det}_{\mathrm{gr}, \theta}\left(\widetilde{\mathcal{B}}_{\text {even }}^{(\lambda, \infty)}(0)\right)=\operatorname{Det}_{\mathrm{gr}, \theta}\left(\mathcal{B}_{\text {even }}^{(\lambda, \infty)}\right) \cdot \operatorname{Det}_{\mathrm{gr}, \theta}\left(\mathcal{B}_{\text {even }}^{(\lambda, \infty)}\right) \cdot e^{2 \pi i \bar{\eta}_{\lambda}\left(\nabla, g^{M}\right)} \text {. }
$$

From (11-28) we conclude that $\Omega_{[0, \lambda]}^{\bullet}(0)=\Omega_{[0, \lambda]}^{\bullet}(M, E) \oplus \Omega_{[0, \lambda]}^{\bullet}\left(M, E^{\prime}\right)$. It follows now from (11-26) and (4-6) that

$$
\rho_{\widetilde{\Gamma}_{[0, \lambda]}(0)}(0)=\mu_{H^{\bullet}(M, E), H^{\bullet}\left(M, E^{\prime}\right)}\left(\rho_{\Gamma_{[0, \lambda]}}\left(\nabla, g^{M}\right) \otimes \rho_{-\Gamma_{[0, \lambda]}}\left(\nabla^{\prime}, g^{M}\right)\right) .
$$

From (4-1) and definition (4-3) of the element $\rho$, we conclude that

$$
\rho_{-\Gamma_{[0, \lambda]}}\left(\nabla^{\prime}, g^{M}\right)=(-1)^{\sum_{j=0}^{r-1} \operatorname{dim} \Omega_{[0, \lambda]}^{j}\left(M, E^{\prime}\right)} \cdot \rho_{\Gamma_{[0, \lambda]}}\left(\nabla^{\prime}, g^{M}\right) .
$$


Hence, by (11-20) and (11-48), we have

$$
\begin{aligned}
& (11-50) \quad\left\|\rho_{\widetilde{\Gamma}_{[0, \lambda]}(0)}(0)\right\|_{\operatorname{Det}\left(H^{\bullet}\left(M, E \oplus E^{\prime}\right)\right)}^{\mathrm{RS}} \\
& \quad=\left\|\rho_{\Gamma_{[0, \lambda]}}\left(\nabla, g^{M}\right)\right\|_{\operatorname{Det}\left(H^{\bullet}\left(M, E^{\prime}\right)\right)}^{\mathrm{RS}} \cdot\left\|\rho_{\Gamma_{[0, \lambda]}}\left(\nabla^{\prime}, g^{M}\right)\right\|_{\operatorname{Det}\left(H^{\bullet}\left(M, E^{\prime}\right)\right)}^{\mathrm{RS}}
\end{aligned}
$$

Combining (11-41), (11-47), and (11-50) with (7-15) and (9-14) (definition of the refined analytic torsion), we conclude that

$$
\begin{aligned}
& (11-51)\|\rho(0)\|_{\operatorname{Det}\left(H^{\bullet}\left(M, E \oplus E^{\prime}\right)\right)}^{\mathrm{RS}} \\
& \quad=\left\|\rho_{\mathrm{an}}(\nabla)\right\|_{\operatorname{Det}\left(H^{\bullet}\left(M, E^{\prime}\right)\right)}^{\mathrm{RS}} \cdot\left\|\rho_{\mathrm{an}}\left(\nabla^{\prime}\right)\right\|_{\operatorname{Det}\left(H^{\bullet}\left(M, E^{\prime}\right)\right)}^{\mathrm{RS}} \cdot e^{2 \pi \operatorname{Im} \eta_{\lambda}\left(\nabla, g^{M}\right)} .
\end{aligned}
$$

Hence, by (11-22),

$$
(11-52) \quad\|\rho(0)\|_{\operatorname{Det}\left(H^{\bullet}\left(M, E \oplus E^{\prime}\right)\right)}^{\mathrm{RS}}=\left\|\rho_{\mathrm{an}}(\widetilde{\nabla})\right\|_{\operatorname{Det}\left(H^{\bullet}\left(M, E \oplus E^{\prime}\right)\right)}^{\mathrm{RS}} \cdot e^{2 \pi \operatorname{Im} \eta_{\lambda}\left(\nabla, g^{M}\right)} \text {. }
$$

\subsection{The Ray-Singer norm of $\rho(\pi / 4)$}

Recall that the norm $\|\cdot\|_{\lambda}^{\sim}$ was defined in Section 11.7. From (11-30) and Lemma 4.5, we obtain

$$
\left\|\rho_{\widetilde{\Gamma}_{[0, \lambda]}(\pi / 4)}(\pi / 4)\right\|_{\lambda}^{\sim}=1 .
$$

Hence, from Lemma 11.11, (11-41), and (11-19), we obtain

$$
\|\rho(\pi / 4)\|_{\operatorname{Det}\left(H^{\bullet}\left(M, E \oplus E^{\prime}\right)\right)}^{\mathrm{RS}}=1 \text {. }
$$

Proof of Theorem 11.3 From (11-44), (11-46), and (11-52), we obtain

$$
\left\|\rho_{\widetilde{\Gamma}_{[0, \lambda]}(0)}(0) \cdot e^{\xi_{\lambda}(0, \theta)}\right\|_{\operatorname{Det}\left(H^{\bullet}\left(M, E \oplus E^{\prime}\right)\right)}^{\mathrm{RS}}=\left\|\rho_{\mathrm{an}}(\widetilde{\nabla})\right\|_{\operatorname{Det}\left(H^{\bullet}\left(M, E \oplus E^{\prime}\right)\right)}^{\mathrm{RS}} \cdot
$$

By (11-29), the operator $\widetilde{\mathcal{B}}_{\text {even }}^{(\lambda, \infty)}(\pi / 4)$ is self-adjoint. Hence, $\eta_{\lambda}(\pi / 4) \in \mathbb{R}$. Therefore, we get from (11-44) and (11-53) that

$$
\left\|\rho_{\widetilde{\Gamma}_{[0, \lambda]}(\pi / 4)}(\pi / 4) \cdot e^{\xi_{\lambda}(\pi / 4, \theta)}\right\|_{\operatorname{Det}\left(H^{\bullet}\left(M, E \oplus E^{\prime}\right)\right)}^{\mathrm{RS}}=1 .
$$

From (11-54) and (11-55) we conclude that in order to prove (11-24) (and, hence, (11-8)) it suffices to show that

$$
\left\|\rho_{\widetilde{\Gamma}_{[0, \lambda]}(t)}(t) \cdot e^{\xi_{\lambda}(t, \theta)}\right\|_{\operatorname{Det}\left(H^{\bullet}\left(M, E \oplus E^{\prime}\right)\right)}^{\mathrm{RS}}
$$

is independent of $t$.

Fix $t_{0} \in[-\pi / 2, \pi / 2]$ and let $\lambda \geq 0$ be such that the operator $\widetilde{\mathcal{B}}_{\text {even }}\left(t_{0}\right)^{2}$ has no eigenvalues with absolute value $\lambda$. Choose an angle $\theta \in(-\pi / 2,0)$ such that both 
$\theta$ and $\theta+\pi$ are Agmon angles for $\widetilde{\mathcal{B}}\left(t_{0}\right)$. Then there exists $\delta>0$ such that for all $t \in\left(t_{0}-\delta, t_{0}+\delta\right) \cap[-\pi / 2, \pi / 2]$, the operator $\widetilde{\mathcal{B}}_{\text {even }}(t)^{2}$ has no eigenvalues with absolute value $\lambda$ and both $\theta$ and $\theta+\pi$ are Agmon angles for $\widetilde{\mathcal{B}}(t)$.

A verbatim repetition of the proof of Lemma 9.2 shows that

$$
\frac{d}{d t} \rho_{\widetilde{\Gamma}_{[0, \lambda]}(t)}(t) \cdot e^{\xi_{\lambda}(t, \theta)}=0 .
$$

Hence, (11-56) is independent of $t$.

\section{References}

[1] M F Atiyah, V K Patodi, I M Singer, Spectral asymmetry and Riemannian geometry. I, Math. Proc. Cambridge Philos. Soc. 77 (1975) 43-69 MR0397797

[2] M F Atiyah, V K Patodi, I M Singer, Spectral asymmetry and Riemannian geometry. II, Math. Proc. Cambridge Philos. Soc. 78 (1975) 405-432 MR0397798

[3] N Berline, E Getzler, M Vergne, Heat kernels and Dirac operators, volume 298 of Grundlehren der Mathematischen Wissenschaften, Springer, Berlin (1992) MR1215720

[4] J-M Bismut, W Zhang, An extension of a theorem by Cheeger and Müller, Astérisque (1992) 235 MR1185803 With an appendix by François Laudenbach.

[5] M Braverman, T Kappeler, A refinement of the Ray-Singer torsion, C. R. Math. Acad. Sci. Paris 341 (2005) 497-502 MR2180817

[6] M Braverman, T Kappeler, Comparison of the refined analytic and the BurgheleaHaller torsions (2006) arXiv:math.DG/0606398

[7] M Braverman, T Kappeler, Refined analytic torsion as an element of the determinant line, IHES preprint M/05/49 (2006) arXiv:math.GT/0510532

[8] M Braverman, T Kappeler, Ray-Singer type theorem for the refined analytic torsion, J. Funct. Anal. 243 (2007) 232-256 arXiv: math. DG/0603638

[9] M Braverman, T Kappeler, Refined Analytic Torsion, J. Diff. Geom. (to appear) arXiv:math.DG/0505537

[10] J Brüning, M Lesch, On the $\eta$-invariant of certain nonlocal boundary value problems, Duke Math. J. 96 (1999) 425-468 MR1666570

[11] D Burghelea, L Friedlander, T Kappeler, Asymptotic expansion of the Witten deformation of the analytic torsion, J. Funct. Anal. 137 (1996) 320-363 MR1387514

[12] D Burghelea, S Haller, Torsion as a function of the space of representations (2005) arXiv:math.DG/0507587 
[13] D Burghelea, S Haller, Complex valued Ray-Singer torsion II (2006) arXiv: math.DG/0610875

[14] D Burghelea, S Haller, Euler structures, the variety of representations and the MilnorTuraev torsion, Geom. Topol. 10 (2006) 1185-1238 MR2255496

[15] P Deligne, Le déterminant de la cohomologie, from: "Current trends in arithmetical algebraic geometry (Arcata, Calif., 1985)", Contemp. Math. 67, Amer. Math. Soc., Providence, RI (1987) 93-177 MR902592

[16] M Farber, Absolute torsion and eta-invariant, Math. Z. 234 (2000) 339-349 MR1765885

[17] M Farber, V Turaev, Absolute torsion, from: "Tel Aviv Topology Conference: Rothenberg Festschrift (1998)”, Contemp. Math. 231, Amer. Math. Soc., Providence, RI (1999) 73-85 MR1705576

[18] M Farber, V Turaev, Poincaré-Reidemeister metric, Euler structures, and torsion, J. Reine Angew. Math. 520 (2000) 195-225 MR1748274

[19] P B Gilkey, The eta invariant and secondary characteristic classes of locally flat bundles, from: "Algebraic and differential topology - global differential geometry", Teubner-Texte Math. 70, Teubner, Leipzig (1984) 49-87 MR792686

[20] G Grubb, R T Seeley, Weakly parametric pseudodifferential operators and AtiyahPatodi-Singer boundary problems, Invent. Math. 121 (1995) 481-529 MR1353307

[21] V Guillemin, A new proof of Weyl's formula on the asymptotic distribution of eigenvalues, Adv. in Math. 55 (1985) 131-160 MR772612

[22] R-T Huang, Refined analytic torsion and the eta-invariant, $\mathrm{PhD}$ thesis (in preparation)

[23] R-T Huang, Refined analytic torsion: comparison theorems and examples, Illinois J. Math. (to appear) arXiv:math.DG/0602231

[24] A S Markus, Introduction to the spectral theory of polynomial operator pencils, Translations of Mathematical Monographs 71, American Mathematical Society, Providence, RI (1988) Translated from the Russian by H H McFaden. Translation edited by B Silver. With an appendix by M V Keldysh.

[25] J Milnor, Whitehead torsion, Bull. Amer. Math. Soc. 72 (1966) 358-426 MR0196736

[26] L I Nicolaescu, The Reidemeister torsion of 3-manifolds, de Gruyter Studies in Mathematics 30, Walter de Gruyter \& Co., Berlin (2003) MR1968575

[27] R Ponge, Spectral asymmetry, zeta Functions and the noncommutative residue, Int. Math. J. (to appear) arXiv:math.DG/0310102

[28] D Quillen, Determinants of Cauchy-Riemann operators on Riemann surfaces, Funktsional. Anal. i Prilozhen. 19 (1985) 37-41, 96

[29] D B Ray, I M Singer, $R$-torsion and the Laplacian on Riemannian manifolds, Advances in Math. 7 (1971) 145-210 MR0295381 
[30] Y B Rudyak, On Thom spectra, orientability and cobordism, Springer Monographs in Mathematics, Springer, Berlin (1998) MR1627486 With a foreword by Haynes Miller

[31] R T Seeley, Complex powers of an elliptic operator, from: "Singular Integrals (Proc. Sympos. Pure Math., Chicago, Ill., 1966)”, Amer. Math. Soc., Providence, R.I. (1967) 288-307 MR0237943

[32] M A Shubin, Pseudodifferential operators and spectral theory, second edition, Springer, Berlin (2001) MR1852334 Translated from the 1978 Russian original by Stig I. Andersson

[33] I M Singer, Families of Dirac operators with applications to physics, Astérisque (1985) 323-340 MR837207The mathematical heritage of Élie Cartan (Lyon, 1984)

[34] G Su, W Zhang, A Cheeger-Mueller theorem for symmetric bilinear torsions arXiv: math.DG/0610577

[35] V G Turaev, Reidemeister torsion in knot theory, Uspekhi Mat. Nauk 41 (1986) 97-147, 240 MR832411

[36] V G Turaev, Euler structures, nonsingular vector fields, and Reidemeister-type torsions, Izv. Akad. Nauk SSSR Ser. Mat. 53 (1989) 607-643, 672 MR1013714

[37] V Turaev, Introduction to combinatorial torsions, Lectures in Mathematics ETH Zürich, Birkhäuser Verlag, Basel (2001) MR1809561Notes taken by Felix Schlenk

[38] C T C Wall, Determination of the cobordism ring, Ann. of Math. (2) 72 (1960) 292-311 MR0120654

[39] M Wodzicki, Local invariants of spectral asymmetry, Invent. Math. 75 (1984) 143-177 MR728144

[40] M Wodzicki, Noncommutative residue. I. Fundamentals, from: “ $K$-theory, arithmetic and geometry (Moscow, 1984-1986)”, Lecture Notes in Math. 1289, Springer, Berlin (1987) 320-399

[41] K P Wojciechowski, Heat equation and spectral geometry. Introduction for beginners, from: "Geometric methods for quantum field theory (Villa de Leyva, 1999)", World Sci. Publ., River Edge, NJ (2001) 238-292 MR1867735

Department of Mathematics, Northeastern University

Boston MA 02115, USA

Institut fur Mathematik, Universitat Zürich

Winterthurerstrasse 190, CH-8057 Zürich, Switzerland

maximbraverman@neu.edu, tk@math.unizh.ch

Proposed: Peter Teichner

Seconded: Wolfgang Lueck, Leonid Polterovich

Received: 29 March 2006

Accepted: 21 November 2006

Geometry 6 Topology, Volume 11 (2007) 